\title{
ON CONSTANT MEAN CURVATURE SURFACES WITH PERIODIC METRIC
}

\author{
J. Dorfmeister And G. HAAK
}

\begin{abstract}
We investigate CMC-surfaces with periodic metric in a dressing orbit of the cylinder. It is shown, that such surfaces are always of finite type. Using the periodicity conditions for the extended frame of a CMC-surface, we develop an alternative approach to the classification of CMC-tori given by Pinkall and Sterling.
\end{abstract}

\section{Introduction.}

In recent years two independent approaches to the construction of CMCimmersions in $\mathbb{R}^{3}$ were developed. One of them, using finite type solutions of the sinh-Gordon equation, leads to the classification of CMC-tori by Pinkall and Sterling [20].

While this approach allowed A. Bobenko [1] to describe CMC-tori explicitly in terms of theta functions, it turned out not to be easily adaptable to the more general situation of CMC-surfaces with umbilics.

The class of CMC-surfaces with umbilics contains the CMC-surfaces of higher genus, whose existence was proved by N. Kapouleas for arbitrary genus $[17,16]$. Therefore, it proved to be necessary to find a more general description of CMC-immersions.

Another approach to CMC-surfaces was developed by F. Pedit, H. Wu and one of the authors (J.D.). It is commonly called the DPW method. It gives a description of all immersed CMC-surfaces in $\mathbb{R}^{3}$ with or without umbilics. More specifically, it allows the construction of CMC-immersions from a meromorphic and a holomorphic function, for which reason it can be called a Weierstraß type representation of CMC-immersions. These functions, one of which is the coefficient of the Hopf differential of the surface to be constructed, are only subject to some simple restrictions [3].

The main problem with the DPW method as opposed to the Weierstraß representation for minimal surfaces is the fact, that in spite of the simplicity of the initial data, it is not easy to extract further geometric information out of these data. For one point, it turns out to be very hard to impose restrictions on the symmetries of the surface in $\mathbb{R}^{3}$ or on the Fuchsian or elementary group of the underlying Riemann surface. One of the cases where 
this would be necessary is the case of compact surfaces. In fact, it was not even clear how to reproduce the results of Pinkall, Sterling [20] and Bobenko [1] with the DPW method.

In this paper we will solve this problem and reproduce the classification of CMC-tori using extended frames without referring to the metric, i.e., the sinh-Gordon equation, explicitly. This result will be proved in a more general framework, which was developed in [4] by the authors for the investigation of CMC-surfaces with symmetries in $\mathbb{R}^{3}$. Here we will actually derive a classification of all finite type surfaces with periodic metric, i.e., surfaces whose metric is a finite type solution of the sinh-Gordon equation which is invariant under a group of translations in $\mathbb{C}$. This classification is of course also implicit in [20].

But first, let us define more precisely, what we mean by a CMC-surface with periodic metric. Here we follow [4].

1.2. Let $\Psi: \mathbb{C} \rightarrow \mathbb{R}^{3}$ be a conformal CMC-immersion of the complex plane into $\mathbb{R}^{3}$. We further set OAff $\left(\mathbb{R}^{3}\right)$ to be the group of proper (i.e., orientation preserving) Euclidean motions in $\mathbb{R}^{3}$.

Definition. Let $\Psi: \mathbb{C} \rightarrow \mathbb{R}^{3}$ be defined as above. Then we define the following subset $\operatorname{Sym}(\Psi)$ of $\mathbb{C}$ : A complex constant $q$ is in $\operatorname{Sym}(\Psi)$, iff there exists a proper Euclidean motion $T_{q} \in \operatorname{OAff}\left(\mathbb{R}^{3}\right)$, s.t.

$$
\Psi(z+q)=T_{q}(\Psi(z)) \quad \text { for all } z \in \mathbb{C} .
$$

If $\operatorname{Sym}(\Psi) \neq\{0\}$, then we say that $\Psi$ is a surface with periodic metric.

We further define the subset $\operatorname{Per}(\Psi)$ of $\operatorname{Sym}(\Psi)$ by

$$
\operatorname{Per}(\Psi)=\{q \in \mathbb{C} \mid \Psi(z+q)=\Psi(z), \text { for all } z \in \mathbb{C}\} .
$$

If $\operatorname{Per}(\Psi) \neq\{0\}$, then we say that $\Psi$ is a periodic surface.

From time to time we will also identify the elements of $\operatorname{Sym}(\Psi)$ and $\operatorname{Per}(\Psi)$ with the corresponding translations, i.e., we will identify $\operatorname{Sym}(\Psi)$ and $\operatorname{Per}(\Psi)$ with subgroups of Aut $\mathbb{C}$, the set of biholomorphic automorphisms of $\mathbb{C}$.

For the investigation of translational symmetries, it is of course enough to consider only those Riemannian surfaces whose universal cover is the whole complex plane. Therefore, we will not introduce the more complex notation of [4]. We collect here some facts about the groups $\operatorname{Sym}(\Psi)$ and $\operatorname{Per}(\Psi)$ which follow from results in [4]. For details and the definition of the associated family in this context see [4, Sect. 2.5].

Theorem. Let $(\mathbb{C}, \Psi)$ be a conformal CMC-immersion. Then the following holds:

(1) If $\operatorname{Sym}(\Psi)$ is nondiscrete, then $\Psi(\mathbb{C})$ is in the associated family of a Delaunay surface. 
(2) If $\operatorname{Sym}(\Psi)$ is discrete and has a nonzero element, then its elements form a lattice in $\mathbb{C}$ with one or two generators.

(3) $\operatorname{Per}(\Psi)$ is always discrete and, if nontrivial, forms a lattice in $\mathbb{C}$ with one or two generators.

(4) If $\operatorname{Per}(\Psi) \neq\{0\}$, then there exists a Riemannian surface $M$ with universal cover $\pi: \mathbb{C} \rightarrow M$ and a CMC-immersion $\Phi: M \rightarrow \mathbb{R}^{3}$, s.t. $\Gamma=\operatorname{Per}(\Psi)$ is the elementary group of $M$ and $\Psi=\Phi \circ \pi$.

(5) In particular, if $\operatorname{Per}(\Psi)$ forms a lattice with two generators, then $\Psi(\mathbb{C})$ is a CMC-torus.

The paper starts in Section 2 with a short description of the DPW method for later comparison with other formulations. We will also give the transformation properties of an extended frame under a translation in $\operatorname{Sym}(\Psi)$ as they were derived in [4, Sec. 3.4]. In this section we will work with loop groups on arbitrary circles $C_{r}, 0<r<1$, which allows for a more general definition of the dressing action (see also $[2,5]$ ).

In Chapter 3 we consider surfaces obtained by $r$-dressing $[7,2]$ from the standard cylinder:

$$
h_{+}(\lambda) e^{\left(\lambda^{-1} z-\lambda \bar{z}\right) A}=F(z, \bar{z}, \lambda) p_{+}(z, \bar{z}, \lambda) .
$$

Here $A=\left(\begin{array}{ll}0 & 1 \\ 1 & 0\end{array}\right)$ and $F$ is the (unitary) frame of the surface, while $p_{+}$and $h_{+}$, in a Fourier expansion, does not ontain any negative powers of $\lambda$. By a result of [7] (see also [2]) all CMC-tori can be obtained this way. The main result of this chapter considers translations $z \mapsto z+q$ and gives necessary and sufficient conditions on $h_{+}$such that the frame $F$ at $z+q$ is a $z$-independent rotation of $F$ at $z$ for all $z \in \mathbb{C}$ (Theorem 3.7, Theorem 3.8). This is equivalent with the metric of the surface being periodic with period $q$. As a consequence, in Theorem 3.9 we give necessary and sufficient conditions, that a CMC-surface, obtained by $r$-dressing of the standard cylinder by $h_{+}$, admits the translation $z \mapsto z+q$ in its fundamental group. This describes all topological cylinders obtained by $r$-dressing the standard cylinder.

The results of Chapter 3 are phrased in terms of certain even rational functions $a^{2}\left(\lambda^{2}\right), b^{2}\left(\lambda^{2}\right), c^{2}\left(\lambda^{2}\right)$ and meromorphic functions $\alpha, \beta^{2}$ on $\mathbb{C} \backslash\{0\}$. In Chapter 4 we consider the hyperelliptic surface $\mathcal{C}$ with branchpoints exactly at the zeroes and poles of odd order of $a^{2}$ and a natural two-fold covering $\mathcal{C}^{\prime}$ of $\mathcal{C}$. It turns out that all functions involved in the $r$-dressing, and a priori only defined on the disk of radius $r$ around $\lambda=0$, can be extended holomorphically to the hyperelliptic surface $\mathcal{C}$ or at least to the hyperelliptic surface $\mathcal{C}^{\prime}$. As an application of this extension we show (Theorem 4.9), that every CMC-surface with periodic metric in the $r$-dressing orbit of the 
standard cylinder is of finite type (for a definition of "finite type" in this framework see $[2,7,20]$ ).

In Chapter 5 we start from a hyperelliptic surface $\mathcal{C}$ and define $\alpha, \beta^{2}, a^{2}, b^{2}$, $c^{2}$ in a natural way, thus producing $a^{2}$ with only simple poles. In this case, which is generic for CMC-tori, we reproduce the dressing matrix and thus are able to describe (generically) all CMC-tori in the $r$-dressing orbit of the cylinder (Section 5.8). If $g$ is the genus of $\mathcal{C}$, then our construction yields for every CMC-torus in the $r$-dressing orbit of the cylinder naturally a $g-1$ parameter family of tori. This fits well to the purely algebro-geometric construction of such families of tori as given in [1, 9, 15].

It would be interesting to expand our construction in Chapter 5 to the "non-generic" case, i.e., to the case where $a^{2}$ does not only have simple poles and zeroes of odd order.

\section{The DPW method.}

Let us recall for the reader's convenience the cornerstones of the DPW method. For a more detailed reference the reader should consult $[6,5]$ and the appendix of [3]. In this section we will follow the conventions of [3]. By a CMC-immersion we will always understand a conformal immersion of constant mean curvature. For a justification of this restriction, see [4, Section 2].

2.1. Let $\Psi: \mathcal{D} \rightarrow \mathbb{R}^{3}$ be a CMC-immersion. Here, $\mathcal{D} \subset \mathbb{C}$ is the open unit disk or the whole complex plane. We take $\Psi$ as a conformal chart and the metric to be $\mathrm{d} s^{2}=e^{u}\left(\mathrm{~d} x^{2}+\mathrm{d} y^{2}\right), u: \mathcal{D} \rightarrow \mathbb{R}$. Then

$$
\mathcal{U}=\left(e^{-\frac{u}{2}} \Psi_{x}, e^{-\frac{u}{2}} \Psi_{y}, N\right): \mathcal{D} \longrightarrow \mathbf{S O}(3)
$$

is an orthonormal frame which we normalize by

$$
\mathcal{U}(x=0, y=0)=I .
$$

In complex coordinates $z=x+i y, \bar{z}=x-i y$, we have

$$
\left\langle\Psi_{z}, \Psi_{z}\right\rangle=\left\langle\Psi_{\bar{z}}, \Psi_{\bar{z}}\right\rangle=0, \quad\left\langle\Psi_{z}, \Psi_{\bar{z}}\right\rangle=\frac{1}{2} e^{u}
$$

Using the definitions

$$
E=\left\langle\Psi_{z z}, N\right\rangle, \quad H=2 e^{-u}\left\langle\Psi_{z \bar{z}}, N\right\rangle
$$

we get for the second fundamental form

$$
I I=\frac{1}{2}\left(\begin{array}{cc}
(E+\bar{E})+H e^{u} & i(E-\bar{E}) \\
i(E-\bar{E}) & -(E+\bar{E})+H e^{u}
\end{array}\right) .
$$


Thus, $H$ is the mean curvature and therefore constant. The Gauß-Codazzi equations take the form

$$
\begin{gathered}
u_{z \bar{z}}+\frac{1}{2} e^{u} H^{2}-2 e^{-u}|E|^{2}=0, \\
E_{\bar{z}}=\frac{1}{2} e^{u} H_{z}=0,
\end{gathered}
$$

from the second of which it follows, that $E \mathrm{~d} z^{2}$ is a holomorphic differential, the Hopf differential. For $|E|=1$ and $H=2$ the first equation becomes the sinh-Gordon equation.

By using the spinor representation $J: \mathbf{r} \mapsto-\frac{i}{2} \mathbf{r} \sigma$, where $\sigma$ is the vector, whose components are the Pauli matrices, we identify vectors in $\mathbb{R}^{3}$ with matrices in $\mathbf{s u}(2)$. This induces an identification of orthonormal frames with matrices in $\mathbf{S U}(2)$ (see [3]) which is unique up to the multiplication with $-I$ in $\mathbf{S U}(2)$. If we identify the frame $\mathcal{U}$ in this way with a map

$$
P: \mathcal{D} \rightarrow \mathbf{S U}(2),
$$

then the initial condition (2.1.2) is compatible with

$$
P(0)=I,
$$

which fixes the lift $P$ uniquely. If we define

$$
\begin{aligned}
& U=P^{-1} P_{z}=\left(\begin{array}{cc}
-\frac{1}{4} u_{z} & E e^{-\frac{u}{2}} \\
-\frac{1}{2} e^{\frac{u}{2}} H & \frac{1}{4} u_{z}
\end{array}\right), \\
& V=P^{-1} P_{\bar{z}}=\left(\begin{array}{cc}
\frac{1}{4} u_{\bar{z}} & \frac{1}{2} H e^{\frac{u}{2}} \\
-\bar{E} e^{-\frac{u}{2}} & -\frac{1}{4} u_{\bar{z}}
\end{array}\right),
\end{aligned}
$$

then the integrability condition

$$
U_{\bar{z}}-V_{z}-[U, V]=0
$$

is equivalent to the Gauß-Codazzi equations (2.1.6) and (2.1.7).

By the substitution $E \rightarrow \lambda^{-2} E, \lambda \in S^{1}$, which doesn't change the GaußCodazzi equations, we introduce the so called spectral parameter $\lambda$. After a simple re-gauging of the frames (for details see the appendix of [3]) we get the following form of the now $\lambda$-dependent matrices $U(\lambda)$ and $V(\lambda)$ :

$$
\begin{aligned}
& U(\lambda)=\left(\begin{array}{cc}
\frac{1}{4} u_{z} & -\frac{1}{2} \lambda^{-1} H e^{\frac{u}{2}} \\
\lambda^{-1} E e^{-\frac{u}{2}} & -\frac{1}{4} u_{z}
\end{array}\right), \\
& V(\lambda)=\left(\begin{array}{cc}
-\frac{1}{4} u_{\bar{z}} & -\lambda \bar{E} e^{-\frac{u}{2}} \\
\frac{1}{2} \lambda H e^{\frac{u}{2}} & \frac{1}{4} u_{\bar{z}}
\end{array}\right) .
\end{aligned}
$$


By integration, i.e., solving

$$
\begin{aligned}
& F^{-1} F_{z}=U, \\
& F^{-1} F_{\bar{z}}=V,
\end{aligned}
$$

using the initial condition

$$
F(0, \lambda)=I, \quad \text { for all } \lambda \in S^{1},
$$

we get a $\lambda$-dependent frame $F(z, \bar{z}, \lambda)$, which coincides at $\lambda=1$ with the old frame $P(z, \bar{z})$. We call this map from $\mathcal{D} \times S^{1}$ to $\mathbf{S U}(2)$ the extended frame of the immersion $\Psi$. It should be noted, that by Eq. (2.1.13) and (2.1.14) the differential of $F$ has the form

$$
\begin{aligned}
\alpha(z, \bar{z}, \lambda) & =\lambda^{-1} \alpha_{-1}(z, \bar{z}) \mathrm{d} z+\alpha_{0}(z, \bar{z})+\lambda \alpha_{1}(z, \bar{z}) \mathrm{d} \bar{z} \\
\alpha_{1}(z, \bar{z}) & =-\bar{\alpha}_{-1}(z, \bar{z})
\end{aligned}
$$

This map is now interpreted as taking values in a certain loop algebra $\Lambda_{r} \mathbf{s u}(2)_{\sigma}$.

2.2. For each real constant $r, 0<r<1$, let $\Lambda_{r} \mathbf{S L}(2, \mathbb{C})_{\sigma}$ denote the group of smooth maps $g(\lambda)$ from $C_{r}$, the circle of radius $r$, to $\mathbf{S L}(2, \mathbb{C})$, which satisfy the twisting condition

$$
g(-\lambda)=\sigma(g(\lambda))
$$

where $\sigma: \mathbf{S L}(2, \mathbb{C}) \rightarrow \mathbf{S L}(2, \mathbb{C})$ is the group automorphism of order 2 , which is given by conjugation with the Pauli matrix

$$
\sigma_{3}=\left(\begin{array}{cc}
1 & 0 \\
0 & -1
\end{array}\right)
$$

The Lie algebras of these groups, which we denote by $\Lambda_{r} \mathbf{s l}(2, \mathbb{C})_{\sigma}$, consist of maps $x: C_{r} \rightarrow \mathbf{s l}(2, \mathbb{C})$, which satisfy a similar twisting condition as the group elements

$$
x(-\lambda)=\sigma_{3} x(\lambda) \sigma_{3}
$$

In order to make these loop groups complex Banach Lie groups, we equip them, as in [6], with some $H^{s}$-topology for $s>\frac{1}{2}$. Elements of these twisted loop groups are matrices with off-diagonal entries which are odd functions, and diagonal entries which are even functions in the parameter $\lambda$. All entries are in the Banach algebra $\mathcal{A}_{r}$ of $H^{s}$-smooth functions on $C_{r}$. 
Furthermore, we will use the following subgroups of $\Lambda_{r} \mathbf{S L}(2, \mathbb{C})_{\sigma}$ : Let B be a subgroup of $\mathbf{S L}(2, \mathbb{C})$ and $\Lambda_{r, B}^{+} \mathbf{S L}(2, \mathbb{C})_{\sigma}$ be the group of maps in $\Lambda_{r} \mathbf{S L}(2, \mathbb{C})_{\sigma}$, which can be extended to holomorphic maps on

$$
I^{(r)}=\{\lambda \in \mathbb{C} ;|\lambda|<r\},
$$

the interior of the circle $C_{r}$, and take values in $\mathbf{B}$ at $\lambda=0$. Analogously, let $\Lambda_{r, B}^{-} \mathbf{S L}(2, \mathbb{C})_{\sigma}$ be the group of maps in $\Lambda_{r} \mathbf{S L}(2, \mathbb{C})_{\sigma}$, which can be extended to the exterior

$$
E^{(r)}=\left\{\lambda \in \mathbb{C P}_{1} ;|\lambda|>r\right\}
$$

of $C_{r}$ and take values in $\mathbf{B}$ at $\lambda=\infty$. If $\mathbf{B}=\{I\}$ (based loops) we write the subscript $*$ instead of $\mathbf{B}$, if $\mathbf{B}=\mathbf{S L}(2, \mathbb{C})$ we omit the subscript for $\Lambda$ entirely.

Also, by an abuse of notation, we will denote by $\Lambda_{r} \mathbf{S U}(2)_{\sigma}$ the subgroup of maps in $\Lambda_{r} \mathbf{S L}(2, \mathbb{C})_{\sigma}$, which can be extended holomorphically to the open annulus

$$
A^{(r)}=\left\{\lambda \in \mathbb{C} ; r<|\lambda|<\frac{1}{r}\right\}
$$

and take values in $\mathbf{S U}(2)$ on the unit circle.

Corresponding to these subgroups, we analogously define Lie subalgebras of $\Lambda_{r} \mathbf{s l}(2, \mathbb{C})_{\sigma}$.

We quote the following results from [18] and [6]:

(i) For each solvable subgroup $\mathbf{B}$ of $\mathbf{S L}(2, \mathbb{C})$, which satisfies $\mathbf{S U}(2) \cdot \mathbf{B}=$ $\mathbf{S L}(2, \mathbb{C})$ and $\mathbf{S U}(2) \cap \mathbf{B}=\{I\}$, multiplication

$$
\Lambda_{r} \mathbf{S U}(2)_{\sigma} \times \Lambda_{r, B}^{+} \mathbf{S L}(2, \mathbb{C})_{\sigma} \longrightarrow \Lambda_{r} \mathbf{S L}(2, \mathbb{C})_{\sigma}
$$

is a diffeomorphism onto. The associated splitting

$$
g=F g_{+}
$$

of an element $g$ of $\Lambda_{r} \mathbf{S L}(2, \mathbb{C})_{\sigma}$, s.t. $F \in \Lambda_{r} \mathbf{S U}(2)_{\sigma}$ and $g_{+} \in \Lambda_{r, B}^{+} \mathbf{S L}(2, \mathbb{C})_{\sigma}$ will be called Iwasawa decomposition. In the following, we will fix the group $\mathbf{B}$ as the group of upper triangular $2 \times 2$-matrices with real positive entries on the diagonal.

(ii) Multiplication

$$
\Lambda_{r, *}^{-} \mathbf{S L}(2, \mathbb{C})_{\sigma} \times \Lambda_{r}^{+} \mathbf{S L}(2, \mathbb{C})_{\sigma} \longrightarrow \Lambda_{r} \mathbf{S L}(2, \mathbb{C})_{\sigma}
$$


is a diffeomorphism onto the open and dense subset $\Lambda_{r, *}^{-} \mathbf{S L}(2, \mathbb{C})_{\sigma}$. $\Lambda_{r}^{+} \mathbf{S L}(2, \mathbb{C})_{\sigma}$ of $\Lambda_{r} \mathbf{S L}(2, \mathbb{C})_{\sigma}$, called the "big cell" [21]. The associated splitting

$$
g=g_{-} g_{+}
$$

of an element $g$ of the big cell, where $g_{-} \in \Lambda_{r, *}^{-} \mathbf{S L}(2, \mathbb{C})_{\sigma}$ and $g_{+} \in$ $\Lambda_{r}^{+} \mathbf{S L}(2, \mathbb{C})_{\sigma}$, will be called Birkhoff factorization.

Clearly, for arbitrary $0<r<1$, the matrices $U(\lambda)$ and $V(\lambda)$ defined above take values in $\Lambda_{r} \mathbf{s u}(2)_{\sigma}$, and so does $\alpha$.

We also get:

Lemma. The extended frame $F(z, \bar{z}, \lambda)$ of a CMC-immersion can be extended holomorphically in $\lambda$ to $\mathbb{C}^{*}=\mathbb{C} \backslash\{0\}$.

Proof. For $n \in \mathbb{N}$ define $K_{n}=\left\{\lambda \in \mathbb{C} ; \frac{1}{n} \leq|\lambda| \leq n\right\}$. Then $\left\{K_{n}\right\}_{n \in \mathbb{N}}$ is a sequence of compact subsets of $\mathbb{C}^{*}$, which exhaust $\mathbb{C}^{*}$. For each $n \in \mathbb{N}$, $\alpha(z, \bar{z}, \lambda)$, defined by (2.1.18), takes values in the Banach algebra $C\left(K_{n}\right)$ of bounded functions on $K_{n}$, which are holomorphic in the interior of $K_{n}$. Since also the initial condition (2.1.17) for $F$ is in $C\left(K_{n}\right)$, we get that for each $n \in \mathbb{N}$, the solution $F(z, \bar{z}, \lambda)$ of the differential equation $F^{-1} \mathrm{~d} F=\alpha$ takes values in $C\left(K_{n}\right)$. Therefore, $F$ can be continued holomorphically in the $\lambda$-plane to the open set

$$
\bigcup_{n \in \mathbb{N}} K_{n}=\mathbb{C}^{*}
$$

Using the initial condition $F(0, \lambda)=I$ we thus see, that, for each radius $r$, the extended frame $F$ is, by restriction to $C_{r}$, a map from $\mathcal{D}$ to $\Lambda_{r} \mathbf{S U}(2)_{\sigma}$. We will use this fact to identify the extended frames with elements of $\Lambda_{r} \mathbf{S U}(2)_{\sigma}$.

If $F(z, \bar{z}, \lambda)$ is the extended frame of a CMC-immersion $\Psi$, then Sym's formula

$$
(2.2 .11) J(\Psi(z, \bar{z}, \lambda))=-\frac{1}{2 H}\left(\frac{\partial F}{\partial \theta} F^{-1}+\frac{i}{2} F \sigma_{3} F^{-1}\right), \quad \lambda=e^{i \theta},
$$

gives a one parameter family of CMC-immersions in the spinor representation. The original CMC-immersion $\Psi$ is reproduced by setting $\lambda=1$. The whole family $\Psi_{\lambda}$ of CMC-immersions is usually called the associated family of $\Psi$. 
2.3. The following result was proved in [6]:

Theorem. Let $F: \mathcal{D} \rightarrow \Lambda_{r} \boldsymbol{S U}(2)_{\sigma}, \mathcal{D} \subset \mathbb{C}$, simply connected, and let $\Psi_{\lambda}: \mathcal{D} \rightarrow \mathbb{R}^{3}, \lambda \in S^{1}$, be defined by Sym's formula (2.2.11). Then the following are equivalent:

(1) For each $\lambda \in S^{1}$ the map $\Psi_{\lambda}: \mathcal{D} \rightarrow \mathbb{R}^{3}$ is a CMC-immersion,

(2) The $\Lambda_{r} \boldsymbol{s u}(2)_{\sigma}$-valued 1-form $\alpha:=F^{-1} \mathrm{~d} F$ is of the form (2.1.18).

The construction of such extended frames is the goal of the DPW method.

To get the so called meromorphic potential from the extended frame we utilize the Birkhoff splitting: We define the map $g_{-}: \mathcal{D} \rightarrow \Lambda_{r, *}^{-} \mathbf{S L}(2, \mathbb{C})_{\sigma}$ by

$$
F(z, \bar{z}, \lambda)=g_{-}(z, \bar{z}, \lambda) g_{+}(z, \bar{z}, \lambda)
$$

where $g_{+}$takes values in $\Lambda_{r}^{+} \mathbf{S L}(2, \mathbb{C})_{\sigma}$.

The derivative

$$
\xi=g_{-}^{-1} \mathrm{~d} g_{-}
$$

is a one form taking values in $\Lambda_{r, *}^{-} \mathbf{s l}(2, \mathbb{C})_{\sigma}$. By a simple calculation using the definitions (2.1.13), (2.1.14) of $U(\lambda)$ and $V(\lambda)$, it is easily shown, that $\xi(z, \lambda)$ is of the form

$$
\xi(z, \lambda)=\lambda^{-1}\left(\begin{array}{cc}
0 & f(z) \\
\frac{E(z)}{f(z)} & 0
\end{array}\right) \mathrm{d} z,
$$

where $E(z) \mathrm{d} z^{2}$ is the Hopf differential and $f(z)$ is a scalar meromorphic function. The meromorphic potential has poles in the set $S \subset \mathcal{D}$ which consists of those points $z \in \mathcal{D}$, for which $F(z, \bar{z}, \lambda)$ is not in the big cell. As a function in $\lambda$ it can be extended holomorphically to $\mathbb{C} \mathrm{P}_{1} \backslash\{0\}$.

2.4. Conversely, given a meromorphic potential of the form (2.3.3) one can use the Iwasawa decomposition

$$
g_{-}(z, \bar{z}, \lambda)=F(z, \bar{z}, \lambda) g_{+}(z, \bar{z}, \lambda)^{-1}
$$

at every point in $\mathcal{D}$ to get back a map $F$ from $\mathcal{D}$ to $\Lambda_{r} \mathbf{S U}(2)_{\sigma}$.

A simple calculation then shows, that the 1-form $\alpha:=F^{-1} \mathrm{~d} F$ is of the form (2.1.18). Therefore, $F(z, \bar{z}, \lambda)$ defined by Eq. (2.4.1) is indeed an extended frame.

It should also be noted, that this construction does not depend on the chosen radius $r$ : Since the meromorphic potential, and therefore also $g_{-}(z, \lambda)$ can be continued holomorphically (in $\lambda$ ) to $\mathbb{C} \mathrm{P}_{1} \backslash\{0\}$, the extended frame $F(z, \bar{z}, \lambda)$ can be continued holomorphically to $\mathbb{C}^{*}=\mathbb{C} \backslash\{0\}$. Therefore, the 
Iwasawa decomposition of $g_{-}$at $C_{r}$ gives always the same $F$ restricted to the respective circle $C_{r}$. This shows that there is only one DPW-method, not a whole family of " $r$-DPW methods".

2.5. Another construction of interest here is the dressing method. It allows to construct new CMC-immersions from old ones.

In the loop group formalism the dressing method has a very simple formulation.

Given an extended frame $F_{0}$, we take an arbitrary radius $0<r<1$ and an element $h_{+}$of $\Lambda_{r}^{+} \mathbf{S L}(2, \mathbb{C})_{\sigma}$ and compute the Iwasawa splitting of $h_{+} F_{0}$ at every point in $\mathcal{D}$,

$$
h_{+}(\lambda) F_{0}(z, \bar{z}, \lambda)=F(z, \bar{z}, \lambda) p_{+}(z, \bar{z}, \lambda),
$$

where $F$ is a new map from $\mathcal{D}$ to $\Lambda_{r} \mathbf{S U}(2)_{\sigma}$ and $p_{+}$is a map from $\mathcal{D}$ to $\Lambda_{r}^{+} \mathbf{S L}(2, \mathbb{C})_{\sigma}$. Here we choose $p_{+}$such that $F$ satisfies $F(0, \lambda)=I$ for all $\lambda \in S^{1}$. Again, an elementary calculation shows, that $F$ is the extended frame of a (new) CMC-immersion.

$$
\text { If } \xi_{0}=\lambda^{-1}\left(\begin{array}{cc}
0 & f_{0} \\
\frac{E_{0}}{f_{0}} & 0
\end{array}\right) \mathrm{d} z \text { is the meromorphic potential for } F_{0} \text {, then the new }
$$
meromorphic potential $\xi$ for $F$ is given by

$$
\xi=\lambda^{-1}\left(\begin{array}{cc}
0 & f \\
\frac{E}{f} & 0
\end{array}\right) \mathrm{d} z=p_{+}^{-1} \xi_{0} p_{+}+p_{+}^{-1} \mathrm{~d} p_{+} .
$$

From this it follows, that $E=E_{0}$, i.e., the Hopf differential is invariant under dressing.

As in the case of the DPW method it can be easily seen, that for given $h_{+}$this construction does not depend on the chosen radius $r$, as long as $h_{+}$is defined on $C_{r}$ and can be extended holomorphically to $I^{(r)}$. Thus, if we denote the dressing orbit of $F^{0}$ w.r.t. $r$ by $\mathcal{O}_{r}\left(F^{0}\right)$, we have a canonical inclusion

$$
\mathcal{O}_{r}\left(F^{0}\right) \subset \mathcal{O}_{r^{\prime}}\left(F^{0}\right) \quad \text { for } 0<r^{\prime}<r<1 .
$$

2.6. Finally, we want to state the transformation properties of an extended frame under a translation in $\operatorname{Sym}(\Psi)$ as they were derived in [4], Section 3.1-3.3. From here on we will set $\mathcal{D}=\mathbb{C}$. For notational convenience we will also from now on omit the $\bar{z}$-dependency.

Theorem. Let $\Psi(z)$ be a CMC-immersion with extended frame $F(z, \lambda)$ and associated family $\Psi_{\lambda}(z), \Psi(z)=\Psi_{1}(z)$.

If $q \in \operatorname{Sym}(\Psi)$ then $F(z, \lambda)$ transforms under the translation by $q$ as

$$
F(z+q, \lambda)=\chi(q, \lambda) F(z, \lambda)
$$


where $\chi(q, \lambda)$ can be extended holomorphically to $\mathbb{C}^{*}$. For arbitrary $0<$ $r<1$, restriction to $C_{r}$ gives a group homomorphism $\chi(\cdot, \lambda): \operatorname{Sym}(\Psi) \rightarrow$ $\Lambda_{r} \boldsymbol{S U}(2)_{\sigma}$, i.e.

$$
\chi\left(q_{1}+q_{2}, \lambda\right)=\chi\left(q_{1}, \lambda\right) \chi\left(q_{2}, \lambda\right), \quad q_{1}, q_{2} \in \operatorname{Sym}(\Psi) .
$$

Proof. By Remark 1.2, Eqs. (2.6.1) and (2.6.2) follow for $\lambda \in S^{1}$ immediately from [4, Lemma 3.1, Theorem 3.3]. Since, by (2.1.17), $\chi(q, \lambda)=F(q, \lambda)$ we get using Lemma 2.2 , that $\chi(q, \lambda)$ can be extended holomorphically to $\mathbb{C}^{*}$. Therefore, restriction to $C_{r}, 0<r<1$, gives $\chi(q, \lambda) \in \Lambda_{r} \mathbf{S U}(2)_{\sigma}$ and the rest follows by uniqueness of analytic continuation.

As we did already for extended frames, we can, for arbitrary radius $0<r<1$, view $\chi(q, \lambda)$ as an element of $\Lambda_{r} \mathbf{S U}(2)_{\sigma}$ by restricting it to the circle $C_{r}$.

2.7. Using Sym's formula (2.2.11), we can also easily compute the proper Euclidean motion $T_{q}$ in Eq. (1.2.1) from the map $\chi$ :

$$
\begin{aligned}
J(\Psi(z+q)) & \\
=- & \frac{1}{2 H}\left(\left.\frac{\partial \chi(q, \lambda)}{\partial \theta}\right|_{\theta=0} \cdot \chi(q, 1)^{-1}+\left.\chi \frac{\partial F(z, \lambda)}{\partial \theta}\right|_{\theta=0} \cdot F(z, 1)^{-1} \chi(q, 1)^{-1}\right. \\
& \left.\quad-\frac{i}{2} \chi(q, 1) F(z, 1) \sigma_{3} F(z, 1)^{-1} \chi(q, 1)^{-1}\right) \\
= & \chi(q, 1) J(\Psi(z)) \chi(q, 1)^{-1}-\left.\frac{1}{2 H} \frac{\partial \chi(q, \lambda)}{\partial \theta}\right|_{\theta=0} \cdot \chi(q, 1)^{-1}, \lambda=e^{i \theta} .
\end{aligned}
$$

Thus, $T_{q}$ is given by

$$
J\left(T_{q}(z)\right)=J\left(R_{q} \cdot z+t_{q}\right)
$$

where the rotation $R_{q}$ is in the spinor representation given by

$$
J\left(R_{q} . z\right)=\chi(q, 1) J(z) \chi(q, 1)^{-1}
$$

and the vector $t_{q} \in \mathbb{R}^{3}$ is given by

$$
J\left(t_{q}\right)=-\left.\frac{1}{2 H} \frac{\partial \chi(q, \lambda)}{\partial \theta}\right|_{\theta=0} \cdot \chi(q, 1)^{-1} .
$$

We get the following 
Theorem. Let $\Psi: \mathbb{C} \rightarrow \mathbb{R}^{3}$ be a CMC-immersion and let $q \in \operatorname{Sym}(\Psi)$.

Define, for $0<r<1, \chi(q, \lambda) \in \Lambda_{r} \boldsymbol{S} \boldsymbol{U}(2)_{\sigma}$ as above, then $q \in \operatorname{Per}(\Psi)$ iff

$$
\chi(q, 1)= \pm I \quad \text { and }\left.\quad \frac{\partial \chi\left(q, \lambda=e^{i \theta}\right)}{\partial \theta}\right|_{\theta=0}=0 .
$$

Proof. Follows from Eq. (2.7.1).

From (2.7.1) we immediately get the:

Corollary. Let $\Psi: \mathbb{C} \rightarrow \mathbb{R}^{3}, F(z, \lambda)$ and $\Psi_{\lambda}, \lambda \in S^{1}$ be as in Theorem 2.6. If $F(z, \lambda)$ transforms under the translation by $q \in \mathbb{C}^{*}$ as in (2.6.1), then $q \in \operatorname{Sym}\left(\Psi_{\lambda}\right)$ for all $\lambda \in S^{1}$.

Remark. It actually follows from [4, Prop. 3.4], that the group $\operatorname{Sym}\left(\Psi_{\lambda}\right)$ of translational symmetries is the same for all elements of the associated family. The group $\operatorname{Per}\left(\Psi_{\lambda}\right)$, however, depends crucially on $\lambda$ as we will see in Section 3.9.

2.8. For later use we will introduce the following antiholomorphic involution:

$$
\tau(\lambda)=\bar{\lambda}^{-1}
$$

Geometrically speaking, $\tau$ is the reflection at the unit circle. For a map $g(\lambda)$ from a subset of $\mathbb{C} \mathrm{P}_{1}$ to $\mathbf{S L}(2, \mathbb{C})$ we define

$$
g^{*}(\lambda)=\overline{g(\tau(\lambda))}^{\top}
$$

Thus, if $F \in \Lambda_{r} \mathbf{S L}(2, \mathbb{C})_{\sigma}$ is defined and holomorphic on $A^{(r)}$, then $F \in$ $\Lambda_{r} \mathbf{S U}(2)_{\sigma}$ is equivalent to

$$
F^{*}=F^{-1} \text {. }
$$

For a scalar function $f(\lambda)$ we set

$$
f^{*}(\lambda)=\overline{f(\tau(\lambda))}
$$

If $f$ is defined and holomorphic on a $\tau$-invariant neighbourhood of $S^{1}$, then $f$ is real on $S^{1}$ iff $f^{*}=f$. 


\section{Translational symmetries.}

In this chapter we will give necessary and sufficient conditions for a surface to be invariant under a given translation $z \mapsto z+q, q \in \mathbb{C}^{*}$. The conditions will be formulated in terms of certain functions that will be introduced in the next sections.

The calculations here are similar to those in [4, Section 3.7], with the exception, that we use here the $r$-loop formalism recalled in the previous section from [7]. In [4] these calculations were used to prove, that there are no translationally symmetric surfaces in the standard dressing orbit of the cylinder. However, by the result of [7, Corollary 5.3] (see also [2]), every CMC-torus is contained in the $r$-dressing orbit of the cylinder.

3.1. Let $F(z, \lambda)$ be defined by the $r$-dressing action of $h_{+} \in \Lambda_{r}^{+} \mathbf{S L}(2, \mathbb{C})_{\sigma}$ on the extended frame of the (standard) cylinder, i.e.,

$$
\begin{aligned}
& h_{+}(\lambda) e^{\left(\lambda^{-1} z-\lambda \bar{z}\right) A}=F(z, \lambda) \cdot p_{+}(z, \lambda), \\
& p_{+}(z, \lambda) \in \Lambda_{r}^{+} \mathbf{S L}(2, \mathbb{C})_{\sigma}, \quad A=\left(\begin{array}{ll}
0 & 1 \\
1 & 0
\end{array}\right) .
\end{aligned}
$$

We note that $e^{\left(\lambda^{-1} z-\lambda \bar{z}\right) A}$ is the extended frame associated with the meromorphic potential

$$
\xi=\lambda^{-1}\left(\begin{array}{ll}
0 & 1 \\
1 & 0
\end{array}\right) \mathrm{d} z
$$

and describes the (standard) cylinder. We also note that all functions of $z$ are defined on $\mathbb{C}$.

The frame $F(z, \lambda)$ transforms under the translation $z \mapsto z+q, q \in \mathbb{C}^{*}$, as

$$
F(z+q, \lambda)=Q(q, \lambda) F(z, \lambda) r_{+}(q, z, \lambda)
$$

where

$$
Q(q, \lambda)=h_{+} e^{\left(\lambda^{-1} q-\lambda \bar{q}\right) A} h_{+}^{-1}
$$

and

$$
r_{+}(q, z, \lambda)=p_{+}(z, \lambda) p_{+}(z+q, \lambda)^{-1} .
$$

3.2. By Theorem 2.6 and Corollary 2.7, we have that $q \in \operatorname{Sym}(\Psi)$, iff

$$
Q(q, \lambda) F(z, \lambda) r_{+}(q, z, \lambda)=\chi(q, \lambda) F(z, \lambda),
$$


where

$$
\chi(q, \lambda)=F(q, \lambda)
$$

is unitary on $S^{1}$. From Section 2.8 we thus know

$$
\chi^{*}(q, \lambda)=\chi(q, \lambda)^{-1} \quad \text { for } \lambda \in \mathbb{C}^{*} .
$$

We will derive further conditions on the matrix function $\chi$.

The initial condition (2.1.17) together with Eq. (3.2.1) implies

$$
\chi=Q \cdot R_{+},
$$

with

$$
R_{+}(q, \lambda)=r_{+}(q, 0, \lambda) \in \Lambda_{r}^{+} \mathbf{S L}(2, \mathbb{C})_{\sigma} .
$$

Thus, $F(z, \lambda)$ is invariant under the $r$-dressing transformation with $R_{+}$,

$$
R_{+}(q, \lambda) F(z, \lambda)=F(z, \lambda) r_{+}(q, z, \lambda) .
$$

Substituting (3.1.1) into this equation and rearranging terms yields

$$
e^{-\lambda^{-1} z A} h_{+}^{-1} R_{+} h_{+} e^{\lambda^{-1} z A}=e^{-\lambda \bar{z} A} p_{+}^{-1} r_{+} p_{+} e^{\lambda \bar{z} A} .
$$

Abbreviating $w_{+}(\lambda)=h_{+}^{-1} R_{+} h_{+}$and $V_{+}(z, \lambda)=e^{-\lambda \bar{z} A} p_{+}^{-1} r_{+} p_{+} e^{\lambda \bar{z} A}$, this is

$$
e^{-\lambda^{-1} z A} w_{+}(\lambda) e^{\lambda^{-1} z A}=V_{+}(z, \lambda) .
$$

The l.h.s. of (3.2.8) is

$$
Z=\left(\exp \left(-\lambda^{-1} z \operatorname{ad} A\right)\right) w_{+}(\lambda)
$$

Thus, (3.2.8) says that $Z$ has a Fourier expansion, where only non-negative powers of $\lambda$ occur. Expanding $w_{+}(\lambda)=w_{0}+w_{1} \lambda+w_{2} \lambda^{2}+\ldots$, altogether we have

$$
\begin{aligned}
\left(\exp \left(-\lambda^{-1} z \operatorname{ad} A\right)\right)\left(w_{0}+w_{1} \lambda+w_{2} \lambda^{2}+\ldots\right) & \\
& \text { does not contain any negative powers of } \lambda .
\end{aligned}
$$

Expanding (3.2.10) as a function of $z$, a straightforward induction on the power $N$ of $z$ shows

$$
\left[A, w_{+}(\lambda)\right]=0 .
$$


This implies

$$
V_{+}(z, \lambda)=V_{+}(0, \lambda)=w_{+}(\lambda)
$$

By the definition of $w_{+}$we have

$$
R_{+}=h_{+} w_{+} h_{+}^{-1} .
$$

Substituting (3.2.13) into (3.2.4) finally gives

$$
\chi(q, \lambda)=h_{+}(\lambda) e^{\left(\lambda^{-1} q-\lambda \bar{q}\right) A} w_{+}(\lambda) h_{+}^{-1}(\lambda) .
$$

3.3. This section is a "digression on $A$ ". We consider the matrix

$$
D=\frac{1}{\sqrt{2}}\left(\begin{array}{cc}
1 & 1 \\
-1 & 1
\end{array}\right)
$$

and verify

$$
D A D^{-1}=\sigma_{3} .
$$

Conjugating (3.2.7) by $D$ yields

$$
e^{-\lambda^{-1} z \sigma_{3}} \tilde{w}_{+} e^{\lambda^{-1} z \sigma_{3}} \in \Lambda_{r}^{+} \mathbf{S L}(2, \mathbb{C})_{\sigma},
$$

where $\tilde{w}_{+}=D w_{+} D^{-1}$. Thus, $\tilde{w}_{+}$and

$$
e^{-\lambda^{-1} z \sigma_{3}} \tilde{w}_{+} e^{\lambda^{-1} z \sigma_{3}}
$$

are defined at $\lambda=0$. If we set

$$
\tilde{w}_{+}=\left(\begin{array}{cc}
\tilde{w}_{a} & \tilde{w}_{b} \\
\tilde{w}_{c} & \tilde{w}_{d}
\end{array}\right),
$$

then the matrix in (3.3.4) has the form

$$
\left(\begin{array}{cc}
\tilde{w}_{a} & e^{-2 \lambda^{-1} z} \tilde{w}_{b} \\
e^{2 \lambda^{-1} z} \tilde{w}_{c} & \tilde{w}_{d}
\end{array}\right) .
$$

Therefore, if the off-diagonal entries of $\tilde{w}_{+}$do not vanish identically, then, for all $z \in \mathcal{D}$ where $\tilde{w}_{+}$is defined, the off-diagonal entries of the matrix in Eq. (3.3.4) have an essential singularity at $\lambda=0$. Therefore, $\tilde{w}_{b}=\tilde{w}_{c}=$ 0 , whence $\tilde{w}_{+}$commutes with $\sigma_{3}$ and $w_{+}=D^{-1} \tilde{w}_{+} D$ commutes with $A$, reproducing (3.2.11). 
The diagonalization (3.3.2) is also useful to represent $w_{+}$as an exponential. To this end we note, that $\tilde{w}_{+}=D w_{+} D^{-1}$ is a diagonal matrix $\tilde{w}_{+}=\operatorname{diag}\left(s_{+}, s_{+}^{-1}\right)$, with $s_{+}(\lambda) \in \mathbb{C}^{*}$ for $\lambda \in \overline{I^{(r)}}$. The map $\overline{I^{(r)}} \rightarrow \mathbb{C}^{*}$ can be factored through the universal cover $\mathbb{C}$ of $\mathbb{C}^{*}$ by standard topological reasons $[11$, Satz 4.9$]$, where the covering map $\mathbb{C} \rightarrow \mathbb{C} *$ is $\mu \mapsto e^{\mu}$. Thus, $s_{+}$can be written as $s_{+}=\exp \left(f_{+}\right)$, where

(3.3.7) $f_{+}: C_{r} \rightarrow \mathbb{C} \quad$ is odd and has a holomorphic extension to $I^{(r)}$.

Undoing the diagonalization of $w_{+}$we obtain

$$
w_{+}=e^{f_{+} A}
$$

Finally, in preparation of applications in the rest of this paper, we discuss the centralizer $C(A)$ of $A$ in $\Lambda_{r} \mathbf{S L}(2, \mathbb{C})_{\sigma}$ in some more detail. First we note

$$
C(A)=\left\{\alpha I+\beta A ; \alpha, \beta \in \mathcal{A}_{r}, \alpha^{2}-\beta^{2}=1\right\} .
$$

Here $\mathcal{A}_{r}$ denotes the algebra of functions on $C_{r}$ that defines the topology of the various loop groups considered in this paper.

The identity (3.3.9) is equivalent with the obvious fact, that $A$ is a regular semisimple matrix.

Conjugation with $D$ transforms $A$ into $\sigma_{3}$ and $C(A)$ into the centralizer of $\sigma_{3}$, i.e., into diagonal matrices. Thus,

$$
D(\alpha I+\beta A) D^{-1}=\operatorname{diag}(\alpha+\beta, \alpha-\beta) .
$$

This shows in particular that the eigenvalues of $\alpha I+\beta A \in C(A)$ are $\alpha+\beta$ and $\alpha-\beta$, and also $\alpha-\beta=(\alpha+\beta)^{-1}$, a trivial consequence of $\alpha^{2}-\beta^{2}=1$.

3.4. Next we consider the matrix

$$
H=e^{\left(\lambda^{-1} q-\lambda \bar{q}\right) A} w_{+}
$$

occuring in the description (3.2.14) of $\chi$. From (3.2.11) it follows, that $H$ commutes with $A$, i.e. (3.3.9) implies

$$
H=\alpha I+\beta A,
$$

This implies

$$
\alpha^{2}-\beta^{2}=1
$$

$$
H^{-1}=\alpha I-\beta A
$$


Since $q \neq 0$, we get from (3.4.1), that $H$ has an essential singularity at $\lambda=0$. From this it follows that

$$
\beta \not \equiv 0 \text {. }
$$

By the twisting condition we also know, that

$$
\alpha \text { is even in } \lambda \text { and } \beta \text { is odd in } \lambda \text {. }
$$

Since

$$
\chi=h_{+} H h_{+}^{-1}
$$

is holomorphic for $\lambda \in \mathbb{C}^{*}$, taking the trace of $\chi$ and $\chi^{2}$ yields

$$
\alpha \text { and } \beta^{2} \text { have holomorphic extensions to } \mathbb{C}^{*} \text {. }
$$

We note, that (3.4.1) and (3.4.2) imply

$$
\beta \text { has a holomorphic extension to } 0<|\lambda|<r \text {. }
$$

Remark. In general, $\beta$ will not have a holomorphic extension to $\mathbb{C}^{*}$. However, if $\beta$ does have a holomorphic extension to $\mathbb{C}^{*}$, then $H$ is also defined on $\mathbb{C}^{*}$.

Finally, we know from (3.3.8) that $w_{+}=\exp \left(f_{+} A\right)$. As a consequence, altogether we have

$$
H=e^{p A}, \quad p=\lambda^{-1} q-\lambda \bar{q}+f_{+} .
$$

In particular, with $f_{+}$also $p$ is odd in $\lambda$.

3.5. It will be convenient to consider the matrices

$$
\begin{gathered}
S=h_{+} A h_{+}^{-1}=\left(\begin{array}{ll}
a & b \\
c & d
\end{array}\right), \\
\hat{S}=\beta S=\left(\begin{array}{ll}
\hat{a} & \hat{b} \\
\hat{c} & \hat{d}
\end{array}\right) .
\end{gathered}
$$

In view of (3.4.2) and (3.4.7) we thus have

$$
\chi=\alpha I+\beta S=\alpha I+\hat{S}
$$

and (3.4.4) implies

$$
\chi^{-1}=\alpha I-\beta S=\alpha I-\hat{S} .
$$


Since, by Theorem $2.6, \chi$ is holomorphic on $\mathbb{C}^{*}$, we have

$$
\hat{a}, \hat{b}, \hat{c}, \hat{d} \text { are holomorphic on } \mathbb{C}^{*} \text {. }
$$

Clearly, we have $\operatorname{tr} S=0$ and $\operatorname{tr} \hat{S}=0$, whence

$$
d=-a, \quad \hat{d}=-\hat{a} .
$$

Also, in view of (3.4.6), the twisting condition for $\Lambda_{r} \mathbf{S L}(2, \mathbb{C})_{\sigma}$ implies

$$
\hat{a}, b, c \text { are even in } \lambda, a, \hat{b}, \hat{c} \text { are odd in } \lambda \text {. }
$$

Two important conditions still need to be evaluated: $S^{2}=I$ and $\left.\chi\right|_{S^{1}}$ unitary. The first condition is in view of (3.5.6) equivalent with

$$
a^{2}+b c=1 \text {. }
$$

Since $\hat{S}=\beta S$, we also have

$$
\hat{a}^{2}+\hat{b} \hat{c}=\beta^{2} .
$$

The second condition is in view of Section 2.8 and (3.4.4) equivalent with

$$
\begin{gathered}
\alpha \text { is defined on } \mathbb{C}^{*} \text { and } \alpha=\alpha^{*}, \\
\hat{S} \text { is defined on } \mathbb{C}^{*} \text { and } \hat{S}^{*}=-\hat{S} .
\end{gathered}
$$

In particular, (3.5.11) is equivalent with

$$
\hat{a}^{*}=-\hat{a}, \quad \hat{b}^{*}=-\hat{c} \quad \text { for } \lambda \in \mathbb{C}^{*} .
$$

Next we consider the squares of $\alpha, \beta, a, b, c$ and $\hat{a}, \hat{b}, \hat{c}$. First we note

$$
\alpha^{2} \text { and } \beta^{2} \text { are holomorphic on } \mathbb{C}^{*} \text { and real on } S^{1} \text {. }
$$

This follows for $\alpha^{2}$ from (3.5.10) and for $\beta^{2}$ from (3.4.3). Next, (3.5.12) implies

$$
\hat{a}^{2} \text { is holomorphic on } \mathbb{C}^{*} \text { and non-positive on } S^{1} \text {. }
$$

Substituting this and (3.5.12) into (3.5.9) gives

$$
\beta^{2} \text { is non-positive on } S^{1} \text {. }
$$


Since $\hat{a}=\beta a$, we know $\hat{a}^{2}=\beta^{2} a^{2}$. In particular, $a^{2}=\frac{\hat{a}^{2}}{\beta^{2}}$ is meromorphic on $\mathbb{C}^{*}$ and real on $S^{1}$. Since $a^{2}$ is by definition also holomorphic at $\lambda=0$, it follows that $a^{2}$ is also finite at $\lambda=\infty$. Thus

$$
a^{2} \text { is a rational function, real and non-negative on } S^{1}
$$

and finite at 0 and $\infty$.

For $b^{2}$ and $c^{2}$ one argues similarly. E.g. $b^{2}=\frac{\hat{b}^{2}}{\beta^{2}}$ is clearly meromorphic on $\mathbb{C}^{*}$ and is also, by the definition of $b$, holomorphic at $\lambda=0$. From (3.5.12) we obtain that $\left(b^{2}\right)^{*}=\frac{\left(\hat{b}^{2}\right)^{*}}{\beta^{2}}=\frac{\hat{c}^{2}}{\beta^{2}}=c^{2}$ is also holomorphic at $\lambda=0$. This shows, that $b^{2}$ is meromorphic on $\mathbb{C P}_{1}$ and thus rational. Altogether we have shown

$$
b^{2} \text { and } c^{2} \text { are rational and finite at } 0 \text { and } \infty .
$$

Moreover,

$$
\left(b^{2}\right)^{*}=c^{2} .
$$

Next, from (3.5.1) we see that $a(\lambda=0)=0$ and $b(\lambda=0)=c(\lambda=0)^{-1}$. Since $a$ is odd in $\lambda$, we obtain

$$
b(\lambda=0) c(\lambda=0)=1 .
$$

We also note that the relations

$$
\hat{a}^{2}=\beta^{2} a^{2}, \quad \hat{b}^{2}=\beta^{2} b^{2}, \quad \hat{c}^{2}=\beta^{2} c^{2}
$$

show that $a^{2}, b^{2}$, and $c^{2}$ can have poles only where $\beta^{2}$ has a zero.

Finally, from (3.5.11) we obtain $(\beta b)^{*}=-(\beta c)$. Hence (3.5.8) implies

$$
\beta^{2}=\beta^{2} a^{2}+\beta b \cdot \beta c=\beta^{2} a^{2}-(\beta b)(\beta b)^{*} .
$$

Therefore, on $S^{1}$ we obtain $\beta^{2}\left(a^{2}-1\right)=|\beta b|^{2}$. Since $\beta^{2}$ is non-positive on $S^{1}$ by (3.5.15), and $\beta^{2} \not \equiv 0$ by (3.4.5), we have $a^{2}-1 \leq 0$. Thus,

$$
0 \leq a^{2}(\lambda) \leq 1 \quad \text { for } \lambda \in S^{1} .
$$

3.6. In the last section we considered the matrix $S=h_{+} A h_{+}^{-1}=$ $\left(\begin{array}{ll}a & b \\ c & d\end{array}\right)$ and we listed properties of $a, b, c, d$. In the rest of this paper we 
will characterize $q \in \operatorname{Sym}(\Psi)$ in terms of $a, b, c, d$. Let us denote by $\mathcal{A}_{r}^{+}$, $0<r<1$, the subalgebra of those functions in $\mathcal{A}_{r}$ which can be extended holomorphically to $I^{(r)}$. Then $a, b, c, d \in \mathcal{A}_{r}^{+}$. To make sure, that there is also an $h_{+}$satisfying (3.5.1), producing $F$ - and thus $\Psi$ - for which $q \in \operatorname{Sym}(\Psi)$, we prove:

Theorem. Let $a, b, c, d \in \mathcal{A}_{R}^{+}, 0<R<1$, where $a, d$ are odd and $b, c$ are even. Then $S=\left(\begin{array}{ll}a & b \\ c & d\end{array}\right)$ is of the form $S=h_{+} A h_{+}^{-1}$ for some $0<r \leq R$ and $h_{+} \in \Lambda_{r}^{+} \boldsymbol{S L}(2, \mathbb{C})_{\sigma}$ iff

$$
\begin{gathered}
d=-a, \\
a^{2}+b c=1, \\
b(\lambda=0) \neq 0 .
\end{gathered}
$$

Proof. " $\Rightarrow$ " This direction was already proved in the last section.

" $\Leftarrow$ " Using the matrix $D$ introduced in (3.3.1), we obtain

$$
S=h_{+} A h_{+}=h_{+} D^{-1} \sigma_{3} D h_{+}^{-1}=Y \sigma_{3} Y^{-1}
$$

where

$$
Y=h_{+} D^{-1}
$$

A straightforward formal calculation shows, that

$$
Y=\frac{i}{\sqrt{2}}\left(\begin{array}{cc}
\sqrt{b} & \sqrt{b} \\
\frac{1-a}{\sqrt{b}} & -\frac{1+a}{\sqrt{b}}
\end{array}\right) \cdot\left(\begin{array}{cc}
x(\lambda) & 0 \\
0 & x(\lambda)^{-1}
\end{array}\right) .
$$

Clearly, $Y$ is (formally) a matrix of eigenvectors of $S$. We get for $h_{+}=Y D$ again formally

$(3.6 .7) h_{+}=\frac{i}{2 \sqrt{b}}\left(\begin{array}{cc}\left(x-x^{-1}\right) b & \left(x+x^{-1}\right) b \\ \left(x+x^{-1}\right)-\left(x-x^{-1}\right) a & \left(x-x^{-1}\right)-\left(x+x^{-1}\right) a\end{array}\right)$.

For this matrix to be defined in our setup we need that the diagonal entries are even in $\lambda$ and the off-diagonal entries are odd in $\lambda$ and that everything is defined for all $0 \leq|\lambda| \leq r$, where $0<r \leq R$ is chosen appropriately.

Since $b$ is even and $b(\lambda=0) \neq 0, \sqrt{b}$ is defined in a sufficiently small neighbourhood of $\lambda=0$ and is even there. So what we need to achieve is 
that $\left(x-x^{-1}\right) \sqrt{b}$ is even, $\left(x+x^{-1}\right) \sqrt{b}$ is odd, $\frac{\left(x+x^{-1}\right)-\left(x-x^{-1}\right) a}{\sqrt{b}}$ is odd, and $\frac{\left(x-x^{-1}\right)-\left(x+x^{-1}\right) a}{\sqrt{b}}$ is even, and all these functions are defined on a sufficiently small neighbourhood of $\lambda=0$. This is equivalent with: $x-x^{-1}$ is even and $x+x^{-1}$ is odd and $x$ is invertible in a neighbourhood of $\lambda=0$. It is straightforward to verify, that $x=-i \sqrt{\frac{1+a}{1-a}}$ satisfies these conditions. In this case

$$
h_{+}=\frac{1}{\sqrt{c}}\left(\begin{array}{ll}
1 & a \\
0 & c
\end{array}\right)
$$

and $r$ is determined by $\sqrt{c}$.

3.7. Let us collect the necessary conditions we have derived in Sections $3.2-3.5$ :

Theorem. Let $\Psi: \mathbb{C} \rightarrow \mathbb{R}^{3}$ be a CMC-immersion with extended frame $F(z, \lambda)$, s.t. $F(z, \lambda)$ is given by dressing the cylinder under the $r$-dressing (2.5.1) with some $h_{+} \in \Lambda_{r}^{+} \boldsymbol{S L}(2, \mathbb{C})_{\sigma}$. Assume also, that for $q \in \mathbb{C}, q \neq 0$, $F(z+q, \lambda)=\chi(\lambda) F(z, \lambda)$, i.e., $q \in \operatorname{Sym}(\Psi)$. Define $h_{+} A h_{+}^{-1}=\left(\begin{array}{ll}a & b \\ c & d\end{array}\right)$.

Then $d=-a$ and the functions $a(\lambda), b(\lambda)$, and $c(\lambda)$ are in $\mathcal{A}_{r}^{+}$and satisfy the following conditions:

a) $a^{2}, b^{2}, c^{2}$ are rational,

b) $a$ is odd in $\lambda, b$ and $c$ are even in $\lambda$,

c) $a^{2}+b c=1$.

d) $a^{2}$ is real on $S^{1}$ and $0 \leq a^{2} \leq 1$ on $S^{1}$,

e) $c^{2}=\left(b^{2}\right)^{*}$.

Furthermore, there exists an odd function $f_{+}$in $\mathcal{A}_{r}^{+}$, s.t. with $\alpha=\cosh (p)$, $\beta=\sinh (p), p=\lambda^{-1} q-\lambda \bar{q}+f_{+}$, we have:

$\left.\mathbf{a}^{\prime}\right) \quad \alpha$ and $\beta^{2}$ are defined on $\mathbb{C}^{*}$,

$\left.\mathbf{b}^{\prime}\right) \quad \alpha$ and $\beta^{2}$ are real on $S^{1}$,

$\left.\mathbf{c}^{\prime}\right) \quad \beta^{2}$ is non-positive on $S^{1}$,

$\left.\mathbf{d}^{\prime}\right)$ the functions $\beta a, \beta b$, and $\beta c$ extend holomorphically to $\mathbb{C}^{*}$.

The matrix function $\chi(\lambda)$ is given by $\chi=\alpha I+\beta h_{+} A h_{+}^{-1}$.

Proof. By the results of the last sections we know for the functions $a, b, c, d$, defined by $S=h_{+} A h_{+}^{-1}=\left(\begin{array}{ll}a & b \\ c & d\end{array}\right)$ :

- $d=-a:(3.5 .6)$,

- $a^{2}, b^{2}, c^{2}$ are rational functions: (3.5.16), (3.5.17), 
- $\quad a$ is odd, $b$ and $c$ are even in $\lambda$ : (3.5.7),

- $a^{2}+b c=1:(3.5 .8)$,

- $a^{2}$ is real on $S^{1}$ and $0 \leq a^{2} \leq 1$ on $S^{1}$ : (3.5.16), (3.5.23),

- $c^{2}=\left(b^{2}\right)^{*}:(3.5 .18)$.

Since $q \in \operatorname{Sym}(\Psi)$ we have $F(z+q, \lambda)=\chi(\lambda) F(z, \lambda)$ by Theorem 2.6. Moreover, $\chi(\lambda)=h_{+} e^{p A} h_{+}^{-1}$, where $p=\lambda^{-1} q-\lambda \bar{q}+f_{+}$by (3.4.7) and (3.4.10). Thus $\chi=\alpha I+\beta S$, where $\alpha=\cosh (p), \beta=\sinh (p)$ and

- $\quad \alpha$ and $\beta^{2}$ are defined on $\mathbb{C}^{*}$ : (3.4.8),

- $\alpha$ and $\beta^{2}$ are real on $S^{1}$ : (3.5.10), (3.5.13),

- $\beta^{2}$ is non-positive on $S^{1}$ : (3.5.15).

Finally, $\hat{a}=\beta a, \hat{b}=\beta b$, and $\hat{c}=\beta c$ extend holomorphically to $\mathbb{C} *$ by (3.5.5).

3.8. We have seen in Section 3.6 under what conditions on $a, b, c$, and $d$ we can find an $h_{+} \in \Lambda_{r}^{+} \mathbf{S L}(2, \mathbb{C})_{\sigma}$, s.t. $\left(\begin{array}{ll}a & b \\ c & d\end{array}\right)=h_{+} A h_{+}^{-1}$. This then defines a CMC-immersion $\Psi$ via dressing of the trivial solution with $h_{+}$. In this section we characterize those $a, b, c, d, \alpha, \beta$ such that a given $q \in \mathbb{C}^{*}$ is in $\operatorname{Sym}(\Psi)$.

Theorem. Let there be given three even rational functions $a^{2}(\lambda), b^{2}(\lambda)$, and $c^{2}(\lambda)$, which satisfy the following conditions:

a) $a^{2}$ is real on $S^{1}$ and $0 \leq a^{2} \leq 1$ on $S^{1}$,

b) $c^{2}=\left(b^{2}\right)^{*}$.

c) There exists an $0<r<1$, s.t. the restrictions of $a^{2}, b^{2}$, and $c^{2}$ to $C_{r}$ are the squares of functions $a, b, c$ in $\mathcal{A}_{r}^{+}$,

d) $a$ is odd, $b$ and $c$ are even in $\lambda$,

e) $a^{2}+b c=1$.

In addition, with $r$ as in $\mathrm{c})$, we assume that there exists an odd function $f_{+}$in $\mathcal{A}_{r^{\prime}}^{+}, 0<r^{\prime} \leq r$, such that for $p=\lambda^{-1} q-\lambda \bar{q}+f_{+}, \alpha=\cosh (p)$, $\beta=\sinh (p), q \in \mathbb{C}^{*}$, we have:

$\left.\mathbf{a}^{\prime}\right) \quad \alpha$ and $\beta^{2}$ are defined and holomorphic on $\mathbb{C}^{*}$,

$\left.\mathbf{b}^{\prime}\right) \quad \alpha$ and $\beta^{2}$ are real on $S^{1}$,

$\left.\mathbf{c}^{\prime}\right) \quad \beta^{2}$ is non-positive on $S^{1}$.

$\left.\mathbf{d}^{\prime}\right)$ The functions $\beta a, \beta b$, and $\beta c$ extend holomorphically to $\mathbb{C}$.

Then there exists $0<r^{\prime \prime} \leq r^{\prime}$ and $h_{+} \in \Lambda_{r^{\prime \prime}}^{+} \boldsymbol{S L}(2, \mathbb{C})_{\sigma}$, such that $h_{+} A h_{+}^{-1}=$ $\left(\begin{array}{cc}a & b \\ c & -a\end{array}\right)$. Moreover, for the extended frame $F(z, \lambda)$ defined by $h_{+} e^{\left(\lambda^{-1} z-\lambda \bar{z}\right) A}$ $=F(z, \lambda) p_{+}(z, \lambda),|\lambda|=r^{\prime \prime}$, we have $F(z+q, \lambda)=\chi(\lambda) F(z, \lambda)$, where $\chi=$ 
$\alpha I+\beta h_{+} A h_{+}^{-1}$ is holomorphic on $\mathbb{C}^{*}$ and takes values in $\boldsymbol{S U}(2)$ on $S^{1}$. In particular, $q \in \operatorname{Sym}(\Psi)$ for the $\mathrm{CMC}$-immersion $\Psi$ associated with $F(z, \lambda)$ via Sym's formula.

Proof. Assume, that we have functions $a^{2}, b^{2}, c^{2}$ and $f_{+}, p, \alpha, \beta$, such that a)-e), a')-d') are satisfied. We first want to apply Theorem 3.6. We set $d=-a$ and know (3.6.2) by e). Since $a, b, c$ are defined at $\lambda=0$ and since $a$ is odd we have $a(0)=0$, whence $b(0) \neq 0$. Thus, by Theorem 3.6, there exists some $0<r^{\prime \prime} \leq r^{\prime}<1$ and some $h_{+} \in \Lambda_{r^{\prime \prime}}^{+} \mathbf{S L}(2, \mathbb{C})_{\sigma}$, s.t. $S=$ $h_{+} A h_{+}^{-1}=\left(\begin{array}{cc}a & b \\ c & -a\end{array}\right)$. Next we consider the extended frame defined by the $r$-dressing $h_{+} e^{\left(\lambda^{-1} z-\lambda \bar{z}\right) A}=F(z, \lambda) p_{+}(z, \lambda)$ of the cylinder. Recall that we use in this paper the unique Iwasawa splitting discussed in Section 2.2. We also set $\chi=h_{+} e^{p A} h_{+}^{-1}=\alpha I+\beta S$. From a') and d') it follows, that $\chi$ is defined and holomorphic on $\mathbb{C}^{*}$. Since with $f_{+}$also $p$ is an odd function in $\lambda, p A \in \Lambda_{r^{\prime \prime}} \mathbf{s l}(2, \mathbb{C})_{\sigma}$, whence $\chi \in \Lambda_{r^{\prime \prime}} \mathbf{S L}(2, \mathbb{C})_{\sigma}$. As outlined in Section 3.5, $\chi$ is unitary on $S^{1}$, iff (3.5.10) and (3.5.11) are satisfied. But (3.5.10) follows from a'), b'), and the first part of (3.5.11) is just d'). The second condition is $(\beta a)^{*}=-(\beta a)$ and $(\beta c)^{*}=-(\beta b)$. To verify this condition we square $\beta a$, $\beta b$, and $\beta c$ and obtain $\left((\beta a)^{2}\right)^{*}=(\beta a)^{2}$ and $\left((\beta c)^{2}\right)^{*}=(\beta b)^{2}$, since $\beta^{2}$ and $a^{2}$ are real by b'), and $\left(c^{2}\right)^{*}=b^{2}$ by $\left.\mathrm{b}\right)$. Hence $(\beta a)^{*}= \pm \beta a$ and $(\beta c)^{*}= \pm \beta b$. If $(\beta a)^{*}=\beta a$, then $\beta a$ is real on $S^{1}$ and $\beta^{2} a^{2}=(\beta a)^{2}$ is non-negative on $S^{1}$. But a) and c') imply that $\beta^{2} a^{2}$ is non-positive on $S^{1}$. The only possibility for both conditions to hold is $\beta a=0$ on $S^{1}$. But in this case, of course, also $(\beta a)^{*}=-\beta a$ as desired. For the remaining case we consider e) and obtain $\beta^{2}=\beta^{2} a^{2}+(\beta b)(\beta c)$. If $(\beta c)^{*}=+\beta b$, then $\beta^{2}=\beta^{2} a^{2}+|\beta c|^{2}$ on $S^{1}$. Hence $|\beta c|^{2}=\beta^{2}\left(1-a^{2}\right)$ implies $\beta^{2} \equiv 0$ or $1-a^{2} \leq 0$. The first case is not possible in view of the form of $p$. The second case yields in view of a), that $a^{2} \equiv 1$ on $S^{1}$. Hence $a= \pm 1$ on $\mathbb{C}$, a contradiction, since $a(0)=0$. Thus $(\beta c)^{*}=-\beta b$ as required.

Finally, we show $q \in \operatorname{Sym}(\Psi)$. To this end we multiply $\chi=h_{+}(\alpha I+$ $\beta A) h_{+}^{-1}$ from the right with $h_{+} e^{\left(\lambda^{-1} z-\lambda \bar{z}\right) A}$ and obtain

$$
\chi h_{+} e^{\left(\lambda^{-1} z-\lambda \bar{z}\right) A}=h_{+} e^{\left(\lambda^{-1}(z+q)-\lambda(\overline{z+q})\right) A} e^{f_{+}} .
$$

Using the definition of $F_{+}(z, \lambda)$ thus gives

$$
\chi(\lambda) F(z, \lambda) p_{+}(z, \lambda)=F(z+q, \lambda) p_{+}(z+q, \lambda) e^{f_{+}} .
$$

This shows,

$$
F(z+q, \lambda)=\chi(\lambda) F(z, \lambda)
$$


since the Iwasawa splitting chosen is unique and $\chi$ is unitary.

3.9. It remains to state the closing conditions Eq. (2.7.5) in terms of the functions used in Theorem 3.8. These turn out to be of a very simple form.

Theorem. Let $\Psi: \mathbb{C} \rightarrow \mathbb{R}^{3}$ be a CMC-immersion in the $r$-dressing orbit of the cylinder with associated family $\left\{\Psi_{\lambda}, \lambda \in S^{1}\right\}$. Let $q \in \operatorname{Sym}(\Psi)=$ $\operatorname{Sym}\left(\Psi_{\lambda}\right), q \neq 0$, and $\chi(\lambda)$ and $\beta^{2}(\lambda)$ as in Theorem 3.7. Restrict $\lambda$ to $S^{1}$ and denote by $(\cdot)^{\prime}$ differentiation w.r.t. $\theta$, where $\lambda=e^{i \theta}$. Then for $\lambda_{0} \in S^{1}$ we have:

a) $\chi\left(\lambda_{0}\right)= \pm I$ iff $\beta^{2}$ vanishes at $\lambda_{0}$.

b) $\chi\left(\lambda_{0}\right)= \pm I$ and $\chi^{\prime}\left(\lambda_{0}\right)=0$ iff $\beta^{2}$ vanishes at least to fourth order at $\lambda_{0}$.

Proof. Let us first prove a): Let us define $a, b, c$ and $f_{+}, p, \alpha, \beta$ as in Theorem 3.7. Then $\chi=\alpha I+\beta\left(\begin{array}{cc}a & b \\ c & -a\end{array}\right)$. By c) in Theorem 3.7, we have $\operatorname{det} \chi=$ $\alpha^{2}-\beta^{2}=1$. Therefore, $\chi\left(\lambda_{0}\right)= \pm I$ implies $\alpha\left(\lambda_{0}\right)=\frac{1}{2} \operatorname{tr}\left(\chi\left(\lambda_{0}\right)\right)= \pm 1$ and $\beta^{2}\left(\lambda_{0}\right)=0$.

Conversely, assume $\beta^{2}\left(\lambda_{0}\right)=0$ for $\lambda_{0} \in S^{1}$. Then $\alpha\left(\lambda_{0}\right)= \pm 1$. By d) in Theorem 3.7 we have that $a^{2}$ has no poles on $S^{1}$. By c) and e) in Theorem 3.7, also $b^{2}$ and $c^{2}$ are defined everywhere on $S^{1}$. This shows, that

$$
\beta^{2}\left(\lambda_{0}\right) a^{2}\left(\lambda_{0}\right)=\beta^{2}\left(\lambda_{0}\right) b^{2}\left(\lambda_{0}\right)=\beta^{2}\left(\lambda_{0}\right) c^{2}\left(\lambda_{0}\right)=0 .
$$

This together with $\alpha\left(\lambda_{0}\right)= \pm 1$ implies $\chi\left(\lambda_{0}\right)= \pm I$.

Now we prove b). Let us assume, that $\chi\left(\lambda_{0}\right)= \pm I$ and $\chi^{\prime}\left(\lambda_{0}\right)=0$. By Theorem 3.7 we have

$$
\chi^{\prime}=\left(\begin{array}{cc}
\alpha^{\prime}+(\beta a)^{\prime} & (\beta b)^{\prime} \\
(\beta c)^{\prime} & \alpha^{\prime}-(\beta a)^{\prime}
\end{array}\right) .
$$

Therefore, and by the proof of part a), $(\beta a)^{2}=\beta^{2} a^{2},(\beta b)^{2}=\beta^{2} b^{2}$, and $(\beta c)^{2}=\beta^{2} c^{2}$ vanish at least to fourth order at $\lambda_{0}$. By c), d), and e) in Theorem 3.7 we know, that on $S^{1} b^{2}, c^{2}$, and $a^{2}=1-\left|b^{2}\right|^{2}$ are defined and cannot vanish simultaneously. Therefore, $\beta^{2}$ vanishes at least to fourth order at $\lambda_{0}$.

Conversely, assume $\beta^{2}$ vanishes at least to fourth order at $\lambda_{0}$. Then we have already shown, that $\chi\left(\lambda_{0}\right)= \pm I, \alpha\left(\lambda_{0}\right)= \pm 1$. By differentiating $\alpha^{2}-\beta^{2}=1$, we get $\alpha^{\prime}\left(\lambda_{0}\right)= \pm \beta\left(\lambda_{0}\right) \beta^{\prime}\left(\lambda_{0}\right)=0$. Also, $a^{2}, b^{2}$, and $c^{2}$ are holomorphic on $S^{1}$. Therefore, $\beta^{2} a^{2}, \beta^{2} b^{2}, \beta^{2} c^{2}$ all vanish at least to fourth order at $\lambda_{0}$. This shows, that $\beta a, \beta b, \beta c$ vanish at least to second order at $\lambda_{0}$. From this and (3.9.2), the claim follows. 
Corollary. Let $\Psi$ and $\Psi_{\lambda}$ be defined as in the theorem above and let $q \in$ $\operatorname{Sym}(\Psi), q \neq 0$. Then the set $\Omega_{q}=\left\{\lambda \in S^{1} \mid q \in \operatorname{Per}\left(\Psi_{\lambda}\right)\right\}$ is discrete.

Proof. We define $p=\lambda^{-1} q-\lambda \bar{q}+f_{+}, \alpha=\cosh (p)$ and $\beta=\sinh (p)$ as in Theorem 3.7. By Theorem 3.9 we know, that $\beta^{2}$ vanishes on the set $\Omega_{q}$. Therefore, if $\Omega_{q}$ has an accumulation point, then the holomorphic function $\beta^{2}$ vanishes everywhere on $\mathbb{C}^{*}$, contradicting (3.4.5).

Remark. The corollary does not exclude the possibility that there are surfaces in the generalized dressing orbit of the standard cylinder, whose associated family consists entirely of periodic surfaces. The members of the associated family of the standard cylinder itself, for example, are cylinders for all $\lambda \in S^{1}$. But the corollary implies, that the translations under which the surfaces are periodic depend on the spectral parameter. In the case of the cylinder this can easily be checked by a direct calculation.

\section{Hyperelliptic curves.}

Let $\Psi: \mathbb{C} \rightarrow \mathbb{R}^{3}$ be a CMC-immersion in the $r$-dressing orbit of the cylinder with associated family $\left\{\Psi_{\lambda} ; \lambda \in S^{1}\right\}$. I.e., if $F(z, \lambda)$ is the extended frame of $\Psi$, then there exists $0<r<1$ and $h_{+} \in \Lambda_{r}^{+} \mathbf{S L}(2, \mathbb{C})_{\sigma}$, such that $F(z, \lambda)$ is given by (3.1.1). In this chapter we also assume, that $\Psi$ has a periodic metric, i.e., that there exists $q \in \operatorname{Sym}(\Psi)=\operatorname{Sym}\left(\Psi_{\lambda}\right), q \neq 0$. As we have seen in Theorem 3.7 and Theorem 3.8, we can formulate the periodicity conditions for the metric of a CMC-immersion in terms of scalar functions $a, b, c$ given by $h_{+} A h_{+}^{-1}=\left(\begin{array}{cc}a & b \\ c & -a\end{array}\right)$. These functions are holomorphic in a neighbourhood $I^{(r)}$ of $\lambda=0$. In this section we will introduce a nonsingular hyperelliptic curve, on which $a, b$ and $c$ can be viewed as meromorphic functions. From now on, the symbols $a, b, c, f_{+}, p, \alpha, \beta$ refer to the functions introduced in Theorem 3.7.

4.1. First we define the new variable $\nu=\lambda^{2}$. The functions $a^{2}(\lambda)$, $b^{2}(\lambda)$, and $c^{2}(\lambda)$ are by Theorem 3.7 rational and even. We will regard $a^{2}$, $b^{2}$, and $c^{2}$ as rational functions of $\nu$. Since by b) in Theorem 3.7, $a$ is an odd function in $\lambda$, either $a=0$ (which corresponds to the standard cylinder) or $a^{2}(\lambda)$ has a zero of order $2(2 n-1), n>0$, at $\lambda=0$. As a function in $\nu$, $a^{2}$ has therefore a zero of odd order $2 n-1$ at $\nu=0$. Let $\nu_{1}, \ldots, \nu_{k}$ be the points in the $\nu$-plane where $a^{2}$ has a pole of odd order, and let $\nu_{k+1}, \ldots, \nu_{k+l}$ be the points in the $\nu$-plane away from $\nu=0$ where $a^{2}$ has a zero of odd order.

Lemma. None of the points $\nu_{1}, \ldots, \nu_{k+l} \in \mathbb{C} *$ defined above lies on the unit circle. 
Proof. We have to show, that $a^{2}(\nu)$ has neither a pole nor a zero of odd order on $S^{1}$. In fact, it doesn't matter here, if we view $a^{2}$ as a function of $\lambda$ or $\nu$. By d) in Theorem 3.7, we know, that $a^{2}$ has no poles on $S^{1}$. By c) and e) in Theorem 3.7, we have $\left(1-a^{2}\right)^{2}=\left|b^{2}\right|^{2}$ on $S^{1}$, which shows that $b^{2}$ and $c^{2}=\left(b^{2}\right)^{*}$ are defined on $S^{1}$ and also, that $a^{2}, b^{2}$, and $c^{2}$ cannot vanish simultaneously on $S^{1}$. If $a^{2}$ has a zero of odd order at $\lambda_{0} \in S^{1}$, then $b^{2}\left(\lambda_{0}\right) \neq 0$. By d') of Theorem 3.7, $(\beta a)^{2}$ and $(\beta b)^{2}$ are squares of holomorphic functions on $\mathbb{C}^{*}$. Thus, the function $\beta^{2}=\frac{(\beta a)^{2}}{a^{2}}=\frac{(\beta b)^{2}}{b^{2}}$ has both a zero of odd order and of even order at $\lambda_{0}$. This implies $\beta \equiv 0$, contradicting (3.4.5).

Proposition. Let $k, l$ and $\nu_{1}, \ldots, \nu_{k+l}$ be defined as above. Then $g=$ $\frac{1}{2}(k+l)$ is an integer and we can order the points $\nu_{1}, \ldots, \nu_{2 g}$, such that

$$
\nu_{2 n}=\tau\left(\nu_{2 n-1}\right), \quad\left|\nu_{2 n-1}\right|<1, \quad n=1, \ldots, g .
$$

Proof. By Lemma 4.1, we have $\left|\nu_{2 n-1}\right| \neq 1$ for $n=1, \ldots, k+l$. Since $a^{2}(\nu)$ is real on $S^{1}$, we have using Section 2.8, that the set $B=\left\{\nu_{1}, \ldots, \nu_{k+l}\right\}$ is invariant under the antiholomorphic involution $\tau: \nu \rightarrow \bar{\nu}^{-1}$. Since $\tau$ has no fixed points off the unit circle, we get that $B$ consists of pairs $\left(\nu_{n}, \tau\left(\nu_{n}\right)\right)$. This shows, that $k+l$ is even, whence $g=\frac{1}{2}(k+l)$ is an integer, and that we can order $\left\{\nu_{1}, \ldots, \nu_{2 g}\right\}$, such that (4.1.1) holds.

In the following we will order the points $\nu_{1}, \ldots, \nu_{2 g}$ always such that (4.1.1) holds.

Consider the algebraic equation

$$
\mu^{2}=\nu \prod_{k=1}^{2 g}\left(\nu-\nu_{k}\right) .
$$

Theorem. The plane affine curve $\tilde{\mathcal{C}}$ defined by (4.1.2) can be uniquely extended to a compact Riemann surface $\mathcal{C}$ of genus $g$. The meromorphic function $\nu: \tilde{\mathcal{C}} \rightarrow \mathbb{C}$ extends to a holomorphic map $\pi: \mathcal{C} \rightarrow \mathbb{C} \mathrm{P}_{1}$ of degree 2 . The branchpoints of $\pi$ are the roots of $\mu^{2}$ and the point $\infty$.

Proof. The proof follows immediately from [19, Lemma III.1.7] since $\mu^{2}$ has odd degree.

In other words, (4.1.2) is a (nonsingular) hyperelliptic curve, obtained by compactifying the plane affine curve $\tilde{\mathcal{C}}=\mathcal{C} \backslash\left\{P_{\infty}\right\}$, where $P_{\infty}=\pi^{-1}(\infty) \in \mathcal{C}$ is a single point. 
Remark. 1. The rational functions $a^{2}, b^{2}$ and $c^{2}$ in Theorem 3.7 are defined in terms of the extended frame $F(z, \lambda)$ of $\Psi$. I.e., they are defined for the whole associated family, not only for $\Psi$. Therefore, also the hyperelliptic curve $\mathcal{C}$ is associated to the whole family $\left\{\Psi_{\lambda} ; \lambda \in S^{1}\right\}$. This corresponds to the fact that $\operatorname{Sym}(\Psi)=\operatorname{Sym}\left(\Psi_{\lambda}\right)$.

2. In Proposition 4.4 it will be shown, that $a(\nu)=\sqrt{a^{2}(\nu)}$ can be lifted to a nonconstant meromorphic function on $\mathcal{C}$. It determines the complex structure of $\mathcal{C}$ uniquely (see [10, I.1.6], [11, Satz 8.9]).

4.2. $\quad$ On $\tilde{\mathcal{C}}=\mathcal{C} \backslash\left\{P_{\infty}\right\}, \nu$ and $\mu$ are holomorphic functions. Every point $P \in \mathcal{C} \backslash\left\{P_{\infty}\right\}$ is determined uniquely by the values of $\nu$ and $\mu$ at $P$ and can thus be identified with the pair $(\nu(P), \mu(P))$. Formally, we will also write $P_{\infty}=(\infty, \infty)$. In this notation we get $\pi(\nu, \mu)=\nu$. Let us define the hyperelliptic involution $I$ on $\mathcal{C}$ by

$$
I(\nu, \mu)=(\nu,-\mu)
$$

A point on $\mathcal{C}$ is a branchpoint iff it is mapped by $\pi$ to a branchpoint on $\mathbb{C} \mathrm{P}_{1}$. Clearly, the branchpoints of $\mathcal{C}$ are precisely the fixed points of $I$, i.e., the points $\left(\nu_{k}, 0\right), k=1, \ldots, 2 g, P_{0}=(0,0)$ and $P_{\infty}$.

Using the representation of $\mathcal{C}$ given in [19, Chapter III] it is easy to see that the functions $\mu$ and $\mu \nu^{-(g+1)}$ are local coordinates on $\mathcal{C}$ at $P_{0}$ and $P_{\infty}$, respectively. It will also be convenient to use the coordinate $\lambda=\lambda(\nu, \mu)$ on $\mathcal{C}$ at $P_{0}$ given by $\lambda(\nu, \mu)=\mu /\left(\prod_{j=1}^{2 g}\left(\nu-\nu_{j}\right)\right)^{\frac{1}{2}}$. In view of the definition of $\mathcal{C}$ we clearly have the relation $\lambda^{2}=\nu$ near $P_{0}$. In particular, since $\nu \circ I=\nu$ we obtain $\lambda \circ I= \pm \lambda$. The fact that $\lambda$ is injective finally implies $\lambda \circ I=-\lambda$.

Using the change of coordinates $(\nu, \mu) \rightarrow\left(\nu^{-1}, \mu \nu^{-(g+1)}\right)$ and the defining relation for $\mathcal{C}$ we can define similarly a coordinate $\lambda^{-1}$ near $P_{\infty}$ such that $\left(\lambda^{-1}\right)^{2}=\nu^{-1}$ and $\lambda^{-1} \circ I=-\lambda^{-1}$.

Let us investigate the set of meromorphic functions on $\mathcal{C}$. By [19], Proposition 1.10 , every meromorphic function on $\mathcal{C}$ can be uniquely written as

$$
f(\nu, \mu)=f_{1}(\nu)+f_{2}(\nu) \mu
$$

with two rational functions $f_{1}, f_{2}$.

Remark. It is clear from the representation (4.2.2) of meromorphic functions on $\mathcal{C}$, that each rational function $f_{1}(\nu)$ can be lifted to a meromorphic function on $\mathcal{C}$ by setting $f(\nu, \mu)=f_{1}(\nu)$. Clearly, then $f \circ I=f$ for such a function.

Conversely, if $f: \mathcal{C} \rightarrow \mathbb{C}$ is meromorphic, then it can be identified with a rational function $f_{1}(\nu)$ iff $f_{2}(\nu) \equiv 0$ in (4.2.2), i.e., iff it satisfies $f \circ I=f$. 
We will frequently use this identification of rational functions in $\nu$ with $I$ invariant meromorphic functions on $\mathcal{C}$.

4.3. Let us define

$$
\mathcal{S}=\pi^{-1}\left(S^{1}\right)=\left\{(\nu, \mu) \in \mathcal{C} ; \nu \in S^{1}\right\} .
$$

The set $\mathcal{S}$ is connected if $g$ is even, and has two connected components if $g$ is odd. Since $\mathcal{S}$ is contained in $\tilde{\mathcal{C}}$, we can identify it with a subset of $\mathbb{C}^{2}$. Using the antiholomorphic involution $\tau: \nu \rightarrow \bar{\nu}^{-1}$ defined in Section 2.8, we define the map $\tilde{\sigma}: \tilde{\mathcal{C}} \backslash\left\{P_{0}\right\} \rightarrow \tilde{\mathcal{C}} \backslash\left\{P_{0}\right\}$ by

$$
\tilde{\sigma}:(\nu, \mu) \longmapsto\left(\frac{1}{\bar{\nu}},\left(\frac{1}{\bar{\nu}}\right)^{(g+1)}\left(\prod_{j=1}^{2 g} \nu_{j}\right)^{\frac{1}{2}} \bar{\mu}\right) .
$$

We will choose the sign of the square root such that the points on $\mathcal{S}$ are fixed by $\tilde{\sigma}$.

The following is well known (see e.g. [9, 15]):

Theorem. The map $\tilde{\sigma}$ defined by (4.3.2) can be extended to an antiholomorphic involution $\hat{\sigma}$ on $\mathcal{C}$, which preserves the points of $\mathcal{S} \subset \mathcal{C}$.

Furthermore, $\hat{\sigma}$ commutes with the hyperelliptic involution and leaves invariant the set of branchpoints of $\mathcal{C}$.

Proof. Using Theorem 4.1 it is easily checked, that $\tilde{\sigma}$ defines an antiholomorphic involution on $\tilde{\mathcal{C}} \backslash\left\{P_{0}\right\}$ By using the coordinates $\mu$ and $\mu \nu^{-(g+1)}$ near $P_{0}$ and $P_{\infty}$ respectively, we get $\tilde{\sigma}(\mu)=c_{0} \bar{\mu}, c_{0}=\left(\prod_{j=1}^{2 g} \nu_{j}\right)^{\frac{1}{2}}$, in local coordinates, whence $\tilde{\sigma}$ extends to an antiholomorphic involution $\hat{\sigma}$ on $\mathcal{C}$, which maps $P_{0}$ to $P_{\infty}$. By the choice of the square root in (4.3.2), $\hat{\sigma}$ fixes the points on $\mathcal{S}$. $\hat{\sigma}$ clearly commutes with $I$. If $P$ is a branchpoint of $\mathcal{C}$, then $I(P)=P$. Therefore, $I(\hat{\sigma}(P))=\hat{\sigma}(I(P))=\hat{\sigma}(P)$ and $\hat{\sigma}(P)$ is also a branchpoint. Thus, $\hat{\sigma}$ leaves invariant the set of branchpoints of $\mathcal{C}$.

For a scalar function on $\mathcal{C}$ we also define

$$
f^{*}=\overline{f \circ \hat{\sigma}} .
$$

Since, by Proposition 4.3, $\hat{\sigma}$ fixes the points of $\mathcal{S}$, we get

Lemma. Let $f$ be a meromorphic function defined on a $\hat{\sigma}$-invariant subset of $\mathcal{C}$ which contains $\mathcal{S}$. Then $f$ is real on $\mathcal{S}$ iff $f^{*}=f$ and $f$ is purely imaginary on $\mathcal{S}$ iff $f^{*}=-f$.

4.4. Let us now investigate the properties of $a^{2}$ w.r.t. $\mathcal{C}$. 
Proposition. The rational function $a^{2}$ defined in Theorem 3.7 is of the form

$$
a^{2}(\nu)=f(\nu)^{2} \mu^{2}(\nu)
$$

where $f$ is rational and defined at $\nu=0$. The function $a=f \mu$ is a meromorphic function on $\mathcal{C}$, which satisfies

$$
a \circ I=-a
$$

and

$$
a^{*}=a \text {. }
$$

Proof. By the definition of $\mu^{2}$, the quotient $\frac{a^{2}}{\mu^{2}}$ is rational and has only poles and zeroes of even order. Therefore, it is the square of a rational function $f(\nu)$. Since $a^{2}$ has a zero of odd order at $\nu=0, f(\nu)$ is defined at $\nu=0$. By (4.2.2), $a=f \mu$ is a meromorphic function on $\mathcal{C}$. Since $a^{2}$ is real and non-negative on $S^{1}$, the function $a=\sqrt{a^{2}}$ takes real values over $S^{1}$ on $\mathcal{C}$, whence, by Lemma 4.3, (4.4.3) holds. Furthermore, $a \circ I=-f \mu=-a$ and (4.4.2) holds.

Lemma. Let $\phi$ be a meromorphic function on $\mathcal{C}$. Then $\phi$ is antisymmetric w.r.t. the hyperelliptic involution I, i.e. $\phi \circ I=-\phi$, iff $\phi(\nu, \mu)=f(\nu) \mu$, where $f$ is a rational function. Furthermore, in this case the following holds:

1. Locally around $P_{0}, \phi$ is an odd meromorphic function of the local coordinate $\lambda$.

2. The product $\phi$ a can be identified with a rational function of $\nu$ on $\mathbb{C P}_{1}$.

Proof. The equivalence statement follows immediately from the representation (4.2.2) of meromorphic functions on $\mathcal{C}$. Now consider $\phi_{0}=\phi \circ \lambda^{(-1)}$, where $\lambda^{(-1)}$ denotes the inverse of the local coordinate map $\lambda$ around $P_{0}$. Noting that 4.2 implies $\lambda \circ I \circ \lambda^{(-1)}(z)=-z$, we obtain $\phi_{0}(-z)=\phi \circ I \circ$ $\lambda^{(-1)}(z)=-\phi_{0}(z)$, and therefore 1 . holds.

Finally, by (4.4.2), we have that $\phi a$ is invariant under $I$, whence, by Remark 4.2 , can be identified with a meromorphic function $(\phi a)(\nu)$ on $\mathbb{C} \mathrm{P}_{1}$.

4.5. Let us also define the Riemann surface $\mathcal{C}^{\prime}$ on which $\sqrt{a^{2}(\lambda)}$ is meromorphic. To be precise (see [19, Lemma III.1.7]), let $\mathcal{C}^{\prime}$ be the hyperelliptic curve associated with the plane affine curve $\tilde{\mathcal{C}}^{\prime}$ defined by the algebraic 
equation

$$
\tilde{\mu}^{2}=\prod_{i=1}^{2 g}\left(\lambda-\sqrt{\nu_{i}}\right)\left(\lambda+\sqrt{\nu_{i}}\right) .
$$

The holomorphic function $\lambda$ extends to a holomorphic map $\pi^{\prime}: \mathcal{C}^{\prime} \rightarrow \mathbb{C P}_{1}$ of degree 2.

As for $\mathcal{C}$, we will identify the points of $\mathcal{C}^{\prime}$ which don't lie over $\lambda=\infty$ with pairs $(\lambda, \tilde{\mu})$. It should be noted, that $\mathcal{C}^{\prime}$ has no branchpoints over $\lambda=0$ and $\lambda=\infty$ since $a^{2}$ has, as an even function of $\lambda$, a zero of even order at 0 . In particular, $\left(\pi^{\prime}\right)^{-1}(\infty)$ consists of two different points $P_{\infty}^{(1)}$ and $P_{\infty}^{(2)}$. By $P_{0}^{(1)}$ and $P_{0}^{(2)}$ we will denote the two covering points of $\lambda=0$. Clearly, $\lambda$ is a local coordinate around $P_{0}^{(1)}$ and $P_{0}^{(2)}$ and $\lambda^{-1}$ is a local coordinate around $P_{\infty}^{(1)}$ and $P_{\infty}^{(2)}$.

Every meromorphic function $\tilde{f}$ on $\mathcal{C}^{\prime}$ is of the form [19, Proposition 1.10]

$$
\tilde{f}(\lambda, \tilde{\mu})=\tilde{f}_{1}(\lambda)+\tilde{f}_{2}(\lambda) \tilde{\mu}
$$

with two rational functions $\tilde{f}_{1}$ and $\tilde{f}_{2}$.

Remark 1. Let $I^{\prime}(\lambda, \tilde{\mu})=(\lambda,-\tilde{\mu})$ be the hyperelliptic involution on $\mathcal{C}^{\prime}$. As in Remark 4.2, we will use the representation (4.5.2) to identify rational functions of $\lambda$ with $I^{\prime}$-invariant meromorphic functions on $\mathcal{C}^{\prime}$. For the understanding of the rest of the paper it will be helpful to investigate the relations between $\mathcal{C}$ and $\mathcal{C}^{\prime}$. In preparation of this we introduce the notation $\mathbb{C}_{\nu}, \mathbb{C}_{\nu} \mathrm{P}_{1}, \mathbb{C}_{\nu}^{*}$ for the $\nu$-plane, the projective $\nu$-plane and $\mathbb{C}_{\nu}^{*}=\mathbb{C}_{\nu} \backslash\{0\}$, respectively. Similar notation will be used for the $\lambda$-plane. We define

$$
\rho_{0}: \mathbb{C}_{\lambda} \mathrm{P}_{1} \longrightarrow \mathbb{C}_{\nu} \mathrm{P}_{1}, \quad \rho_{0}(\lambda)=\lambda^{2} .
$$

Remark 2. 1. For similarly defined functions on $\mathbb{C}_{\lambda}$ and $\mathbb{C}_{\lambda}^{*}$ we will use the same notation $\rho_{0}$.

2. In the rest of the paper we will carefully state where functions, 1-forms etc. are defined. Notation, like $\rho_{0}$, will be used for several closely related maps, 1-forms etc.

3. If $g$ is a map defined on $\mathbb{C}_{\nu}$, then " $g$ on $\mathbb{C}_{\lambda}$ " means $g \circ \rho_{0}$. Similarly, if $\delta$ is a 1 -form on $\mathbb{C}_{\nu}, " \delta$ on $\mathbb{C}_{\lambda}$ " means $\rho_{0}^{*} \delta$, the "pullback of $\delta$ relative to $\rho_{0} "$. Similar conventions will be applied to various other maps.

Before investigating the relation between $\mathcal{C}$ and $\mathcal{C}^{\prime}$ we introduce some more notation

$$
\pi: \mathcal{C} \rightarrow \mathbb{C}_{\nu} \mathrm{P}_{1}
$$


where $\pi$ is the extension of $(\nu, \mu) \rightarrow \nu$ as stated in Theorem 4.1. Similarly we will use

$$
\pi^{\prime}: \mathcal{C}^{\prime} \rightarrow \mathbb{C}_{\lambda} \mathrm{P}_{1}
$$

Note that 0 and $\infty$ are branchpoints for $\pi$ but not for $\pi^{\prime}$.

Next we consider the map

$$
\rho: \mathbb{C}_{\lambda} \times \mathbb{C}_{\tilde{\mu}} \rightarrow \mathbb{C}_{\nu} \times \mathbb{C}_{\mu}, \quad \rho(\lambda, \tilde{\mu})=\left(\lambda^{2}, \lambda \tilde{\mu}\right) .
$$

Also recall the definition of the antiholomorphic involution $\hat{\sigma}$ on $\mathcal{C}$ given by (4.3.2). Similarly we have an antiholomorphic involution $\hat{\sigma}^{\prime}$ on $\mathcal{C}^{\prime}$ defined on $\tilde{\mathcal{C}}^{\prime} \backslash\left\{P_{0}^{(1)}, P_{0}^{(2)}\right\}$ by

$$
\hat{\sigma}^{\prime}(\lambda, \tilde{\mu})=\left(\bar{\lambda}^{-1}, \bar{\lambda}^{-2 g}\left(\prod_{j=1}^{2 g} \nu_{j}\right)^{\frac{1}{2}} \tilde{\mu}\right) .
$$

Theorem. The map $\rho$, restricted to $\tilde{\mathcal{C}}^{\prime}$, extends to a surjective and holomorphic map $\rho: \mathcal{C}^{\prime} \rightarrow \mathcal{C}$. Moreover, $\rho \circ \hat{\sigma}^{\prime}=\hat{\sigma} \circ \rho$ and $\rho \circ I^{\prime}=I \circ \rho$ holds.

Proof. Let $(\lambda, \tilde{\mu}) \in \tilde{\mathcal{C}}^{\prime}$. Then $\rho(\lambda, \tilde{\mu})=\left(\lambda^{2}, \lambda \tilde{\mu}\right)$ satisfies

$$
(\lambda \tilde{\mu})^{2}-\lambda^{2} \prod_{j=1}^{2 g}\left(\lambda^{2}-\nu_{j}\right)=\lambda^{2}\left(\tilde{\mu}^{2}-\prod_{j=1}^{2 g}\left(\lambda^{2}-\nu_{k}\right)\right)=0 .
$$

Conversely, if $(\nu, \mu) \in \tilde{\mathcal{C}}^{*}$, then $\left(\sqrt{\nu}, \frac{1}{\sqrt{\nu} \mu}\right) \in \mathbb{C}_{\lambda} \times \mathbb{C}_{\tilde{\mu}}$ satisfies

$$
\left(\frac{1}{\sqrt{\nu}}\right)^{2}-\prod_{j=1}^{2 g}\left(\sqrt{\nu}-\sqrt{n u_{j}}\right)\left(\sqrt{\nu}+\sqrt{\nu_{j}}\right)=\frac{1}{\nu} \mu^{2}-\prod_{j=1}^{2 g}\left(\nu-\nu_{j}\right)=0 .
$$

If $(\nu, \mu) \in \tilde{\mathcal{C}}, \nu=0$, then $\mu=0$ and $\rho\left(0, \tilde{\mu}_{0}\right)=(0,0)$, where $\tilde{\mu}_{0}$ is chosen such that $\left(0, \tilde{\mu}_{0}\right) \in \tilde{\mathcal{C}}^{\prime}$. Since for every point of $\tilde{\mathcal{C}}$ either $\nu$ or $\mu$ is a local coordinate, it is easy to verify that $\rho: \tilde{\mathcal{C}}^{\prime} \rightarrow \tilde{\mathcal{C}}$ is holomorphic. Finally, let $P=(\lambda(z), \tilde{\mu}(z))$ denote the points in a neighbourhood of one of the points above $\infty$ on $\mathcal{C}^{\prime}$. Then, by [19, Chapter III], this point is described in $\tilde{\mathcal{C}}^{\prime}$ by $P=\left(\lambda(z)^{-1} \tilde{\mu}(z) \lambda(z)^{-g^{\prime}-1}\right)$ where we have $4 g=2 g^{\prime}+2$, thus $g^{\prime}=2 g-1$. Then $\rho(P)=\left(\lambda(z)^{-2}, \tilde{\mu}(z) \lambda(z)^{-g^{\prime}-2}\right)$. In the chart around $P_{\infty} \in \mathcal{C}$ this is

$$
\left(\lambda(z)^{2}, \tilde{\mu}(z) \lambda(z)^{-g^{\prime}-2}\left(\lambda(z)^{-2}\right)^{-(g+1)}\right)=\left(\lambda(z)^{2}, \tilde{\mu}(z) \lambda(z)\right) .
$$


This shows, that $\rho$ extends to a surjective holomorphic map from $\mathcal{C}^{\prime}$ to $\mathcal{C}$. The last statement is a straightforward computation.

Corollary 1. The following diagram of surjective holomorphic maps is commutative.

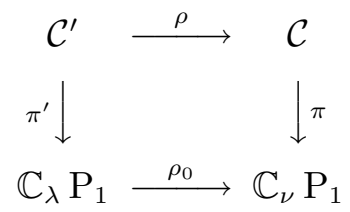

As a consequene of the Theorem above we can lift every function on $\mathcal{C}$ to a function on $\mathcal{C}^{\prime}$ via composition with $\rho$. This applies in particular to meromorphic functions. Denoting by $\mathcal{M}(\mathcal{C})$ and $\mathcal{M}\left(\mathcal{C}^{\prime}\right)$ the fields of meromorphic functions on $\mathcal{C}$ and $\mathcal{C}^{\prime}$ respectively, we obtain the:

Proposition. a) The map $\hat{\rho}: \mathcal{M}(\mathcal{C}) \rightarrow \mathcal{M}\left(\mathcal{C}^{\prime}\right), \hat{\rho}(f)=f \circ \rho$ is an injective homomorphism of fields.

b) A function $f^{\prime} \in \mathcal{M}\left(\mathcal{C}^{\prime}\right)$ is in the image of $\hat{\rho}$ iff

$$
f^{\prime}(-\lambda, \tilde{\mu})=f^{\prime}(\lambda,-\tilde{\mu}) .
$$

Proof. a) Straightforward.

b) If $f^{\prime}$ is in the image of $\hat{\rho}$, then

$$
f^{\prime}(\lambda, \tilde{\mu})=(f \circ \rho)(\lambda, \tilde{\mu})=f\left(\lambda^{2}, \lambda \tilde{\mu}\right)=f_{1}\left(\lambda^{2}\right)+f_{2}\left(\lambda^{2}\right) \lambda \tilde{\mu}
$$

where we have used (4.2.2). A comparison with (4.5.2) now shows

$$
\begin{aligned}
& f_{1}^{\prime}(\lambda)=f_{1}\left(\lambda^{2}\right) \\
& f_{2}^{\prime}(\lambda)=f_{2}\left(\lambda^{2}\right) \lambda,
\end{aligned}
$$

from which (4.5.12) follows. Conversely, assume that $f^{\prime}(\lambda, \tilde{\mu})=f_{1}^{\prime}(\lambda)+$ $f_{2}^{\prime}(\lambda) \tilde{\mu}$ satisfies (4.5.12). Then $f_{1}^{\prime}$ is even and $f_{2}^{\prime}$ is odd in $\lambda$ and we can find functions $f_{1}$ and $f_{2}$ of $\nu=\lambda^{2}$ such that (4.5.14) and (4.5.15) hold. Obviously, $f^{\prime}=f \circ \rho$ for $f=f_{1}+f_{2} \mu \in \mathcal{M}(\mathcal{C})$.

Corollary 2. The function $a^{2}(\lambda)$ on $\mathbb{C}_{\lambda}$ is the square of a meromorphic function on $\mathcal{C}^{\prime}$.

Proof. By Proposition 4.4, $a^{2}(\nu)$ is the square of a meromorphic function on $\mathcal{C}$. By the proposition above, we know that $\check{a}=a \circ \rho$ is meromorphic on 
$\mathcal{C}^{\prime}$. Moreover, $\check{a} \circ I^{\prime}=a \circ \rho \circ I^{\prime}=a \circ I \circ \rho=-a \circ \rho=-\check{a}$, where we have used (4.4.2). This implies that $(\check{a})^{2}$ descends to the meromorphic function $(\check{a})^{2}(\lambda)=a^{2}\left(\lambda^{2}\right)$ on $\mathbb{C}_{\lambda}$ by Remark 4.2.

Remark 3. 1. The diagram 4.5.11 naturally induces also diagrams, where the projective spaces are replaced by $\mathbb{C}_{\lambda}$ and $\mathbb{C}_{\nu}$ or by $\mathbb{C}_{\lambda}^{*}$ and $\mathbb{C}_{\nu}^{*}$.

2. Each of the surjective maps of any of the above diagrams induces an injective map on the level of meromorphic functions via composition.

4.6. Let us define the non-compact Riemann surfaces

$$
\mathcal{C}^{*}=\mathcal{C} \backslash\left\{P_{0}, P_{\infty}\right\} \text { and } \mathcal{C}^{\prime *}=\mathcal{C}^{\prime} \backslash\left\{P_{0}^{(1)}, P_{0}^{(2)}, P_{\infty}^{(1)}, P_{\infty}^{(2)}\right\} .
$$

We already know, that $a$ is meromorphic on $\mathcal{C}^{\prime}$ and $\mathcal{C}$, and therefore also on $\mathcal{C}^{*}$. Now we prove the following important result:

Theorem. The functions $\alpha$ and $\beta$ are holomorphic on $\mathcal{C}^{*}$. The functions $b=\sqrt{b^{2}}$ and $c=\sqrt{c^{2}}$ are meromorphic on $\mathcal{C}^{\prime}$ without poles over 0 and $\infty$.

Proof. We know by $\mathrm{a}^{\prime}$ ) and $\mathrm{d}^{\prime}$ ) in Theorem 3.7, that $\beta a, \alpha$, and $\beta^{2}$ are even functions in $\lambda$, which are defined and holomorphic on $\mathbb{C}^{*}$. Therefore, after replacing $\lambda^{2}$ by $\nu$ Remark 4.2 applies and the functions above can be identified with $I$-invariant holomorphic functions on $\mathcal{C}^{*}$. Since also $a$ is meromorphic on $\mathcal{C}$, we have that $\beta=\frac{\beta a}{a}$ is a meromorphic function on $\mathcal{C}^{*}$. Since the square $\beta^{2}(\nu)$ is holomorphic on $\mathbb{C}^{*}, \beta$ has no poles on $\mathcal{C}^{*}$, which shows that $\beta$ is holomorphic on $\mathcal{C}^{*}$.

Applying (4.5.11) we pull back $\beta$ from $\mathcal{C}^{*}$ to a holomorphic function (also denoted by $\beta$ ) on $\mathcal{C}^{\prime *}$. Furthermore, $\beta b$ and $\beta c$ are odd functions of $\lambda$, which are defined and holomorphic on $\mathbb{C}^{*}$. Thus, $\beta b, \beta c, b=\frac{\beta b}{\beta}$ and $c=\frac{\beta c}{\beta}$ are meromorphic on $\mathcal{C}^{\prime *}$. Since, by Theorem $3.7, b(\lambda)$ and $c(\lambda)$ are in $\mathcal{A}_{r}^{+}$, they can be continued holomorphically to $\lambda=0$ on $\mathbb{C P}_{1}$. By e) in Theorem 3.7, the same holds for $b$ and $c$ around $\lambda=\infty$. Since $\lambda$ is a local coordinate around $P_{0}^{(1)}$ and $P_{0}^{(2)}$ and $\lambda^{-1}$ is a local coordinate around $P_{\infty}^{(1)}$ and $P_{\infty}^{(2)}$, the functions $b$ and $c$ can be extended holomorphically to these points, which finishes the proof.

Proposition. With $(\cdot)^{*}$ defined in Section 4.3 , we have

$$
\begin{gathered}
\alpha^{*}=\alpha, \quad \beta^{*}=-\beta \quad \text { on } \mathcal{C}^{*} \text { and on } \mathcal{C}^{\prime *}, \\
b^{*}=c \text { on } \mathcal{C}^{\prime}, \\
\alpha \circ I=\alpha \text { and } \beta \circ I=-\beta \text { on } \mathcal{C}^{*},
\end{gathered}
$$




$$
\begin{gathered}
\alpha \circ I^{\prime}=\alpha \text { and } \beta \circ I^{\prime}=-\beta \text { on } \mathcal{C}^{* *}, \\
b \circ I=-b \text { and } c \circ I=-c \text { on } \mathcal{C}^{\prime} .
\end{gathered}
$$

Proof. By b') in Theorem 3.7, as functions on $\mathbb{C}^{*}, \alpha$ and $\beta^{2}$ are real on $S^{1}$, i.e., if we identify $\alpha$ and $\beta^{2}$ with holomorphic functions on $\mathcal{C}^{*}$, then they are real on $\mathcal{S}$ defined in (4.3.1). Thus, by Lemma 4.3, $\alpha^{*}=\alpha$ holds. By $\left.c^{\prime}\right)$ in Theorem 3.7, $\beta^{2}$ is non-positive on $S^{1}$. Therefore, the holomorphic function $\beta$ on $\mathcal{C}^{*}$ is on $\mathcal{S}$ the square root of a non-positive real function. Hence, $\beta$ is purely imaginary on $\mathcal{S}$. This implies $\beta^{*}=-\beta$, by Lemma 4.3. The statement on $\mathcal{C}^{\prime *}$ follows from this since $\rho$ intertwines $\hat{\sigma}$ with $\hat{\sigma}^{\prime}$ by Theorem 4.5. To verify (4.6.2) we use again the representation $b=\frac{\beta b}{\beta}$ on $\mathcal{C}^{\prime *}$ and (3.5.12) and obtain $b^{*}=\frac{(\beta b)^{*}}{\beta^{*}}=\frac{\beta c}{\beta}=c$. The first relation in (4.6.3) is clear, since $\alpha$ is defined on $\mathbb{C}_{\nu}^{*}$. For the second we use $\beta=\frac{\beta a}{a}$ and obtain $\beta \circ I=\frac{(\beta a) \circ I}{a \circ I}=-\frac{\beta a}{a}=-\beta$, since $\beta a$ is defined on $\mathbb{C}_{\nu}^{*}$ and (4.4.2) holds. (4.6.4) follows from (4.6.3) since $\rho$ intertwines $I$ with $I^{\prime}$. Finally, $b \circ I=\frac{(\beta b) \circ I}{\beta \circ I}=-\frac{\beta b}{\beta}=-b$. and similarly $c \circ I=-c$.

4.7. Let us pause to collect some of the implications of Theorem 4.6. By (3.3.2), the matrix $S=h_{+} A h_{+}^{-1}=\left(\begin{array}{cc}a & b \\ c & -a\end{array}\right)$ can be written as

$$
S=h_{+} D^{-1}\left(\begin{array}{cc}
1 & 0 \\
0 & -1
\end{array}\right) D h_{+}^{-1} .
$$

The entries of $S$ are all meromorphic functions on $\mathcal{C}^{\prime}$. By linear algebra over the field $\mathcal{M}\left(\mathcal{C}^{\prime}\right)$ of meromorphic functions on $\mathcal{C}^{\prime}$, we get that there exist nonzero elements $x_{ \pm}$of $\mathcal{M}\left(\mathcal{C}^{\prime}\right)^{2}$, written as column vectors, such that $S x_{ \pm}=$ $\pm x_{ \pm}$. These vectors are unique up to multiplication by elements of $\mathcal{M}\left(\mathcal{C}^{\prime}\right)$. They define a two-dimensional vector-bundle over $\mathcal{C}^{\prime}$, the "eigenbundle" of $S$ over $\mathcal{C}^{\prime}$. If we fix them by requiring that

$$
\left(x_{+}(\lambda), x_{-}(\lambda)\right)=h_{+}(\lambda) D^{-1} \quad \text { for }|\lambda| \leq r,
$$

then they define a lift of $h_{+}$to a matrix function with entries meromorphic on $\mathcal{C}^{\prime}$.

Since, by (3.4.7) and (3.3.10), the matrix $\chi=\alpha I+\beta h_{+} A h_{+}^{-1}$ can be written in the form

$$
\chi=h_{+} D^{-1}\left(\begin{array}{cc}
\alpha+\beta & 0 \\
0 & \alpha-\beta
\end{array}\right) D h_{+}^{-1},
$$


we see, that the branchpoints of $\mathcal{C}^{\prime}$ are points, where the eigenvalues of $\chi$ coincide, i.e., where $\chi$ fails to be regular semisimple.

4.8. The next two sections are a digression on finite type surfaces.

Let $\Psi: \mathbb{C} \rightarrow \mathbb{R}^{3}$ be a CMC-immersion with extended frame $F(z, \lambda)$ which is generated by $r$-dressing the cylinder with $h_{+} \in \Lambda_{r}^{+} \mathbf{S L}(2, \mathbb{C})_{\sigma}$. Let $\mathcal{Z}$ be the abelian Lie-subalgebra of all $\zeta \in \Lambda_{r} \operatorname{sl}(2, \mathbb{C})_{\sigma}$, such that

- $[\zeta(\lambda), A]=0$ for all $\lambda \in C_{r}$ and

- $\zeta$ can be extended to a meromorphic function on $I^{(r)}$ which has precisely one pole at $\lambda=0$.

Remark 1. By the discussion in Section 3.3 and the tracelessness of the elements of $\Lambda_{r} \mathbf{s l}(2, \mathbb{C})_{\sigma}$, we can write each $\zeta \in \mathcal{Z}$ uniquely as

$$
\zeta(\lambda)=\phi(\lambda) A
$$

where $\phi \in \mathcal{A}_{r}$ is odd in $\lambda$, and has precisely one pole at $\lambda=0$.

Let $F: \mathbb{C} \rightarrow \Lambda_{r} \mathbf{S U}(2)_{\sigma}$ be an extended frame in the $r$-dressing orbit of the cylinder. Thus, there is $h_{+} \in \Lambda_{r}^{+} \mathbf{S L}(2, \mathbb{C})_{\sigma}$ and $p_{+}: \mathbb{C} \rightarrow \Lambda_{r}^{+} \mathbf{S L}(2, \mathbb{C})_{\sigma}$, such that

$$
F=h_{+} e^{\left(\lambda^{-1} z-\lambda \bar{z}\right) A} p_{+}(z, \lambda)^{-1} .
$$

For each $\zeta \in \mathcal{Z}$ we define

$$
h_{+} \# \zeta=U^{-1} h_{+} e^{\zeta(\lambda)},
$$

where $U \in \Lambda_{r} \mathbf{S U}(2)_{\sigma}$ is determined by the Iwasawa decomposition (2.2.7), such that $h_{+} \# \zeta \in \Lambda_{r}^{+} \mathbf{S L}(2, \mathbb{C})_{\sigma}$. The action of $\zeta$ on $h_{+}$descends via (3.1.1) to an action on the frame $F$ :

$$
F \# \zeta=\left(h_{+} \# \zeta\right) e^{\left(\lambda^{-1} z-\lambda \bar{z}\right) A} \tilde{p}_{+}(z, \lambda)^{-1}=U^{-1} h_{+} e^{\left(\lambda^{-1} z-\lambda \bar{z}\right) A+\zeta(\lambda)} \tilde{p}_{+}(z, \lambda)^{-1},
$$

where $\tilde{p}_{+}: \mathbb{C} \rightarrow \Lambda_{r}^{+} \mathbf{S L}(2, \mathbb{C})_{\sigma}$ is chosen such that $F \# \zeta \in \Lambda_{r} \mathbf{S U}(2)_{\sigma}$ and $(F \# \zeta)(0, \lambda)=I$.

Our definition of $\mathcal{Z}$ and the associated action on the $r$-dressing orbit of the standard cylinder are obtained from the definitions in [2, Section 4] for the special case of harmonic maps into $\mathbf{S U}(2) / \mathbf{U}(1)$. By [2, Prop. 4.1], we have:

Lemma. 1. Eqs. (4.8.3) and (4.8.4) define an action of $\mathcal{Z}$ on the $r$-dressing orbit of the cylinder which is compatible with the linear structure of $\mathcal{Z}$. In particular for $\zeta, \zeta^{\prime} \in \mathcal{Z}$,

$$
F \#\left(\zeta+\zeta^{\prime}\right)=(F \# \zeta) \# \zeta^{\prime}=\left(F \# \zeta^{\prime}\right) \# \zeta .
$$


2. If $\zeta, \zeta^{\prime} \in \mathcal{Z}$, such that $\zeta-\zeta^{\prime}$ can be extended holomorphically to $I^{(r)}$, then $F \# \zeta=F \# \zeta^{\prime}$.

In other words, each $\zeta \in \mathcal{Z}$ generates a "higher flow" on the set of CMCimmersions in the $r$-dressing orbit of the cylinder by

$$
F(z, t, \lambda)=F(z, \lambda) \#(t \zeta), \quad t \in \mathbb{R}
$$

For each $t \in \mathbb{R}$, Sym's formula gives a new associated family of CMCimmersions with extended frame $F(z, t, \lambda)$. These higher flows commute by (4.8.5).

Remark 2. 1. The extended frames $F \# \zeta$ and $F(z, t, \lambda)$ all satisfy the normalization condition (2.1.17).

2. Using (2.1.13)-(2.1.16) and the equivalence of (2.1.12) and (2.1.6), (2.1.7), we see, that (4.8.3), (4.8.4) and (4.8.6) also defines an action on the set of real valued solutions $u(z, \bar{z})$ of (2.1.6). Since $E \equiv 1$ for all surfaces in the $r$-dressing orbit of the cylinder, we get by setting $H=-2$, that this defines a hierarchy of commuting flows of the integrable sinh-Gordon equation.

4.9. We say, that the flow defined by $\zeta \in \mathcal{Z}$ acts trivial on an extended frame $F$ in the dressing orbit of the cylinder, iff

$$
F(z, t, \lambda)=U_{0}(t) F(z, \lambda) U_{0}(t)^{-1} \quad \text { for all } t \in \mathbb{R},
$$

where $U_{0}(t) \in \mathbf{U}(1)$ is a $\lambda$-independent unitary matrix. By [4], Corollary 4.1, this is equivalent to the fact that the associated families $\Psi(z, t, \lambda)$ and $\Psi(z, \lambda)$ defined by $F(z, t, \lambda)$ and $F(z, \lambda)=F(z, 0, \lambda)$, differ only by a proper Euclidean motion in $\mathbb{R}^{3}$. Clearly, the set $\mathcal{Z}^{\prime} \subset \mathcal{Z}$ of elements which generate trivial flows, is a linear subspace of $\mathcal{Z}$.

Definition. A CMC-surface in the $r$-dressing orbit of the cylinder is of finite type iff the subspace $\mathcal{Z}^{\prime}$ of trivial flows has finite codimension in $\mathcal{Z}$.

Let $\zeta \in \mathcal{Z}^{\prime}$. Then, by [4, Corollary 2.6], all surfaces generated by the $\zeta$-flow have the same metric. Therefore, using Remark 2 in Section 4.8, we see, that $\zeta$ generates a trivial flow of the sinh-Gordon equation, i.e., $u(z, \bar{z}, t)=u(z, \bar{z})$ for all $t \in \mathbb{R}$. In [2, Theorem 4.2] it was shown, that the conformal factor $u(z, \bar{z})$ associated to a CMC-immersion $\Psi$ is a finite type solution of the sinh-Gordon-equation (for a definition see [20] or [7]) iff $\Psi$ is of finite type in the sense of Definition 4.9.

In [20] it was shown, that for a surface with doubly periodic metric, in particular for CMC-tori, the conformal factor $u$ is a finite type solution of the sinh-Gordon equation. For more general CMC-surfaces with periodic metric, 
e.g., for CMC-surfaces which are topological cylinders, this is in general not true. However, for surfaces in the $r$-dressing orbit of the cylinder, we have the remarkable:

Theorem. Every CMC-surface with periodic metric in the $r$-dressing orbit of the cylinder is of finite type.

Proof. Consider a CMC-surface $\Psi: \mathbb{C} \rightarrow \mathbb{R}^{3}$ with periodic metric defined by $r$-dressing the cylinder with $h_{+} \in \Lambda_{r}^{+} \mathbf{S L}(2, \mathbb{C})_{\sigma}$ and the hyperelliptic curve $\mathcal{C}$ defined in Section 4.1. Then, by Proposition 4.4, the diagonal entries of $S=h_{+} A h_{+}^{-1}$ are meromorphic on $\mathcal{C}$.

For arbitrary $\zeta \in \mathcal{Z}$ we write $\zeta=\hat{\phi}(\lambda) A$, where $\hat{\phi}$ is odd and meromorphic on $I^{(r)}$ with a pole only at $\lambda=0$. If $\hat{\phi}(\lambda)$ and $\hat{\phi}^{\prime}(\lambda)$ have the same principal part at $\lambda=0$, then for $\zeta=\hat{\phi}(\lambda) A$ and $\zeta^{\prime}=\hat{\phi}^{\prime}(\lambda) A, \zeta-\zeta^{\prime}$ is holomorphic on $I^{(r)}$. Therefore, by Lemma $4.8, \zeta$ and $\zeta^{\prime}$ generate the same flow. Thus, to show that $\Psi$ is of finite type, it is enough to construct a trivial flow for all but a finite dimensional space of principal parts of $\hat{\phi}$.

If $N \geq g+1$, then the function

$$
\tilde{\phi}_{N}(\nu, \mu)=\nu^{-N} \mu
$$

is by (4.2.2) meromorphic on $\mathcal{C}$, has only a pole of order $2 N-1$ at $P_{0}$ and is otherwise holomorphic. Obviously,

$$
\tilde{\phi}_{N} \circ I=-\tilde{\phi}_{N}
$$

We define

$$
\phi_{N}=\tilde{\phi}_{N}-\tilde{\phi}_{N}^{*}
$$

where $(\cdot)^{*}$ was defined in Section 4.3. Then $\phi_{N}$ is a meromorphic function on $\mathcal{C}$ with a pole of order $2 N-1$ at $P_{0}$ and $P_{\infty}$, which is holomorphic on $\mathcal{C}^{*}$ and satisfies

$$
\phi_{N} \circ I=-\phi_{N}
$$

and

$$
\phi_{N}^{*}=-\phi_{N},
$$

since $(\cdot)^{*}$ is an involution which, by Proposition 4.3, commutes with $I$. By (4.9.5) and Lemma 4.4,

$\phi_{N} a$ is a rational function on $\mathbb{C}_{\nu} \mathrm{P}_{1}$ and an even rational function on $\mathbb{C}_{\lambda} \mathrm{P}_{1}$. 
By (4.9.6) and Proposition 4.4, $\phi_{N} a$ satisfies

$$
\left(\phi_{N} a\right)^{*}=-\phi_{N} a \text { on } \mathbb{C}_{\nu} \mathrm{P}_{1} \text { and on } \mathbb{C}_{\lambda} \mathrm{P}_{1} \text {. }
$$

By the arguments above, Proposition 4.4 and Theorem 4.6, the functions $\Phi_{N}, a, b, c, \alpha$ and $\beta$ an all be considered being defined on $\mathcal{C}^{\prime *}$. By writing $\phi_{N} b=\frac{\beta b}{\beta a}\left(\phi_{N} a\right), \phi_{N} c=\frac{\beta c}{\beta a}\left(\phi_{N} a\right)$, using (4.9.7) and b) and d') of Theorem 3.7, we get that $\phi_{N} b$ and $\phi_{N} c$ are odd meromorphic functions on $\mathbb{C}_{\lambda}^{*}$. By writing $\phi_{N} b=\frac{b}{a}\left(\phi_{N} a\right), \phi_{N} c=\frac{c}{a}\left(\phi_{N} a\right)$, we see that $\phi_{N} b$ and $\phi_{N} c$ can be extended meromorphically to $\lambda=0$. Furthermore, by (3.5.12), (4.9.8) and Proposition 4.6 we have

$$
\left(\phi_{N} c\right)^{*}=\frac{(\beta c)^{*}}{(\beta a)^{*}}\left(\phi_{N} a\right)^{*}=-\frac{-\beta b}{-\beta a}\left(\phi_{N} a\right)=-\phi_{N} b .
$$

In particular, $\phi_{N} b$ and $\phi_{N} c$ can be extended meromorphically to $\lambda=\infty$, whence

$$
\phi_{N} b \text { and } \phi_{N} c \text { are odd rational functions on } \mathbb{C}_{\lambda} \mathrm{P}_{1} \text {. }
$$

Since $a^{2}, b^{2}$ and $c^{2}$ are rational functions of $\nu$, we can choose a polynomial $\tilde{\varphi}(\nu), \tilde{\varphi}(0) \neq 0$, such that $\tilde{\varphi}^{2} a^{2}, \tilde{\varphi}^{2} b^{2}$ and $\tilde{\varphi}^{2} c^{2}$ are holomorphic on $\mathbb{C}_{\nu}^{*}$. Then $\tilde{\varphi}^{*}$ is a rational function with a pole only at $\nu=0$. If we define

$$
\varphi=\tilde{\varphi} \tilde{\varphi}^{*}
$$

then the function $\varphi(\nu)$ is rational, real on $S^{1}$ and $\varphi, \varphi^{2} a^{2}, \varphi^{2} b^{2}$ and $\varphi^{2} c^{2}$ have no poles on $\mathbb{C}_{\nu}^{*}$. We set $\hat{\phi}_{N}=\varphi \phi_{N}$ on $\mathcal{C}^{*}$. Then, since $\phi_{N}$ has no poles on $\mathcal{C}^{*},(4.9 .7)$ and $(4.9 .10)$ yield

$$
\hat{\phi}_{N} a \text { is an even rational function of } \lambda \text {, which is holomorphic on } \mathbb{C}^{*} \text {, }
$$

$\hat{\phi}_{N} b$ and $\hat{\phi}_{N} c$ are odd rational functions of $\lambda$, which are holomorphic on $\mathbb{C}^{*}$.

Furthermore, since $\varphi$ is real on $S^{1},(4.9 .8)$ and (4.9.9) give

$$
\left(\hat{\phi}_{N} a\right)^{*}=-\hat{\phi}_{N} a, \quad \hat{\phi}_{N} c=-\left(\hat{\phi}_{N} b\right)^{*} \quad \text { on } \mathbb{C}_{\nu}^{*} \text { and on } \mathbb{C}_{\lambda}^{*} .
$$

By (4.9.12) and (4.9.13), the matrix function

$$
\left(\hat{\phi}_{N} S\right)(\lambda)=\left(\begin{array}{cc}
\hat{\phi}_{N} a & \hat{\phi}_{N} b \\
\hat{\phi}_{N} c & -\hat{\phi}_{N} a
\end{array}\right)
$$


is rational and holomorphic on $\mathbb{C}_{\lambda}^{*}$ and satisfies the twisting condition (2.2.1). In addition, by $(4.9 .14),(\hat{\phi} S)^{*}=-\hat{\phi} S$, i.e.,

$\hat{\phi}_{N} S$ is in the Lie algebra $\Lambda_{r} \mathbf{s u}(2)_{\sigma}$ and extends holomorphically to $\mathbb{C}^{*}$.

The functions $\tilde{\varphi}$ and $\varphi$ constructed above are independent of $N$. As in Remark 4.2 , we identify $\varphi$ with an $I$-invariant meromorphic function on $\mathcal{C}$. Let $\kappa \in \mathbb{N}$ be the degree of the polynomial $\tilde{\varphi}(\nu)$. Then, since $\varphi(0) \neq 0, \varphi$ has a pole of even order $2 \kappa$, at $P_{0}$. We can choose the polynomial $\tilde{\varphi}$ such that $\kappa$ is minimal.

The function $\hat{\phi}_{N} a$ is rational and even in $\lambda$ and has no poles on $\mathbb{C}^{*}$. Since, by Proposition 4.4 and Lemma 4.4, $a(\lambda) \in \mathcal{A}_{r}^{+}$is odd, we get that $\hat{\phi}_{N}(\lambda)=$ $\frac{\hat{\phi}_{N} a}{a}$ is an odd meromorphic function on $I^{(r)}$, which, since $\phi_{N}$ is holomorphic on $\mathcal{C}^{\prime *}$, has only a pole at $\lambda=0$. Therefore, by Remark 1 in Section 4.8, $\zeta=\hat{\phi}_{N} A \in \mathcal{Z}$ defines a higher flow acting on the periodic surface $\Psi$. The pole of $\hat{\phi}_{N}(\lambda)$ at $\lambda=0$ is of degree $2(\kappa+N)-1$. Since $\kappa<\infty$ is independent of $N$ and since $N$ can take all but a finite number of integer values, it only remains to show, that each of the $\hat{\phi}_{N}$ generates a trivial flow.

We have

$$
h_{+} e^{t \hat{\phi}_{N} A}=h_{+} e^{t \hat{\phi}_{N} A} h_{+}^{-1} h_{+}=e^{t \hat{\phi}_{N} S} h_{+}
$$

By (4.9.16), for all $t \in \mathbb{R}$, the matrix $U(t, \lambda)=e^{t \hat{\phi}_{N} S}$ is in the twisted loop group $\Lambda_{r} \mathbf{S U}(2)_{\sigma}$ and can be extended holomorphically to $\lambda \in \mathbb{C}^{*}$. This yields $h_{+} \#\left(t \hat{\phi}_{N} A\right)=U_{0}(t) h_{+}$with some unitary $\lambda$-independent matrix $U_{0}(t)$, by the uniqueness of the Iwasawa decomposition. Thus, by (4.8.4) and (2.1.17), $F(z, t, \lambda)=U_{0}(t) F(z, \lambda) U_{0}(t)^{-1}$, and the flow generated by $\zeta_{N}=\hat{\phi}_{N} A$ is trivial. This shows, that $\mathcal{Z}^{\prime}$ has at most codimension $\kappa+g<\infty$ in $\mathcal{Z}$. Thus, $\Psi$ is a finite type surface.

Remark. Theorem 4.9 can also be obtained from more general results for integrable systems. We want to outline, how this works:

By $\mathcal{G}_{r}, 0<r<1$, we denote the group of maps from $C_{r}$ to $\mathbf{S L}(2, \mathbb{C})$ which satisfy (2.2.1) and can be continued to holomorphic functions on $I^{(r)} \backslash\{0\}$. For $0<r^{\prime} \leq r<1$, let $\rho_{r^{\prime}}^{r}: \mathcal{G}_{r} \mapsto \mathcal{G}_{r^{\prime}}$ be the restriction of maps. Using these homomorphisms of Lie groups, define $\mathcal{G}_{0}$ as the direct limit of the groups $\mathcal{G}_{r}$ for $r \rightarrow 0$. Let $\mathcal{G}_{0}^{+} \subset \mathcal{G}_{0}$ be the subgroup of maps which extend holomorphically to $\lambda=0$ and let $\mathcal{G}_{0}^{-}$be the subgroup of maps which can be continued holomorphically to $\lambda=\infty$ and take the value $I \in \mathbf{S L}(2, \mathbb{C})$ there. Clearly, $\mathcal{G}_{0}^{+} \cap \mathcal{G}_{0}^{-}=\{I\}$. As was proved in [14, Section 2.2], the multiplication map defines a diffeomorphism of $\mathcal{G}_{0}^{-} \times \mathcal{G}_{0}^{+}$onto an open, dense 
subset of $\mathcal{G}_{0}$. By arguments similar to those in [18] one can show, that $\mathcal{G}_{0}$ admits a second splitting: Let $\mathcal{G}_{0}^{r} \subset \mathcal{G}_{0}$ be the subgroup of maps which can be continued holomorphically to $\mathbb{C}^{*}$ and take values in $\mathbf{S U}(2)$ on $S^{1}$. Then multiplication $\mathcal{G}_{0}^{r} \times \mathcal{G}_{0}^{+} \rightarrow \mathcal{G}_{0}$ is a diffeomorphism onto. Since the extended frames are, by Lemma 2.2, all in $\mathcal{G}_{0}^{r}$ and since the dressing action depends only on the equivalence class of $h_{+}$in $\mathcal{G}_{0}^{+}$, we can use the group $\mathcal{G}_{0}$ with the splittings above, instead of $\Lambda_{r} \mathbf{S L}(2, \mathbb{C})_{\sigma}, 0<r<1$, to construct all CMCimmersions in the $r$-dressing orbit of the cylinder. Also, the generators $\zeta$ of the higher flows in Section 4.8 can, by the second statement in Lemma 4.8, w.l.o.g. be chosen such that $e^{t \zeta} \in \mathcal{G}_{0}^{-}$for all $t \in \mathbb{R}$. Finally, to merge both splittings into one, we can use the classical double construction presented in [13, Kapitel 4.2] (compare also the analogous construction for the groups $\Lambda_{r} \mathbf{S L}(2, \mathbb{C})_{\sigma}$ presented in $\left.[7,18,2,5]\right)$. From this, the group $\mathcal{G}_{0}$ can be recovered by a real reduction similar to the one used in [7] and [2]. With these settings, our description of solutions of the sinh-Gordon equation fits into the framework of [14] and Theorem 4.9 can be obtained from [14, Theorem 4.16].

However, for the reader's convenience, we have presented above a direct proof of Theorem 4.9 .

\section{Algebro-geometric description of surfaces with periodic metric.}

For a CMC-immersion with periodic metric, we defined in Section 4.1 a nonsingular hyperelliptic curve $\mathcal{C}$. In this section, we will show, that $\mathcal{C}$ allows us to express the periodicity conditions for CMC-immersions stated in Theorem 3.7 and Theorem 3.8 in terms of algebro-geometric data.

If the CMC-immersion $\Psi$ under consideration does not only have a periodic or doubly periodic metric, but even a compact image in $\mathbb{R}^{3}$, i.e., if $\Psi$ is a CMC-torus, then we will reproduce the classification of CMC-tori in terms of algebro-geometric data as given in [20] and [1].

We would like to point out that this classification refers to the generic case. As mentioned in the introduction, a discussion of the singular tori would be very interesting.

5.1. We will first reformulate the statement of Theorem 3.7 in terms of algebro-geometric data. We start with the same assumptions as in Section 4: Let $\Psi: \mathbb{C} \rightarrow \mathbb{R}^{3}$ be a CMC-immersion, such that $\operatorname{Sym}(\Psi)$ contains a nontrivial element $q \in \mathbb{C}^{*}$. Then we define the hyperelliptic curve $\mathcal{C}$ as in Section 4.1.

We introduce a standard homotopy basis for $\mathcal{C}$ which is adapted to the $\hat{\sigma}$-symmetry of $\mathcal{C}$ stated in Proposition 4.3 . Let $a_{1}, \ldots, a_{g}, b_{1}, \ldots, b_{g}, g$ the genus of $\mathcal{C}$, be a canonical basis of $H_{1}(\mathcal{C}, \mathbb{Z})$, such that the intersection num- 
bers are given by

(5.1.1) $a_{i} a_{j}=0$,

$$
b_{i} b_{j}=0, \quad a_{i} b_{j}=\delta_{i j}, \quad i, j=1, \ldots, g .
$$

For the cycles $a_{k}$ we choose (see [10, VII.1.1], where $a_{j} \leftrightarrow b_{j}$ compared to our conventions)

$$
a_{k}=\gamma_{k}-I \circ \gamma_{k}
$$

where $\gamma_{k}$ is a curve joining the branchpoints over $\nu_{2 k-1}$ and $\nu_{2 k}$, which satisfies $\hat{\sigma} \circ \gamma=-\gamma$. Then

$$
I \circ a_{k}=-a_{k}
$$

and

$$
\hat{\sigma} \circ a_{k}=-a_{k},
$$

since $\hat{\sigma}$ and $I$ commute. I.e., the cycles $a_{k}$ are up to orientation invariant under $\hat{\sigma}$ and $I$. In addition, we can choose $b_{k}$ such that

$$
\hat{\sigma} \circ b_{k}=b_{k}-a_{k}+\sum_{j=1}^{g} a_{j} \text {. }
$$

5.2. Now we investigate the function $p \in \mathcal{A}_{r}$ defined in (3.4.10). It is an odd meromorphic function on $I^{(r)} \subset \mathbb{C}_{\lambda}$. In general, $p$ cannot be continued to a meromorphic function on $\mathcal{C}^{*}$. However, the differential $\mathrm{d} p=p^{\prime} \mathrm{d} \lambda$ can. For this we note that $\alpha=\cosh (p)$ and $\beta=\sinh (p)$ on $I^{(r)} \backslash\{0\} \subset \mathbb{C}_{\lambda}$ by Theorem 3.7. Then, since $\alpha^{2}-\beta^{2}=1, \mathrm{~d} p=\alpha \mathrm{d} \beta-\beta \mathrm{d} \alpha$.

Let $\alpha$ and $\beta$ be the holomorphic functions on $\mathcal{C}^{*}$ defined in Theorem 4.6. Then we define the 1-form

$$
\omega=\alpha \mathrm{d} \beta-\beta \mathrm{d} \alpha \text { on } \mathcal{C}^{*} .
$$

Clearly,

$$
\omega \text { is a holomorphic } 1 \text {-form on } \mathcal{C}^{*} \text {. }
$$

Lemma. a) In the local coordinate $\lambda=\lambda(\nu, \mu)$ around $P_{0}$ we have $\omega=$ $p^{\prime}(\lambda) \mathrm{d} \lambda$.

b) $\rho^{*} \mathrm{~d} \lambda=\pi^{\prime *} \mathrm{~d} p$, where $\mathrm{d} p$ is defined on $\mathbb{C}_{\lambda}^{*}$.

Proof. a) The local coordinate $\lambda(\nu, \mu)$ around $P_{0} \in \mathcal{C}$ is the inverse map of

$$
\lambda \rightarrow(\lambda, \tilde{\mu}(\lambda)) \rightarrow\left(\lambda^{2}, \lambda \tilde{\mu}(\lambda)\right),
$$


where $\tilde{\mu}(\lambda)=\left(\prod_{j=1}^{2 g}\left(\lambda-\sqrt{\nu_{j}}\right)\left(\lambda+\sqrt{\nu_{j}}\right)\right)^{\frac{1}{2}}$. Therefore, a) follows from b). But $\rho^{*} \omega=\alpha(\lambda) \mathrm{d}(\beta \circ \rho)-(\beta \circ \rho) \mathrm{d} \alpha(\lambda)$ and $\beta \circ \rho=\frac{(\beta a) \circ \rho}{a \circ \rho}=\frac{(\beta a)(\lambda)}{a(\lambda)}$, where we have used the definition of $\beta$ in Theorem 4.6. For small $\lambda$, however, $(\beta a)(\lambda)=\beta(\lambda) a(\lambda)$, whence $\beta \circ \rho=\beta(\lambda)$ and $\rho^{*} \omega=p^{\prime}(\lambda) \mathrm{d} \lambda$ follows. Since in the coordinates under consideration, $\pi^{\prime}(\lambda, \tilde{\mu})=\lambda,\left(\pi^{\prime}\right)^{*} \mathrm{~d} p=\mathrm{d} p(\lambda)=$ $p^{\prime}(\lambda) \mathrm{d} \lambda$.

Theorem. The differential $\omega$ is a meromorphic Abelian differential of the second kind on $\mathcal{C}$ which is holomorphic on $\mathcal{C}^{*}$. It has poles of second order at $P_{0}$ and $P_{\infty}$. In local coordinates around $P_{0}$ and $P_{\infty}$ it is of the form

$$
\omega=-q \lambda^{-2} \mathrm{~d} \lambda+s_{+}(\lambda), s_{+} \text {locally holomorphic around } P_{0},
$$

$$
\omega=\bar{q}\left(\lambda^{-1}\right)^{-2} \mathrm{~d}\left(\lambda^{-1}\right)+s_{-}(\lambda), s_{-} \text {locally holomorphic around } P_{\infty} .
$$

Furthermore,

$$
\int_{a_{k}} \omega=0, \quad k=1, \ldots, g .
$$

The differential $\omega$ is uniquely determined by these properties. In addition, we have

$$
\hat{\sigma}^{*} \omega=-\bar{\omega} .
$$

Proof. From (5.2.2) we know that $\omega$ is a holomorphic 1-form on $\mathcal{C}^{*}$. The local description of $\omega$ at $P_{0}$ shows that $\omega$ has a meromorphic extension to $P_{0}$.

By Proposition 4.6 and (4.3.3), $\alpha \circ \hat{\sigma}=\bar{\alpha}$ and $\beta \circ \hat{\sigma}=-\bar{\beta}$. This yields $\hat{\sigma}^{*} \mathrm{~d} \alpha=\overline{\mathrm{d} \alpha}, \hat{\sigma}^{*} \mathrm{~d} \beta=-\overline{\mathrm{d} \beta}$. Equation (5.2.7) now follows from (5.2.1). Since $\omega$ is meromorphic around $P_{0}$ it can by $(5.2 .7)$ also be continued meromorphically to $P_{\infty}=\hat{\sigma}\left(P_{0}\right)$. This shows, that $\omega$ can be extended to an Abelian differential on $\mathcal{C}$.

Locally around $P_{0}, \lambda$ is a local coordinate on $\mathcal{C}$. We have, by (3.4.10), that

$$
\omega(\lambda)=\frac{d p(\lambda)}{d \lambda} \mathrm{d} \lambda=\left(-\lambda^{-2} q-\bar{q}+\frac{d f_{+}(\lambda)}{d \lambda}\right) \mathrm{d} \lambda,
$$


where $f_{+}$and therefore also $s_{+}=\left(-\bar{q}+\frac{d f_{+}(\lambda)}{d \lambda}\right) \mathrm{d} \lambda$ is defined and holomorphic around $\lambda=0$. This proves (5.2.4). Locally around $P_{\infty},(5.2 .4)$ and (5.2.7) yield

$$
\omega=-\overline{\hat{\sigma}^{*} \omega}=-\overline{\hat{\sigma}^{*}\left(-q \lambda^{-2} \mathrm{~d} \lambda+s_{+}\right)}=\bar{q} \lambda^{2} \mathrm{~d}\left(\lambda^{-1}\right)+s_{-}
$$

where $s_{-}=-\overline{\hat{\sigma}^{*} s_{+}}$is locally defined and holomorphic around $P_{\infty}$. This proves (5.2.5). Since $\lambda^{-1}$ is a local coordinate around $P_{\infty}$ on $\mathcal{C}$, this also shows, that $\omega$ has poles of second order at $P_{\infty}$ and $P_{0}$, and that the residue of $\omega$ vanishes at either singularity, whence it is an Abelian differential of the second kind. As such, it is by [10, Prop. III.3.3] uniquely determined by $(5.2 .4)-(5.2 .6)$.

To finish the proof, it therefore suffices to verify (5.2.6). To this end we rewrite (5.2.1) using $\alpha^{2}-\beta^{2}=1$ as

$$
\omega=\frac{\mathrm{d}(\alpha+\beta)}{\alpha+\beta}=\mathrm{d}(\ln (\alpha+\beta)) \text { on } \mathcal{C}^{*} .
$$

Since $\alpha+\beta$ is a holomorphic function without zeroes on $\mathcal{C}^{*}$, we thus obtain that the integral of $\omega$ over a closed cycle on $\mathcal{C}^{*}$ is always an integer multiple of $2 \pi i$. In particular, each such integral is purely imaginary or zero. By (5.1.4) and (5.2.7), we have

$$
\int_{a_{k}} \omega=-\int_{\hat{\sigma}_{*} a_{k}} \omega=-\int_{a_{k}} \hat{\sigma}^{*} \omega=\int_{a_{k}} \bar{\omega}=\overline{\int_{a_{k}} \omega}
$$

Thus, the integral over $a_{k}$ is real. This is only possible if the integral vanishes.

Let $\Omega_{1}$ be an Abelian differential of the second kind on $\mathcal{C}$ which is in local coordinates around $P_{\infty}$ given by

$$
\Omega_{1}=-\left(\lambda^{-1}\right)^{-2} \mathrm{~d}\left(\lambda^{-1}\right)+\text { a locally holomorphic differential around } P_{\infty}
$$

and is holomorphic on $\mathcal{C} \backslash\left\{P_{\infty}\right\}$. Such a differential exists by [10], Theorem II.5.1]. It is unique up to addition of a holomorphic differential on $\mathcal{C}$. Thus, see [10, Corollary III.3.4], if we normalize $\Omega_{1}$ by

$$
\int_{a_{k}} \Omega_{1}=0, \quad k=1, \ldots, g
$$

then $\Omega_{1}$ is determined uniquely by (5.2.12) and (5.2.13). By (5.1.3), the differential

$$
\Omega_{2}=\overline{\hat{\sigma}^{*} \Omega_{1}}
$$


has also vanishing $a$-periods and a single pole at $P_{0}$. It is given in local coordinates around $P_{0}$ by

$$
\Omega_{2}=-\lambda^{-2} \mathrm{~d} \lambda+\text { a locally holomorphic differential around } P_{0} \text {. }
$$

Since Abelian differentials are uniquely determined by their $a$-periods and the principal parts at the singularities [10, Prop. III.3.3], we have

$$
\omega=-\bar{q} \Omega_{1}+q \Omega_{2}=-\bar{q} \Omega_{1}+q \overline{\hat{\sigma}^{*} \Omega_{1}} .
$$

In addition $I^{*} \Omega_{1}$ and $-\Omega_{1}$ have the same pole divisors and both satisfy (5.2.13) and (5.2.12). Therefore,

$$
I^{*} \Omega_{1}=-\Omega_{1} .
$$

Let us define

$$
U_{k}=\int_{b_{k}} \Omega_{1}, \quad V_{k}=\int_{b_{k}} \Omega_{2} .
$$

From (5.1.5) it follows, that

$$
V_{k}=\int_{b_{k}} \Omega_{2}=\int_{b_{k}} \overline{\hat{\sigma}^{*} \Omega_{1}}=\int_{\hat{\sigma}_{*} b_{k}} \overline{\Omega_{1}}=\overline{\int_{b_{k}} \Omega_{1}}=\overline{U_{k}} .
$$

By collecting the results above, we get:

Proposition. The integrals $U_{k}$ of $\Omega_{1}$ over the b-cycles satisfy

$$
\operatorname{Im}\left(q \overline{U_{k}}\right)=\pi m_{k}, \quad m_{k} \in \mathbb{Z}, \quad k=1, \ldots g .
$$

Proof. By (5.2.16) and (5.2.19), we have

$$
\int_{b_{k}} \omega=-\bar{q} U_{k}+q \overline{U_{k}}=2 i \operatorname{Im}\left(q \overline{U_{k}}\right) .
$$

As in the proof of Lemma 5.2, we use the fact that the integral of $\omega$ over a closed cycle is an integer multiple of $2 \pi i$. This together with (5.2.21) proves (5.2.20).

5.3. Next we investigate the closing conditions in Theorem 3.9. Since $\beta^{2}$ is an even function in $\lambda$, we can write it as a function of $\nu=\lambda^{2}$. By Lemma 4.1, $a^{2}(\nu)$ has no zero of odd order on $S^{1}$. Therefore, by d') in Theorem 3.7, also $\beta^{2}=\frac{(\beta a)^{2}}{a^{2}}$ has no zeroes of odd order on $S^{1}$. As a function of $\lambda, \beta^{2}$ has a 
zero of order $2 n, n>0$, at $\lambda_{0} \in S^{1}$ iff as a function of $\nu$ it has a zero of order $2 n$ at $\nu_{0}=\lambda_{0}^{2}$. Since $\nu$ is a local coordinate around every point on $\mathcal{S}$, we have that $\beta^{2}$ has a zero of order $2 n$ at $\nu_{0}$ iff $\beta$ has a zero of order $n$ at each of the two covering points of $\nu_{0}$ on $\mathcal{C}$. We have proved the following:

Lemma. Let $\lambda_{0} \in S^{1}$ and let $P_{1}$ and $P_{2}$ be the two points on $\mathcal{C}$, such that $\nu\left(P_{1}\right)=\nu\left(P_{2}\right)=\lambda_{0}^{2}$. Then we have

a) $\beta^{2}(\lambda)$ vanishes at $\lambda_{0}$ iff $\beta$ vanishes at $P_{0}$ and $P_{1}$.

b) $\beta^{2}(\lambda)$ vanishes at least to fourth order at $\lambda_{0}$ iff $\beta$ vanishes at least to second order at $P_{0}$ and $P_{1}$.

Since $\beta\left(P_{0}\right)=-\beta\left(P_{1}\right)$, we have that in both statements of Lemma 5.3 we can replace "and" by "or". We can restate Theorem 3.9 in terms of $\omega$ :

Theorem. Let $\Psi: \mathbb{C} \rightarrow \mathbb{R}^{3}$ be a CMC-immersion in the r-dressing orbit of the cylinder with associated family $\left\{\Psi_{\lambda}, \lambda \in S^{1}\right\}$. Let $q \in \operatorname{Sym}(\Psi)=$ $\operatorname{Sym}\left(\Psi_{\lambda}\right), q \neq 0$, and let $\chi$ be defined as in Theorem 3.7. Let $\mathcal{C}$ be the hyperelliptic curve defined in Section 4.1 and let $\omega$ be the Abelian differential on $\mathcal{C}$ which is uniquely defined by Theorem 5.2. For $\lambda_{0} \in S^{1}$ denote by $P_{1}\left(\lambda_{0}\right)$ and $P_{2}\left(\lambda_{0}\right)$ the covering points of $\nu_{0}=\lambda_{0}^{2}$ on $\mathcal{C}$. Then the following are equivalent:

1. $q \in \operatorname{Per}\left(\Psi_{\lambda_{0}}\right)$.

2. $\beta$ vanishes at least to second order in $P_{1}\left(\lambda_{0}\right)$ and $P_{2}\left(\lambda_{0}\right)$.

3. The form $\omega$ has a zero at $P_{1}\left(\lambda_{0}\right)$ or $P_{2}\left(\lambda_{0}\right)$ and there exists a curve $\gamma$ on $\mathcal{C}^{*}$ connecting $P_{1}\left(\lambda_{0}\right)$ and $P_{2}\left(\lambda_{0}\right)$ such that

$$
\int_{\gamma} \omega=2 \pi i m, \quad m \in \mathbb{Z} .
$$

Proof. 1. $\Leftrightarrow 2$. follows from Theorem 3.9 and Lemma 5.3.

$2 . \Rightarrow 3$. Using (5.2.10) we get for each curve on $\mathcal{C}^{*}$ connecting $P_{1}$ and $P_{2}=I\left(P_{1}\right)$ :

$$
\int_{\gamma} \omega=\left.\ln (\alpha+\beta)\right|_{P_{1}} ^{P_{2}}=\ln \left((\alpha+\beta)\left(P_{2}\right)\right)-\ln \left((\alpha+\beta)\left(P_{1}\right)\right)
$$

where we have continued $\ln (\alpha+\beta)$ analytically along $\gamma$. Since $\beta\left(P_{1}\right)=$ $\beta\left(P_{2}\right)=0$,

$$
\int_{\gamma} \omega=\ln \left(\alpha\left(P_{2}\right)\right)-\ln \left(\alpha\left(P_{1}\right)\right)
$$

which by (4.6.3) is an integer multiple of $2 \pi i$. Thus, (5.3.1) holds. In addition, if $\beta$ vanishes to second order at $P_{1}$ and $P_{2}$, then also $\mathrm{d} \beta$ vanishes at both points. Therefore, $\omega=\alpha \mathrm{d} \beta-\beta \mathrm{d} \alpha$ has a zero at $P_{1}$ and $P_{2}$. 
3. $\Rightarrow 2$. Let $\gamma$ be a curve on $\mathcal{C}^{*}$ connecting $P_{1}$ and $P_{2}$ for which (5.3.1) holds. Then by (5.3.2) we get

$$
(\alpha-\beta)\left(P_{1}\right)=(\alpha+\beta)\left(P_{1}\right)
$$

from which with Proposition $4.6 \beta\left(P_{1}\right)=-\beta\left(P_{2}\right)=0$ follows. If in addition $\omega\left(P_{1}\right)=0$, then since $\beta\left(P_{1}\right)=0$, we get $\alpha\left(P_{1}\right)=1$ and $\mathrm{d} \beta\left(P_{1}\right)=0$. Thus $\beta$ vanishes to second order at $P_{1}$ and therefore also at $P_{2}$.

Remark. 1. It follows immediately from (3.1.1), that

$$
F(z,-\lambda)=F(-z, \lambda)
$$

i.e., $\Psi_{\lambda}$ and $\Psi_{-\lambda}$ differ only by a coordinate transformation. This explains, why the conditions 2 . and 3 . in Theorem 5.3 are invariant under the substitution $\lambda_{0} \rightarrow-\lambda_{0}$.

2. The proof of Theorem 5.3 shows, that in the third statement of the theorem, "there exists a curve $\gamma$ on $\mathcal{C}^{*}$ " can be replaced by "for all curves $\gamma$ on $\mathcal{C}^{* \prime}$.

5.4. If $\Psi$ is a periodic CMC-immersion with associated family $\left\{\Psi_{\lambda} ; \lambda \in\right.$ $\left.S^{1}\right\}$. then by Remark 2.7, $q \in \operatorname{Sym}\left(\Psi_{\lambda}\right)$ for all $\lambda \in S^{1}$. In Sections 4.1, 5.1 and 5.2 we have introduced a nonsingular hyperelliptic curve $\mathcal{C}$, a canonical homotopy basis $a_{1}, \ldots, a_{g}, b_{1}, \ldots, b_{g} \in H_{1}(\mathcal{C}, \mathbb{Z})$ and an Abelian differential $\Omega_{1}$ of the second kind on $\mathcal{C}$. If $q \in \operatorname{Sym}(\Psi)$, then these data satisfy Proposition 5.2. If for some $\lambda_{0} \in S^{1}, q \in \operatorname{Per}\left(\Psi_{\lambda_{0}}\right)$, then in addition the statement of Theorem 5.3 holds. In the special case that $\Psi_{\lambda_{0}}$ is a CMC-torus, we get

Theorem. Let $\Psi: \mathbb{C} \rightarrow \mathbb{R}^{3}$ be a periodic CMC-immersion with associated family $\left\{\Psi_{\lambda} ; \lambda \in S^{1}\right\}$. We define the nonsingular hyperelliptic curve $\mathcal{C}$ as in Section 4.1. Assume, that $\Psi_{\lambda_{0}}$ is a CMC-torus. Let $q_{j}=\frac{1}{2} Y_{j}+i \frac{1}{2} X_{j}$, $j=1,2$, be the generators of $\operatorname{Per}\left(\Psi_{\lambda_{0}}\right)$. We introduce a homotopy basis $a_{1}, \ldots, a_{g}, b_{1}, \ldots b_{g}$, the Abelian differential $\Omega_{1}$ and $U_{k}=\alpha_{k}+i \beta_{k}$ as in Sections 5.1 and 5.2. Then there exists a curve $\gamma$ on $\mathcal{C}^{*}$ connecting the points $P_{1}\left(\lambda_{0}\right)$ and $P_{2}\left(\lambda_{0}\right)$ for which $\nu\left(P_{i}\left(\lambda_{0}\right)\right)=\lambda_{0}^{2}$, such that for $X_{1}, Y_{1}, X_{2}, Y_{2}$, $\alpha_{k}, \beta_{k}, k=1, \ldots, g$, defined above and $c_{1}, c_{2}$ defined by

$$
c_{1}+i c_{2}=\frac{1}{2} \int_{\gamma} \Omega_{1} .
$$

1. The matrix

$$
\frac{1}{2 \pi}\left(\begin{array}{cc}
X_{1} & Y_{1} \\
X_{2} & Y_{2}
\end{array}\right)\left(\begin{array}{cc}
2 c_{1} & \alpha_{1}, \ldots, \alpha_{g} \\
-2 c_{2} & -\beta_{1}, \ldots,-\beta_{g}
\end{array}\right)
$$

has integer entries and 
2. $\Omega_{1}$ vanishes at $P_{1}\left(\lambda_{0}\right)$ (and therefore also at $P_{2}\left(\lambda_{0}\right)$ ).

Proof. Let $\Psi_{\lambda_{0}}$ be a CMC-torus, such that $q_{1}$ and $q_{2}$ generate $\operatorname{Per}\left(\Psi_{\lambda_{0}}\right)$. If we define $\alpha_{k}, \beta_{k}, k=1, \ldots, g$, as above then by Proposition 5.2 we have for $j=1,2$ :

$$
\pi m_{k}=\operatorname{Im}\left(q_{j} \overline{U_{k}}\right)=\frac{1}{2}\left(X_{j} \alpha_{k}-Y_{j} \beta_{k}\right),
$$

where $m_{k} \in \mathbb{Z}$. This shows, that the last $g$ columns in the matrix product of (5.4.2) have integer entries.

In addition, if we set $V=\int_{\gamma} \Omega_{1}$, then by Theorem 5.3, we get for $j=1,2$ :

$$
2 i \operatorname{Im}\left(q_{j} \bar{V}\right)-\overline{q_{j}} V+q_{j} \bar{V}=2 i\left(X_{j} c_{1}-Y_{j} c_{2}\right)=2 \pi i m_{j}, \quad m_{j} \in \mathbb{Z} .
$$

This shows, that the first column of (5.4.2) has integer entries, proving the first part of the statement.

Finally, by Theorem 5.3 and (5.2.16), we get that $-\overline{q_{1}} \Omega_{1}+q_{1} \overline{\Omega_{1}}$ and $-\overline{q_{2}} \Omega_{1}+q_{2} \overline{\Omega_{1}}$ vanish at $P_{1}\left(\lambda_{0}\right)$ or $P_{2}\left(\lambda_{0}\right)$, and therefore, by (5.2.17), at both points. Since $q_{1}$ and $q_{2}$ are linearly independent as vectors in $\mathbb{R}^{2}$, it follows that $\Omega_{1}$ vanishes at $P_{1}\left(\lambda_{0}\right)$.

Remark. This reproduces the conditions (6.13) and (6.14) of [1] for CMCtori.

5.5. In the last two sections we have reformulated the necessary conditions of Theorem 3.7 for periodic surfaces in terms of algebro-geometric data on $\mathcal{C}$. For CMC-tori, the result is the same as in [1, Theorem 6.1], as expected. It remains to construct periodic surfaces from algebro-geometric data.

We start with the following data:

1. Let $g \in \mathbb{N}$ and let $\nu_{2 k-1} \in \mathbb{C}, k=1, \ldots, g$, be mutually distinct, such that $0<\left|\nu_{2 k-1}\right|<1$,

2. let $q \in \mathbb{C}^{*}$.

Define the nonsingular hyperelliptic curve $\mathcal{C}$ by

$$
\mu^{2}=\nu \prod_{k=1}^{2 g}\left(\nu-\nu_{k}\right), \quad \nu_{2 k}={\overline{\nu_{2 k-1}}}^{-1}, \quad k=1, \ldots, g .
$$

Then $g$ is the genus of $\mathcal{C}$ and $\mathcal{C}$ has no branchpoints over the unit circle. For this surface, Theorem 4.1 holds. The surface $\mathcal{C}$ admits the involution $\hat{\sigma}$ defined in (4.3.2). As in Section 4.3, we define for a scalar function $f$ on $\mathcal{C}, f^{*}=\overline{f \circ \hat{\sigma}}$. We denote by $P_{0}$ and $P_{\infty}$ the branchpoints of $\mathcal{C}$ over $\nu=0$ 
and $\nu=\infty$. We also define a standard homotopy basis $a_{1}, \ldots, a_{g}, b_{1}, \ldots, b_{g}$, such that (5.1.4) and (5.1.5) hold. Let $\Omega_{1}$ be the uniquely defined Abelian differential on $\mathcal{C}$ which is holomorphic except at $P_{\infty}$ and satisfies (5.2.13) and (5.2.12). We also define $\Omega_{2}$ by (5.2.14). We denote the integral of $\Omega_{1}$ over the cycle $b_{k}$ by $U_{k}$.

We now assume, that with these definitions the equation

$$
\operatorname{Im}\left(q \overline{U_{k}}\right)=\pi m_{k}, \quad m_{k} \in \mathbb{Z}, \quad k=1, \ldots g,
$$

is satisfied. From these data we will construct a family of CMC-immersions which are periodic w.r.t. translation by $q$.

For later use we also introduce the hyperelliptic curve $\mathcal{C}^{\prime}$ defined by

$$
\tilde{\mu}^{2}=\prod_{k=1}^{2 g}\left(\lambda-\sqrt{\nu_{k}}\right)\left(\lambda+\sqrt{\nu_{k}}\right) .
$$

The discussion in Sections 4.2-4.5 applies to $\mathcal{C}$ and $\mathcal{C}^{\prime}$. In particular, we have the local coordinate $\lambda=\lambda(\nu, \mu)$ at $P_{0}$ on $\mathcal{C}$ (see also the proof of Lemma 5.2).

5.6. Next we define the Abelian differential $\omega$ by (5.2.16). By the definition of $\Omega_{1}$ and $\Omega_{2}$, it satisfies (5.2.7) and (5.2.6). It has poles at $P_{0}$ and $P_{\infty}$. Its behaviour at the singularities is given by (5.2.4), (5.2.5). Let $I$ be the hyperelliptic involution on $\mathcal{C}$. Since $-I^{*} \omega$ also satisfies (5.2.4)-(5.2.6), we have

$$
I^{*} \omega=-\omega
$$

We define the multi-valued holomorphic function $p$ on $\mathcal{C}^{*}=\mathcal{C} \backslash\left\{P_{0}, P_{\infty}\right\}$ by

$$
p(\nu, \mu)=\int_{\gamma(\nu, \mu)} \omega
$$

where $\gamma(\nu, \mu)$ is a curve connecting the branchpoint over $\nu_{1}$ with $(\nu, \mu)$. By (5.2.6), (5.5.2) and (5.2.21), $p$ is at every point of $\mathcal{C}^{*}$ defined up to an integer multiple of $2 \pi i$. Moreover, every branch of $p$ is locally meromorphic at each point of $\mathcal{C}^{*}$. The functions

$$
\alpha=\cosh (p), \quad \beta=\sinh (p)
$$

are holomorphic and single-valued on $\mathcal{C}^{*}$. Clearly, $\alpha^{2}-\beta^{2}=1$ on $\mathcal{C}^{*}$. By (5.6.1), we have modulo $2 \pi i \mathbb{Z}$ :

$$
(p \circ I)(\nu, \mu)=\int_{\gamma(\nu,-\mu)} \omega=\int_{\gamma(\nu, \mu)}\left(I^{*} \omega\right)=-p(\nu, \mu),
$$


where we have chosen $\gamma(\nu, \mu)=I \circ \gamma(\nu,-\mu)$. By (5.2.7), we have modulo $2 \pi i \mathbb{Z}$ :

$$
(p \circ \hat{\sigma})(\nu, \mu)=\int_{\gamma(\hat{\sigma}(\nu, \mu))} \omega=\int_{\gamma(\nu, \mu)}\left(\hat{\sigma}^{*} \omega\right)=-\int_{\gamma(\nu, \mu)} \bar{\omega}=-\overline{p(\nu, \mu)},
$$

where we have chosen $\gamma(\nu, \mu)=\hat{\sigma} \circ \gamma(\hat{\sigma}(\nu, \mu))$. This shows, that $p^{*}=-p$ modulo $2 \pi i \mathbb{Z}$. Therefore, we get

$$
\alpha^{*}=\alpha, \quad \beta^{*}=-\beta
$$

and

$$
\alpha \circ I=\alpha, \quad \beta \circ I=-\beta .
$$

Thus, $\alpha$ and $\beta^{2}$ project down to holomorphic functions $\alpha(\nu), \beta^{2}(\nu)$ on $\mathbb{C}_{\nu}^{*}$ which are real on $S^{1}$. Pulling back $\omega, p, \alpha$ and $\beta$ from $\mathcal{C}^{*}$ to $\mathcal{C}^{\prime *}$ and from $\mathbb{C}_{\nu}^{*}$ to $\mathbb{C}_{\lambda}^{*}$, respectively, yields holomorphic functions $\alpha(\lambda)$ and $\beta^{2}(\lambda)$, which are real on $S^{1}$ in view of (5.6.6). Moreover, (5.6.7) and (5.6.6) show, that $\alpha$ and $\beta^{2}$ are even on $\mathbb{C}_{\lambda}^{*}$ and that $\beta$ is non-positive over $S^{1}$ by Lemma 4.3.

The function $p$ on $\mathcal{C}^{\prime *}$ can be written locally near one of the points on $\mathcal{C}^{\prime}$ above $\lambda=0$ in the form $p(\lambda)=\lambda^{-1} q-\lambda \bar{q}+f_{+}$, where $f_{+}$is locally holomorphic at $\lambda=0$ and, by (5.6.5), an odd function of $\lambda$.

We collect these results in the following:

Lemma. With the notation of Section 5.5 we have: Let $0<r_{0}<1$ be such that $\mathcal{C}^{\prime}$ has no branchpoints over $I^{\left(r_{0}\right)}$. Define

$$
p(\nu, \mu)=\int_{\gamma(\nu, \mu)}\left(-\bar{q} \Omega_{1}+q \overline{\hat{\sigma}^{*} \Omega_{1}}\right)
$$

where $\gamma(\nu, \mu)$ is an arbitrary curve joining $\left(\nu_{1}, 0\right)$ and $(\nu, \mu)$ on $\mathcal{C}$. Then $p$ is a multivalued function on $\mathcal{C}$ unbranched over $\nu \in I^{\left(r_{0}\right)}$. Every branch of $p$ can be identified with an odd meromorphic function on $\lambda \in I^{\left(r_{0}\right)}$, which is of the form

$$
p(\lambda)=\lambda^{-1} q-\lambda \bar{q}+f_{+}(\lambda)
$$

where $f_{+}(\lambda)$ is odd in $\lambda$ and holomorphic on $I^{\left(r_{0}\right)}$, i.e., $f_{+} \in \mathcal{A}_{r_{0}}^{+}$. If we define $\alpha(\lambda)=\cosh (p(\lambda)), \beta(\lambda)=\sinh (p(\lambda))$, then $\alpha$ and $\beta$ satisfy the conditions $\left.\mathrm{a}^{\prime}\right)-\mathrm{c}^{\prime}$ ) of Theorem 3.8. Furthermore, $\beta$ vanishes at all branchpoints of $\mathcal{C}$,

$$
\beta\left(\nu_{k}, 0\right)=0, \quad k=1, \ldots, 2 g .
$$


Proof. It only remains to prove (5.6.10). But this follows immediately from (5.6.7) since the branchpoints are fixed points under the hyperelliptic involution $I$ on $\mathcal{C}$.

5.7. It remains to construct rational functions $a^{2}(\lambda), b^{2}(\lambda), c^{2}(\lambda)$, such that the conditions a)-e) and d') of Theorem 3.8 are met. We will first construct $a^{2}(\lambda)$.

If the genus $g$ of $\mathcal{C}$ is odd, we define

$$
\hat{a}^{2}(\nu)=\left(\prod_{k=1}^{g} \nu_{2 k-1}\right) \nu^{g} \prod_{k=1}^{2 g}\left(\nu-\nu_{k}\right)^{-1} .
$$

If $g$ is even, we choose an arbitrary $\nu_{0} \in S^{1}$ and define

$$
\hat{a}^{2}(\nu)=\nu_{0}\left(\prod_{k=1}^{g} \nu_{2 k-1}\right) \nu^{g-1}\left(\nu-\nu_{0}\right)^{2} \prod_{k=1}^{2 g}\left(\nu-\nu_{k}\right)^{-1} .
$$

We note, that in both cases $\hat{a}^{2}$ is a rational function with a zero of odd order at $\nu=0$ and simple poles precisely at the points $\nu_{k}, k=1, \ldots, 2 g$. A simple calculation gives

$$
\left(\hat{a}^{2}\right)^{*}(\nu)=\overline{\hat{a}^{2}\left(\bar{\nu}^{-1}\right)}=\hat{a}^{2}(\nu) .
$$

In addition, $\hat{a}^{2}$ has no zeroes of odd order different from $\nu=0$. In particular, since $\hat{a}^{2}$ is real on $S^{1}$ with at most one zero on $S^{1}$, it is either non-negative or non-positive on $S^{1}$. Therefore, there exists a real constant $0<A<\infty$, such that $0 \leq \hat{a}^{2}(\nu) \leq A$ or $-A \leq \hat{a}^{2}(\nu) \leq 0$ for all $\nu \in S^{1}$. Let us define the rational function

$$
a_{0}^{2}=\epsilon \hat{a}^{2}
$$

where $\epsilon= \pm 1$ is chosen such that $a_{0}^{2}$ is non-negative on $S^{1}$.

Proposition. Let $\mathcal{C}, \mathcal{C}^{\prime}, \beta$ and $0<r_{0}<1$ be defined as in Lemma 5.6. Then $a_{0}^{2}(\nu)$ constructed above is a rational even function of $\lambda$, which is real and non-negative on $S^{1}$. Furthermore, $a_{0}^{2}(\lambda)$ restricts to the square of an odd holomorphic function $a_{0}(\lambda) \in \mathcal{A}_{r_{0}}^{+}$, such that $\left(\beta a_{0}\right)(\lambda)$ can be extended holomorphically to an even function on $\mathbb{C}_{\lambda}^{*}$.

Proof. We have already shown, that $a_{0}^{2}$ is rational in $\nu=\lambda^{2}$ and therefore an even rational function of $\lambda$. From (5.7.3) and (5.7.4), it follows, that $a_{0}^{2}$ is real and non-negative on $S^{1}$. Since $a_{0}^{2}$ has only a zero of odd order at $\nu=0$ and only poles of odd order at $\nu_{k}, k=1, \ldots, 2 g$, its square root $a_{0}$ is a meromorphic function on $\mathcal{C}$, which satisfies $a_{0} \circ I=-a_{0}$. It has simple 
poles precisely at the branchpoints of $\mathcal{C}^{*}$ and has zeroes of odd order at $P_{0}$ and $P_{\infty}$. Applying the usual identifications between functions on $I^{\left(r_{0}\right)}$, functions on a patch of $\mathcal{C}^{\prime}$ above $I^{\left(r_{0}\right)}$ and functions in a neighbourhood of $P_{0}$ on $\mathcal{C}$, and also using Lemma 4.4 we conclude that $a_{0}$ is an odd holomorphic function of $\lambda$ on $I^{\left(r_{0}\right)}$, i.e., $a_{0} \in \mathcal{A}_{r_{0}}^{+}$. In addition, since $\beta$ is holomorphic on $\mathcal{C}^{*}$ and, by (5.6.10), vanishes at the branchpoints of $\mathcal{C}^{*}$, the function $\beta a_{0}$ is holomorphic on $\mathcal{C}^{*}$ and, by (5.6.7) invariant under $I$. Therefore, $\beta a_{0}$ projects down to an even holomorphic function $\left(\beta a_{0}\right)(\lambda)$ on $\mathbb{C}^{*}$.

In the following, for $x \in \mathbb{R}$ we denote by $[x]$ the greatest integer less than or equal to $x$.

Lemma. Let $\mathcal{C}, \mathcal{C}^{\prime}, \beta$ and $0<r_{0}<1$ be defined as in Theorem 5.6. Let $\tilde{f}(\nu)$ be a rational function which is holomorphic on $\mathbb{C}^{*}$, real on $S^{1}$, and has a pole of order at most $\left[\frac{g-1}{2}\right]$ at $\nu=0$. Let us define $a_{0}^{2}$ as above and set $a^{2}=\tilde{f}^{2} a_{0}^{2}$. For each such $\tilde{f}, a^{2}$ is an even rational function of $\lambda$ which is real and non-negative on $S^{1}$. Furthermore, the restriction of $a^{2}$ to $C_{r_{0}}$, the circle with radius $r_{0}$ with center $\lambda=0$, is the square of an odd function $a \in \mathcal{A}_{r_{0}}^{+}$. In addition, $\beta a$ extends to an even holomorphic function $(\beta a)(\lambda)$ on $\mathbb{C}^{*}$.

Proof. By Proposition 5.7, the functions $\tilde{f}^{2}, a_{0}^{2}$ and therefore also $a^{2}$ are even rational functions of $\lambda$, which are real and non-negative on $S^{1}$. In addition, the restriction of $a_{0}^{2}$ to $C_{r_{0}}$ is the square of an odd function $a_{0}(\lambda) \in \mathcal{A}_{r_{0}}^{+}$. Thus, since $\tilde{f}$ is even in $\lambda$, and since $\tilde{f}^{2} a^{2}$ has no poles on $I^{\left(r_{0}\right)}$, the restriction of $a^{2}$ to $C_{r_{0}}$ is the square of the odd function $a(\lambda)=\tilde{f}(\lambda) a_{0}(\lambda) \in \mathcal{A}_{r_{0}}^{+}$. Furthermore, $\tilde{f}, \beta a_{0}$ and therefore also $\beta a=\tilde{f} \cdot\left(\beta a_{0}\right)$ are even holomorphic functions of $\lambda$ on $\mathbb{C}^{*}$.

Theorem. Let $\mathcal{C}, \mathcal{C}^{\prime}, \beta$ and $0<r_{0}<1$ be defined as in Theorem 5.6. Then there exists at least a real g-parameter family of even rational functions $a^{2}(\lambda)$, such that

a) $a^{2}$ is real on $S^{1}$ and $0 \leq a^{2}(\nu)<1$ for $\nu \in S^{1}$,

b) the restriction of $a^{2}$ to $C_{r_{0}}$ is the square of an odd function $a(\lambda) \in \mathcal{A}_{r_{0}}^{+}$,

c) $(\beta a)(\lambda)$ can be extended to an even holomorphic function on $\mathbb{C}^{*}$.

Proof. By Lemma 5.7, to each rational function $\tilde{f}(\nu)$ which is real on $S^{1}$, holomorphic on $\mathbb{C}^{*}$, and has a pole of order at most $\left[\frac{g-1}{2}\right]$ we can construct an even rational function $a^{2}(\lambda)$ which satisfies b) and c), is real and nonnegative on $S^{1}$ and has no poles on $S^{1}$. Thus, we can always normalize $a^{2}$ such that $a^{2}(\nu)<1$ for all $\nu \in S^{1}$. Then also a) is satisfied.

Since $\tilde{f}$ is real on $S^{1}$, we have, by Section $2.8, \tilde{f}^{*}=\tilde{f}$. Therefore, $\tilde{f}$ has the same pole order at $\nu=0$ and $\nu=\infty$. As a consequence, with $m=\left[\frac{g-1}{2}\right]$, 
$\tilde{f}$ is of the form $\lambda^{-m}\left(r_{0}+r_{1} \lambda+\ldots+r_{m-1} \lambda^{m-1}\right)+t_{0}+t_{1} \lambda+\ldots+t_{m} \lambda^{m}$. This implies that $\tilde{f}$ is defined by $2\left[\frac{g-1}{2}\right]+1$ complex parameters. After imposing the reality condition there are still $2\left[\frac{g-1}{2}\right]+1$ real parameters left free, which determine the chosen admissible function $\tilde{f}$ completely. If $g$ is odd, then $2\left[\frac{g-1}{2}\right]=g-1$. If $g$ is even, then $2\left[\frac{g-1}{2}\right]=g-2$. In the case that $g$ is even we have the additional freedom to choose a zero $\nu_{0} \in S^{1}$ of $a_{0}^{2}$. Altogether, in both cases this gives a real $g$-parameter family of functions $a^{2}$, which satisfy a) - c).

5.8. It remains to construct the even rational functions $b^{2}$ and $c^{2}$, such that all conditions of Theorem 3.8 are satisfied.

Lemma. Let $a^{2}(\lambda)$ and $0<r_{0}<1$ be as in Theorem 5.7. Then the equation

$$
b^{2}\left(b^{2}\right)^{*}=\left(1-a^{2}\right)^{2},
$$

has a solution $b^{2}(\lambda)$ which is rational, even in $\lambda$, and its restriction to $C_{r_{0}}$ is the square of an even function $b(\lambda) \in \mathcal{A}_{r_{0}}^{+}$. Furthermore, we can choose $b^{2}$ such that $b^{2}$ and $\left(b^{2}\right)^{*}$ both have either a simple zero or a simple pole at each of the branchpoints $\nu_{1}, \ldots, \nu_{2 g}$ of $\mathcal{C}$ and no other poles or zeroes of odd order.

Proof. With $a^{2}$ also $1-a^{2}$ is rational, even in $\lambda$, and defined and real on $S^{1}$. Furthermore, $1-a^{2}$ has no zeroes on $S^{1}$. By reordering the branchpoints $\nu_{1}, \ldots, \nu_{2 g}$ if necessary we can assume that $a^{2}$ has simple poles at $\nu_{1}, \ldots, \nu_{2 K}$ and zeroes of odd order at $\nu_{2 K+1}, \ldots, \nu_{2 g}$, where $g-\left[\frac{g-1}{2}\right] \leq K \leq g$. Here, the lower bound for the number of poles of $a^{2}$ follows from the maximum number $2\left[\frac{g-1}{2}\right]$ of zeroes of the rational function $\tilde{f}$ in Lemma 5.7. We write

$$
1-a^{2}=\gamma \frac{\prod_{j=1}^{n_{1}}\left(\nu-\nu_{j}^{(1)}\right)\left(\nu-\overline{\nu_{j}^{(1)}}-1\right)}{\prod_{k=1}^{K}\left(\nu-\nu_{2 k-1}\right)\left(\nu-\nu_{2 k}\right)}, \quad \gamma \in \mathbb{R},
$$

where $\left\{\nu_{j}^{(1)},{\overline{\nu_{j}^{(1)}}}^{-1}\right\}$ are the zeroes of $1-a^{2}$. Since $\left(1-a^{2}\right)(\lambda=0)=$ $\left(1-a^{2}\right)^{*}(\lambda=\infty)=1$, we have $n_{1}=K$ and $\nu_{j}^{(1)} \neq 0$ for all $j=1, \ldots, n_{1}$. Define

$$
\tilde{b}^{2}=\prod_{k=1}^{K} \frac{\left(\nu-\nu_{k}^{(1)}\right)^{2}}{\left(\nu-\nu_{2 k-1}\right)\left(\nu-\nu_{2 k}\right)} \prod_{j=K+1}^{g} \frac{\nu-\nu_{2 j-1}}{\nu-\nu_{2 j}}
$$

then a straightforward computation shows that there exists a positive real constant $\delta$, such that $\left(1-a^{2}\right)^{2}=\delta\left(\tilde{b}^{2}\right)\left(\tilde{b}^{2}\right)^{*}$. If we define $b^{2}=\sqrt{\delta} \tilde{b}^{2}$ then $b^{2}$ 
is rational and satisfies (5.8.1). Also, $b^{2}$ has a simple pole or a simple zero at each of the points $\nu_{1}, \ldots, \nu_{2 g}$ and no other poles or zeroes of odd order. The function $\left(b^{2}\right)^{*}$ is up to a complex constant given by

$$
\prod_{k=1}^{K} \frac{\left(\nu-\bar{\nu}_{k}^{(1)}-1\right.}{\left(\nu-\nu_{2 k-1}\right)\left(\nu-\nu_{2 k}\right)} \prod_{j=K+1}^{g} \frac{\nu-\nu_{2 j}}{\nu-\nu_{2 j-1}},
$$

and has therefore also simple poles or simple zeroes at $\nu_{1}, \ldots, \nu_{2 g}$ and no other poles or zeroes of odd order.

Since $b^{2}$ has no poles and no zeroes of odd order in $I^{\left(r_{0}\right)}$, its restriction to $C_{r_{0}}$ is the square of a function $b(\lambda) \in \mathcal{A}_{r_{0}}^{+}$. Since we have written $b^{2}$ in terms of $\nu$, it is clear that $b^{2}$ is an even function on $\mathbb{C}_{\lambda}$. Thus, $b(\lambda)$ is even or odd. Since $b^{2}$ does not vanish at $\lambda=0$, we get that $b(\lambda=0) \neq 0$ whence $b(\lambda)$ is even.

Proposition. Let $\mathcal{C}$ and $q$ be defined as in Section 5.5. Define functions $f_{+} \in \mathcal{A}_{r_{0}}^{+}, 0<r_{0}<1, p=\lambda^{-1} q-\lambda \bar{q}+f_{+}, \alpha=\cosh (p), \beta=\sinh (p)$ as in Lemma 5.6. Furthermore, let $a^{2}(\lambda)$ be defined as in Theorem 5.7. Then there exist two even rational functions $b^{2}(\lambda), c^{2}(\lambda)$, such that all the conditions of Theorem 3.8 are satisfied for some $r^{\prime}=r=r_{0}$.

Proof. By Lemma 5.6, there exists $0<r_{0}<1$, such that the functions $f_{+}$, $p, \alpha$ and $\beta$ satisfy Conditions a') $-\mathrm{c}^{\prime}$ ) of Theorem 3.8 for $r^{\prime}=r_{0}$, where the maximal radius $r_{0}$ is determined by the surface $\mathcal{C}$. By a),b) in Theorem 5.7, each function $a^{2}$ constructed in Theorem 5.7 satisfies Conditions a) and c) of Theorem 3.8 for $r=r_{0}$. Furthermore, by c) in Theorem 5.7, $(\beta a)(\lambda)$ can be extended holomorphically to $\mathbb{C}^{*}$. We define the even rational function $b^{2}(\lambda)$ as in (5.8.3) and use Condition b) of Theorem 3.8 to define $c^{2}$. Clearly, $c^{2}(\lambda)$ is an even rational function which has the same pole divisor as $b^{2}$ and $a^{2}$ and only zeroes of even order. Since $c^{2}$ has no pole and no zero of odd order on $I^{\left(r_{0}\right)}$, its restriction to $C_{r_{0}}$ is a function $c(\lambda) \in \mathcal{A}_{r_{0}}^{+}$. This function is even or odd in $\lambda$. Since $b^{2}$ does not vanish at $\lambda=\infty, c^{2}(\lambda=0) \neq 0$ and $c$ is an even function in $\lambda$. This shows, that Condition c) and d) of Theorem 3.8 are satisfied. By (5.8.1), $b^{2} c^{2}=\left(1-a^{2}\right)^{2}$. We choose the sign of the square roots $b$ and $c$ of $b^{2}$ and $c^{2}$, such that $b c=1-a^{2}$. Then also Condition e) of Theorem 3.8 is satisfied.

By Lemma $5.8, b^{2}$ and $c^{2}$ have either a simple pole or a simple zero at each branchpoint $\nu_{1}, \ldots, \nu_{2 g}$. Since $\beta^{2}$ is holomorphic on $\mathbb{C}_{\nu}^{*}$ and vanishes at each of the points $\nu_{1}, \ldots, \nu_{2 g}, \beta^{2} b^{2}$ and $\beta^{2} c^{2}$ are holomorphic functions on $\mathbb{C}_{\nu}^{*}$. Furthermore, since by Theorem $5.7, \beta^{2} a^{2}$ is the square of a holomorphic function on $\mathbb{C}_{\nu}^{*}$, the zero of $\beta^{2}$ at each branchpoint is of odd order and $\beta^{2}$ 
can have no further zeroes of odd order on $\mathbb{C}_{\nu}^{*}$. Thus, $\beta^{2} b^{2}$ and $\beta^{2} c^{2}$ have no zeroes of odd order, whence are squares of holomorphic functions $\beta b$ and $\beta c$ on $\mathbb{C}_{\nu}^{*}$.

Theorem. Let $\mathcal{C}$ be defined as in Section 5.5. Choose a homotopy basis $a_{1}, \ldots, a_{g}, b_{1}, \ldots, b_{g}$ and the Abelian differential $\Omega_{1}$ as in Section 5.5, such that (5.5.2) holds for some $q \in \mathbb{C}^{*}$. Then there exists $0<r_{0}<1$ and a real g-parameter family $\mathcal{S}_{\mathcal{C}}$ of CMC-immersions, such that each such immersion $\Psi \in \mathcal{S}_{\mathcal{C}}$ lies in the $r_{0}$-dressing orbit of the cylinder, $q \in \operatorname{Sym}(\Psi)$, and the hyperelliptic curve associated to $\Psi$ in Section 4.1 is $\mathcal{C}$. The family $\mathcal{S}_{\mathcal{C}}$ depends only on $\mathcal{C}$, not on the chosen solution of (5.5.2).

Proof. By Theorem 5.7 and Proposition 5.8, to each hyperelliptic curve $\mathcal{C}$ and constant $q$, satisfying the conditions of Section 5.5 , there exists $0<r_{0}<1$, functions $f_{+} \in \mathcal{A}_{r_{0}}^{+}, p=\lambda^{-1} q-\lambda \bar{q}+f_{+}, \alpha=\cosh (p), \beta=\sinh (p), a^{2}, b^{2}$, $c^{2}$, such that all assumptions of Theorem 3.8 are satisfied for $r^{\prime}=r=r_{0}$. From Theorem 5.7 we know that there exists at least a $g$-parameter family of such data. Therefore, by the statement of Theorem 3.8, there exists a real $g$ parameter family $\mathcal{S}_{\mathcal{C}}$ of CMC-immersions, such that each $\Psi \in \mathcal{S}_{\mathcal{C}}$ is generated by dressing the cylinder with some $h_{+} \in \Lambda_{r^{\prime \prime}}^{+} \mathbf{S L}(2, \mathbb{C})_{\sigma}$ with $0<r^{\prime \prime} \leq r_{0}$ and $h_{+} A h_{+}^{-1}=\left(\begin{array}{cc}a & b \\ c & -a\end{array}\right)$. Since all functions $a^{2}$ constructed in Theorem 5.7 are squares of a meromorphic function $a$ on the same hyperelliptic curve $\mathcal{C}$, they all yield the surface $\mathcal{C}$ under the construction in Section 4.1.

The construction of $a^{2}, b^{2}$ and $c^{2}$ in Sections 5.7 and 5.8, depends only on $\mathcal{C}$ not on the constant $q$. I.e., if there are two constants $q_{1}, q_{2} \in \mathbb{C} *$ both satisfying (5.5.2), then for both constants we get the same $0<r_{0}<1$ and $g$-parameter family of matrices $h_{+} \in \Lambda_{r^{\prime \prime}}^{+} \mathbf{S L}(2, \mathbb{C})_{\sigma}, r^{\prime \prime} \leq r_{0}$. Therefore, the family $\mathcal{S}_{\mathcal{C}}$ doesn't depend on the chosen solution $q$ of (5.5.2).

Corollary. Let $\mathcal{C}, \Omega_{1}, q \in \mathbb{C}^{*}$ and $\Psi \in \mathcal{S}_{\mathcal{C}}$ be given as in Theorem 5.8 and define $\omega$ by (5.2.16). For $\lambda_{0} \in S^{1}$ denote by $P_{1}\left(\lambda_{0}\right)$ and $P_{2}\left(\lambda_{0}\right)$ the covering points of $\nu_{0}=\lambda_{0}^{2}$ on $\mathcal{C}$. Then the following are equivalent:

1. $q \in \operatorname{Per}\left(\Psi_{\lambda_{0}}\right)$,

2. $\omega$ has a zero at $P_{1}\left(\lambda_{0}\right)$ or $P_{2}\left(\lambda_{0}\right)$ and there exists a curve $\gamma$ on $\mathcal{C}^{*}$ connecting $P_{1}\left(\lambda_{0}\right)$ and $P_{2}\left(\lambda_{0}\right)$, such that $\int_{\gamma} \omega=2 \pi i m, \quad m \in \mathbb{Z}$.

Proof. By Theorem 5.8, $q \in \operatorname{Sym}(\Psi)=\operatorname{Sym}\left(\Psi_{\lambda}\right)$ for all $\lambda \in S^{1}$, and the curve defined for $\Psi$ in Section 4.1 coincides with $\mathcal{C}$. Thus, the equivalence follows immediately from Theorem 5.3.

5.9. Theorem 5.8 together with Corollary 5.8 allows us to classify periodic surfaces in terms of algebro-geometric data: If $q \in \operatorname{Per}\left(\Psi_{\lambda_{0}}\right), q \neq 0$, then 
$\operatorname{Per}\left(\Psi_{\lambda_{0}}\right) \neq\{0\}$, and, by Theorem 1.2 , there exists a Riemann surface $M$ with universal cover $\pi: \mathbb{C} \rightarrow M$ and elementary group $\Gamma=\operatorname{Per}\left(\Psi_{\lambda_{0}}\right)$ such that $\Psi_{\lambda_{0}}=\Phi \circ \pi$ defines a CMC-immersion $\Phi: M \rightarrow \mathbb{R}^{3}$. In particular, if $\operatorname{Per}\left(\Psi_{\lambda_{0}}\right)$ is generated by only one translation, then $M$ is topologically a cylinder. If there exist two linearly independent translations in $\operatorname{Per}\left(\Psi_{\lambda_{0}}\right)$, then $M$ is topologically a torus. By part 3 . in Theorem 1.2, these are the only possible cases. As a special case we get:

Theorem. Let $\mathcal{C}, \Omega_{1}$ and $U_{k}$ be defined as in Section 5.5. If there exists a real constant $\phi \in[0,2 \pi)$, such that

$$
\operatorname{Re}\left(U_{k}\right) \sin (\phi)-\operatorname{Im}\left(U_{k}\right) \cos (\phi)=0, \quad k=1, \ldots, g,
$$

then the associated family of each $\Psi$ constructed in Theorem 5.8 contains a Delaunay surface.

Conversely, if $\Psi(\mathbb{C})$ is in the associated family of a Delaunay surface, and if $\mathcal{C}$ and $U_{k}$ are defined as in Sections 4.1 and 5.2, then there exists $\phi \in[0,2 \pi)$, such that (5.9.1) is satisfied.

Proof. If $U_{k}$ is real, then for all real $q \in \mathbb{C},(5.5 .2)$ is satisfied with $m_{k}=0$, $k=1, \ldots, g$. Thus, $\operatorname{Sym}(\Psi)$ contains the one-parameter subgroup $\mathbb{R} \subset \mathbb{C}$. By Theorem 1.2 this implies, that $\Psi$ is in the associated family of a Delaunay surface.

Conversely, if $\Psi(\mathbb{C})$ is a Delaunay surface, then, by [4, Lemma 2.15], $\operatorname{Sym}(\Psi)$ contains a one-parameter subgroup generated by some $q_{0} \in \mathbb{C}^{*}$, $\left|q_{0}\right|=1$. If we write $q_{0}=e^{i \phi}$ then (5.5.2) holds for all $q$ of the form $q=r e^{i \phi}$, $r \in \mathbb{R}$. Thus, $\operatorname{Im}\left(e^{i \phi} \overline{U_{k}}\right)=0$ for all $k=1, \ldots, g$, which gives (5.9.1).

5.10. Let us again consider the case of CMC-tori.

Theorem. Let $\mathcal{C}, \Omega_{1}, U_{k}, k=1, \ldots, g$, and $q_{1}=q \in \mathbb{C}^{*}$ be given as in Theorem 5.8. Assume that there exists a second constant $q_{2} \in \mathbb{C}^{*}$, linearly independent of $q_{1}$, such that also for $q_{2}$ Equation (5.5.2) is satisfied. Let $\lambda_{0} \in S^{1}$ and choose an arbitrary curve $\gamma$ in $\mathcal{C}^{*}$ connecting the two points $P_{1}\left(\lambda_{0}\right)$ and $P_{2}\left(\lambda_{0}\right)$, such that $\nu\left(P_{i}\left(\lambda_{0}\right)\right)=\lambda_{0}^{2}$. Define $X_{1}, Y_{1}, X_{2}, Y_{2}, \alpha_{k}, \beta_{k}$, $k=1, \ldots, g, c_{1}, c_{2}$ as in Theorem 5.4. If 1. and 2. of Theorem 5.4 are satisfied, then there exists $0<r_{0}<1$ and a real $g-1$-parameter family $\mathcal{T}_{\mathcal{C}}$ of CMC-tori in the $r_{0}$-dressing orbit of the cylinder, such that for each $\Psi \in \mathcal{T}_{\mathcal{C}}$, $q_{1}, q_{2} \in \operatorname{Per}(\Psi)$.

Proof. Using the calculations in Section 5.4, we see, that the last $g$ columns of (5.4.2) are integer iff (5.5.2) holds for $q_{1}$ and for $q_{2}$. Therefore, by Theorem 5.8, there exists a real $g$-parameter family $\mathcal{S}_{\mathcal{C}}$ of CMC-immersions, such that $q_{1}, q_{2} \in \operatorname{Sym}(\Psi)=\operatorname{Sym}\left(\Psi_{\lambda}\right)$ for all $\Psi \in \mathcal{S}_{\mathcal{C}}$ and all $\lambda \in S^{1}$. Since all 
elements of the associated family of a CMC-immersion have the same symmetry group, there is at least a $g-1$-parameter family of associated families $\left\{\Psi_{\lambda} ; \lambda \in S^{1}\right\}$, such that $q_{1}$ and $q_{2}$ are in $\operatorname{Sym}\left(\Psi_{\lambda}\right)$ for all $\lambda \in S^{1}$. From Corollary 5.8 we get that the first column of (5.4.2) is integer iff Condition 2. of Corollary 5.8 is satisfied. By (5.2.17), we have $\Omega_{1}\left(P_{2}\right)=-\Omega_{1}\left(P_{1}\right)$. Therefore, $\Omega_{1}$ vanishes at $P_{1}$ iff it vanishes at $P_{2}$. Now we define $\omega_{1}$ and $\omega_{2}$ for $q_{1}$ and $q_{2}$ by (5.2.16). If $\Omega_{1}\left(P_{1}\right)=0$, then also, by (5.2.16), $\omega_{1}\left(P_{1}\right)=\omega_{2}\left(P_{1}\right)=0$. This shows, that for both, $q_{1}$ and $q_{2}$, the conditions in Corollary 5.8 are satisfied, i.e., $q_{1}, q_{2} \in \operatorname{Per}\left(\Psi_{\lambda_{0}}\right)$. I.e., there exists a $g-1$-parameter family $\mathcal{T}_{\mathcal{C}}=\left\{\Psi_{\lambda_{0}} ; \Psi=\Psi_{1} \in \mathcal{S}_{\mathcal{C}}\right\}$.

In [9], existence of such families of CMC-tori was proved for arbitrary even genus $g>1$. The result was extended to Riemann surfaces of odd genus in $[15]$.

Remark. 1 . In the case $g=1$, the differential $\Omega_{1}$ can be easily computed explicitly: Since $\Omega_{1}$ has a pole of second order at $P_{\infty}$, we know $\Omega_{1}=\frac{a \nu+b}{\mu} \mathrm{d} \nu$. Writing this in the coordinate $\lambda$ and comparing with (5.2.12), we see $a=\frac{1}{2}$. The function $b$ will be determined from the condition (5.2.13). For this we represent the cycle $a_{1}$ by the straight line connecting the points $\nu_{1}=r e^{i \phi}$ and $\nu_{2}=r^{-1} e^{i \phi}$. Then it is straightforward to compute

$$
\int_{a_{1}} \Omega_{1}=e^{i \frac{\phi}{2}} \frac{2 i}{\sqrt{r}} E\left(\sqrt{1-r^{2}}\right)+4 i b \sqrt{r} e^{-i \frac{\phi}{2}} K\left(\sqrt{1-r^{2}}\right),
$$

where $K$ and $E$ denote the complete elliptic differentials of the first and second kind (see e.g. [12]). Now $\int_{a_{1}} \Omega_{1}=0$ yields

$$
b=-\frac{e^{i \phi}}{2 r} \frac{E\left(\sqrt{1-r^{2}}\right)}{K\left(\sqrt{1-r^{2}}\right)} .
$$

If our choices would lead to a torus, then by Theorem 5.4 we would need to have $\Omega_{1}\left(\lambda_{0}\right)=0$ for some point $\lambda_{0} \in S^{1}$. But this equivalent with $|b|=\frac{1}{2}$ which, in view of our formulas, is equivalent with

$$
\frac{E\left(\sqrt{1-r^{2}}\right)}{r K\left(\sqrt{1-r^{2}}\right)}=1 .
$$

But the left hand side is always $>1$ for $0<r<1$, producing a contradiction.

2 . If we only want to produce Delaunay surfaces from hyperelliptic curves of genus 1 , then we start as above and consider $\Omega_{1}$. The coefficients $a$ and $b$ are determined as above. However, we do not need to require $\Omega_{1}\left(P_{1}\left(\lambda_{0}\right)\right)=0$ for some $\lambda_{0} \in S^{1}$ as above, but only (5.9.1). Since $g=1$, this is only one 
equation for $U_{1}$. Obviously, this equation is solvable. Thus in the case $g=1$ our construction produces a family of Delaunay surfaces, indexed by $0<r<1$ and the scale factor of $a^{2}$ in our construction. For a thorough investigation of the case $g=1$ see [20, Chapter 8].

3. It is well known, that there are only two one-parameter families of Delaunay surfaces, the unduloids and the nodoids [8]. Thus, the discussion of Delaunay surfaces above also shows, that the $g$-parameter families constructed above can contain many congruent surfaces. It would certainly be interesting to further investigate this question.

5.11. In Section 5 we have reproduced the algebro-geometric description of CMC-tori $[1,20]$ using the $r$-dressing method. It is important to note, that our starting point differs from the one in [20] and [1].

Pinkall and Sterling started with the observation, that the metric of a CMC-torus is a finite type solution of the sinh-Gordon equation. Thus, as follows from the Krichever construction of finite type solutions to integrable systems, there is a hyperelliptic curve associated to each CMC-torus. However, the analytic construction of this surface from a finite type solution is not unique. Actually, for each trivial flow of a given finite type solution, there is a, possibly singular, hyperelliptic curve. The ambiguity of the definition of the hyperelliptic curve introduces an additional level of complication in the analytic description. While Pinkall and Sterling restricted their attention to nonsingular hyperelliptic curves, Bobenko [1, Appendix] gave an argument why CMC-tori belong generically to nonsingular curves.

Our approach is more geometric in the sense, that we start directly from the periodicity conditions of the extended frame of a CMC-immersion. In Section 4, we introduced a hyperelliptic curve $\mathcal{C}$, which is directly derived from the periodicity conditions on the extended frame of a CMC-immersion with periodic metric. By Theorem 4.9, if the metric of a CMC-immersion in the $r$-drssing orbit of the standard cylinder is periodic, then it is of finite type, and our hyperelliptic curve $\mathcal{C}$ coincides with one of those used in the Krichever construction. However, by definition, the hyperelliptic curve chosen in this paper is always nonsingular. We thereby circumvent the problem of dealing with singular curves.

Of course, there still remains the question, if the construction in Sections 5.5-5.8 gives all CMC-tori.

Let $\Psi: \mathbb{C} \rightarrow \mathbb{R}^{3}$ be a CMC-torus. Then, by [7, Corollary 5.3], $\Psi$ is in the $r$-dressing orbit of the standard cylinder for some $0<r<1$. Hence, by Theorem 3.7, there are rational functions $a^{2}, b^{2}$ and $c^{2}$, satisfying certain conditions, such that $\Psi$ is obtained by $r$-dressing with $h_{+} \in \Lambda_{r} \mathbf{S L}(2, \mathbb{C})_{\sigma}$, 
where $h_{+} A h_{+}^{-1}=\left(\begin{array}{cc}a & b \\ c & -a\end{array}\right)$. Moreover, by Theorem 5.4, there is a nonsingular hyperelliptic curve $\mathcal{C}$ such that $a^{2}$ is the square of a meromorphic function on $\mathcal{C}$.

Conversely, if we start from a hyperelliptic curve $\mathcal{C}$ as in Section 5.5, then the construction in Section 5.7 and Section 5.8 gives only functions $a^{2}, b^{2}$ and $c^{2}$ with simple poles. It is easy to check, that the construction gives in fact all rational functions $a^{2}, b^{2}, c^{2}$ with only simple poles, which have the properties stated in Theorem 3.7.

The question, if this is enough to get all CMC-tori, leads to a statement analogous to the one made in the appendix of [1]:

Conjecture. Let $\Psi: \mathbb{C} \rightarrow \mathbb{R}^{3}$ be a CMC-immersion, which is obtained by dressing the cylinder with $h_{+} \in \Lambda_{r}^{+} \mathbf{S L}(2, \mathbb{C})_{\sigma}$ for some $0<r<1$. Define $h_{+} A h_{+}^{-1}=\left(\begin{array}{cc}a & b \\ c-a\end{array}\right)$. If $\Psi(\mathbb{C})$ is a CMC-torus, then the function $a^{2}(\lambda)$ is rational and has generically only poles of first order.

We would think that this is likely to be true. In this case, generically, to each hyperelliptic curve $\mathcal{C}$ there would exist either no CMC-torus or, up to finitely many choices, precisely the $g-1$-parameter family of CMC-tori $\mathcal{T}_{\mathcal{C}}$ constructed in Theorem 5.10.

We would expect that the understanding of singular tori - if they existwill provide the technical tools to answer these questions.

\section{References}

[1] A. Bobenko, All constant mean curvature tori in $R^{3}, S^{3}, H^{3}$ in terms of thetafunctions, Math. Ann., 290 (1991), 209-245.

[2] F. Burstall and F. Pedit, Dressing orbits of harmonic maps, Duke Math. J., 80 (1995), 353-382.

[3] J. Dorfmeister and G. Haak, Meromorphic potentials and smooth surfaces of constant mean curvature, Math. Z., 1996, to appear.

[4] - On symmetries of constant mean curvature surfaces, Sfb 288 preprint 197, 1996.

[5] J. Dorfmeister, I. McIntosh, F. Pedit and H. Wu, On the meromorphic potential for a harmonic surface in a k-symmetric space, Math. Z., 1996, to appear.

[6] J. Dorfmeister, F. Pedit and H. Wu, Weierstraß type representations of harmonic maps into symmetric spaces, preprint KITCS94-4-1, GANG and KITCS, 1994.

[7] J. Dorfmeister and H. Wu, Constant mean curvature surfaces and loop groups, J. reine Angew. Math., 440 (1993), 43-76.

[8] J. Eells, The surfaces of Delaunay, Math. Intelligencer, 9 (1987), 53-57. 
[9] N.M. Ercolani, H. Knörrer and E. Trubowitz, Hyperelliptic curves that generate constant mean curvature tori in $R^{3}$, in 'Integrable systems' (Luminy, 1991), Progr. Math., 115, Birkhäuser, Boston, (1993), 81-114.

[10] H. Farkas and I. Kra, Riemann surfaces, Springer, Berlin, Heidelberg, New York, 1991.

[11] O. Forster, Riemannsche Flächen, Springer, Berlin, Heidelberg, New York, 1977.

[12] I. Gradshteyn and I. Ryshik, Tables of integrals, series and products, Academic Press, New York, 1965.

[13] G. Haak, Analytische Untersuchungen an klassischen und quantenintegrablen Systemen, Thesis, FU-Berlin, 1993.

[14] G. Haak, M. Schmidt and R. Schrader, Group theoretic formulation of the SegalWilson approach to integrable systems with applications, Rev. Math. Phys., 4 (1992), 451-499.

[15] C. Jaggy, On the classification of constant mean curvature tori in $R^{3}$, Comment. Math. Helv., 69 (1994), 640-658.

[16] N. Kapouleas, Constant mean curvature surfaces constructed by fusing Wente tori, preprint.

[17] Compact constant mean curvature surfaces in Euclidean three-space, J. Diff. Geom., 33 (1991), 683-715.

[18] I. McIntosh, Global solutions of the elliptic 2D periodic Toda lattice, Nonlinearity, 7 (1994), 85-108.

[19] R. Miranda, Algebraic curves and Riemann surfaces, Graduate Studies in Mathematics, Vol. 5, American Mathematical Society, 1995.

[20] U. Pinkall and I. Sterling, On the classification of constant mean curvature tori, Annals of Math., 130 (1989), 407-451.

[21] G. Segal and G. Wilson, Loop groups and equations of KdV type, Publ. Math. I.H.E.S., 61 (1985), 5-65.

Received September 20, 1996 and revised February 19, 1997. The second author was supported by Sonderforschungsbereich 288 .

UNIVERSITY OF KANSAS

LAWRENCE, KS 66045

E-mail address: dorfmeister@ukanvax.bitnet 